\title{
Impact of parameterized lee wave drag on the energy budget of an eddying global ocean model
}

\author{
David S. Trossman ${ }^{\mathrm{a}, *}$, Brian K. Arbic ${ }^{\mathrm{a}}$, Stephen T. Garner ${ }^{\mathrm{b}}$, John A. Goff ${ }^{\mathrm{c}}$, Steven R. Jayne ${ }^{\mathrm{d}}$, \\ E. Joseph Metzger ${ }^{\mathrm{e}}$, Alan J. Wallcraft ${ }^{\mathrm{e}}$ \\ ${ }^{a}$ Department of Earth and Environmental Sciences, University of Michigan, Ann Arbor, MI 48109-1005, USA \\ ${ }^{\mathrm{b}}$ NOAA/Geophysical Fluid Dynamics Laboratory, Princeton, NJ 08542, USA \\ ' Institute for Geophysics, Jackson School of Geosciences, University of Texas, Austin, TX 78758, USA \\ d Physical Oceanography Department, Woods Hole Oceanographic Institution, Woods Hole, MA 02543, USA \\ e Oceanography Division, Naval Research Laboratory (NRL-SSC), Stennis Space Center, MI 39529, USA
}

\section{A R T I C L E I N F O}

\section{Article history:}

Received 13 February 2013

Received in revised form 24 July 2013

Accepted 15 August 2013

Available online 26 August 2013

\section{Keywords:}

Drag

Lee wave

Energy budget

Momentum equations

Viscosity

Abyssal hills

Wind power input

\begin{abstract}
A B S T R A C T
The impact of parameterized topographic internal lee wave drag on the input and output terms in the total mechanical energy budget of a hybrid coordinate high-resolution global ocean general circulation model forced by winds and air-sea buoyancy fluxes is examined here. Wave drag, which parameterizes the generation of internal lee waves arising from geostrophic flow impinging upon rough topography, is included in the prognostic model, ensuring that abyssal currents and stratification in the model are affected by the wave drag.

An inline mechanical (kinetic plus gravitational potential) energy budget including four dissipative terms (parameterized topographic internal lee wave drag, quadratic bottom boundary layer drag, vertical eddy viscosity, and horizontal eddy viscosity) demonstrates that wave drag dissipates less energy in the model than a diagnostic (offline) estimate would suggest, due to reductions in both the abyssal currents and stratification. The equator experiences the largest reduction in energy dissipation associated with wave drag in inline versus offline estimates. Quadratic bottom drag is the energy sink most affected globally by the presence of wave drag in the model; other energy sinks are substantially affected locally, but not in their global integrals. It is suggested that wave drag cannot be mimicked by artificially increasing the quadratic bottom drag because the energy dissipation rates associated with bottom drag are not spatially correlated with those associated with wave drag where the latter are small. Additionally, in contrast to bottom drag, wave drag is a non-local energy sink.

All four aforementioned dissipative terms contribute substantially to the total energy dissipation rate of about one terawatt. The partial time derivative of potential energy (non-zero since the isopycnal depths have a long adjustment time), the surface advective fluxes of potential energy, the rate of change of potential energy due to diffusive mass fluxes, and the conversion between internal energy and potential energy also play a non-negligible role in the total mechanical energy budget. Reasons for the $<10 \%$ total mechanical energy budget imbalance are discussed.
\end{abstract}

(c) 2013 Elsevier Ltd. All rights reserved.

\section{Introduction}

In this paper, we investigate the impact of parameterized topographic internal lee wave drag on the input and output terms in the total mechanical energy budget of a high-resolution ("eddying") global ocean model. We are motivated by the potentially important role of topographic internal lee wave drag in mixing the abyssal ocean. In recent years, there has been great interest in the ocean energy budget, largely because the mixing associated with energy

\footnotetext{
* Corresponding author. Tel.: +1 2065504365.

E-mail address: dtross@umich.edu (D.S. Trossman).
}

dissipation is thought to exert an important control on the largescale circulation. Munk and Wunsch (1998) and St. Laurent and Simmons (2006) have suggested that about 2-3 TW of mixing energy is required to raise diffusivity enough to maintain the abyssal stratification in the presence of the 30 Sverdrups $\left(S v=10^{6} \mathrm{~m}^{3} \mathrm{~s}^{-1}\right)$ of deep water formation. However, Webb and Suginohara (2001) have suggested that maintaining $9 \mathrm{~Sv}$ of Ekman suction in the Southern Ocean while vertically mixing $17 \mathrm{~Sv}$ of North Atlantic Deep Water would reduce the required energy dissipation rate in the abyssal ocean to as little as $0.6 \mathrm{TW}$.

Recently, intense research interest has focused on the sources and sinks of mixing energy in the ocean. Almost all of the 60-68 
terrawatts (TW) of wind power put into the surface waves is dissipated near the surface (Wang and Huang, 2004; Ferrari and Wunsch, 2010) and most of this wind power input is thought to enhance vertical shear of the mean currents (Large et al., 1994) and vertical mixing (Wang et al., 2010; Shu et al., 2011; Qiao and Huang, 2012) in the upper ocean. About 0.4 TW of wind power is put into near-inertial motions in the mixed layer (Watanabe and Hibiya, 1239; Alford, 2003; Furuichi et al., 2008). Early estimates (Wunsch, 1998; Scott and Xu, 2009) of the wind power put into the ocean general circulation, which includes low-frequency currents and mesoscale eddies, were found to be about 0.9 TW. However, Zhai et al. (2012) suggest that only about 0.5 TW of wind power is put into the general circulation because higher frequency wind variability generates shear, mixing, and near-inertial waves in the surface layer rather than deep ocean mixing.

The energy sinks of the low-frequency eddying oceanic general circulation are just beginning to be estimated on a global scale. Topographic internal lee wave drag is a potentially critical component of the mechanical energy budget. Naveira-Garabato et al. (2004), Marshall and Naveira-Garabato (2008), and Nikurashin (2008) suggested that energy is transferred to internal lee waves when geostrophic flow impinges upon rough topographic features and is eventually dissipated, especially in the Southern Ocean where geostrophic flows are strong and the bottom is rough. This energy dissipation mechanism, which is the main focus of the present study, will be referred to simply as "wave drag" hereafter.

Other postulated energy dissipation mechanisms for the eddying general circulation include quadratic bottom boundary layer drag (hereafter, "bottom drag"; Sen et al., 2008; Arbic et al., 2009; and references therein); energy scattering into high-wavenumber vertical modes (Zhai et al., 2010; Saenko et al., 2012); and catalyzed energy exchanges via inviscid balanced flow-boundary interaction (Dewar and Hogg, 2010; Dewar et al., 2011). In ocean models, energy is also dissipated by the vertical eddy viscosity (Large et al., 1994) and horizontal eddy viscosity (Smagorinsky, 1993) that must be employed to make up for the lack of resolved small-scale processes. Vertical eddy viscosity ("vertical viscosity" hereafter) represents processes associated with vertical shear instabilities. Horizontal eddy viscosity ("horizontal viscosity" hereafter) is meant to represent processes that can remove vorticity and momentum at the boundaries of ocean basins (Fox-Kemper and Pedlosky, 2004). Arguably, horizontal viscosity also very roughly represents small-scale processes - for instance, energy transfer from mesoscale eddies to either internal waves (Polzin, 2008) or submesoscale eddies (Müller et al., 2005) - which are not explicitly resolved by any existing numerical global ocean model.

Here, we quantify the relative amounts of energy dissipation of low-frequency flow in an eddying ocean model due to bottom drag, wave drag, horizontal viscosity, and vertical viscosity. Previous estimates suggest that bottom drag and wave drag both contribute substantially to the energy budget of low-frequency flows. For example, Sen et al. (2008) $)^{1}$, Wright et al. (2012), ${ }^{2}$ and Arbic et al. $(2009)^{3}$ have argued that bottom drag dissipates at least 0.2 TW of low-frequency mechanical energy. Arbic and Flierl (2004) and Wright et al. (2013) further argued that some of the energy dissipation that is typically attributed to bottom drag in both models and observations should actually be attributed to wave drag. Nikurashin and Ferrari (2011) estimated the rate of energy dissipation by breaking lee waves to be about 0.2 TW by assuming that this rate is a frac-

\footnotetext{
1 They made use of the Deep Water Archive and Buoy Group Archive. (http:// cmdac.oce.orst.edu/cds.html or http://cmrecords.net)

2 They made use of the more extensive Global Multi-Archive Current Meter Database (http://stockage.univ-brest.fr/scott/GMACMD/updates.html).

3 They utilized multiple eddying ocean models.
}

tion of the energy conversion rate into internal lee waves. In contrast, Scott et al. (2011) estimated the rate of energy conversion into internal lee waves to be about $0.34-0.49 \mathrm{TW}$. Both the Nikurashin and Ferrari (2011) and Scott et al. (2011) estimates are based on bottom stratification fields taken from observations in concert with bottom flows in global ocean models which did not utilize wave drag.

A key contribution of the present manuscript is the prognostic calculation of wave drag and an evaluation of the alterations in kinetic energy, stratification, and sources and sinks of the total mechanical energy budget due to wave drag implementation. Our insertion of wave drag into a global ocean general circulation model is motivated, in part, by demonstrations (e.g., Jayne and St. Laurent, 2001), that the energy budget and accuracy of global forward tide models are impacted to first order by wave drag. Jayne and St. Laurent (2001) found that roughness sufficient to generate internal lee waves occurs quite commonly in the open ocean. Because bottom drag only depends on the bottom velocities and not the roughness, a model simulation that includes a wave drag parameterization should have more dissipation in open ocean regions than a model simulation that only includes a bottom drag parameterization. In addition, there is ample evidence from both observations (Polzin et al., 1997; Naveira-Garabato et al., 2004; St. Laurent et al., 2012) and very high-resolution numerical ocean process models that include bottom drag and wave drag (Nikurashin et al., 2013) that turbulent mixing is enhanced when low-frequency flows encounter rough topography.

While global ocean general circulation models tend to be deficient in bottom kinetic energy relative to current mooring observations (Scott et al., 2011), the correlation between vertical profiles of kinetic energy in ocean models versus observations may be a more important statistic to improve. This is because each ocean model grid point represents an average over a large area, thus tending to smooth the kinetic energy at each model grid point relative to the points at which current meter measurements are taken. Ocean models' simulated kinetic energy increases with finer model resolutions (Thoppil et al., 2011). Therefore, in ocean model simulations without wave drag, bottom kinetic energy may be closer to that of current mooring observations than ocean model simulations with wave drag for the wrong reasons (i.e., inadequate resolution in combination with a lack of abyssal drag such as wave drag). It will be left to a future manuscript to discuss whether the correlation between the kinetic energy profiles in ocean models versus current meter observations is improved with the addition of wave drag.

In this paper, we analyze the global total mechanical energy budget of the total (mean plus eddy) flow, using global nominally $1 / 12^{\circ}$ simulations of the HYbrid Coordinate Ocean Model (HYCOM; http://www.hycom.org; Bleck, 2002; Chassignet et al., 2003; Halliwell, 2004) with and without wave drag. We will not analyze the following: (1) the eddy kinetic energy budget, as was done by Treguier (1992) using a $1 / 3^{\circ} \times 2 / 5^{\circ}$ ocean model of the North Atlantic; (2) the generation and conversion rates between gravitational potential energy and kinetic energy, as was done by Oort et al. (1994) using observations; (3) the mean kinetic energy and gravitational potential energy, as was done by Aiki et al. (2011) using a $1 / 10^{\circ}$ global ocean model; (4) the Lorenz oceanic energy cycle, as was done by von Storch et al. (2012) using a $1 / 10^{\circ}$ global ocean model; or (5) the kinetic plus available potential energy budget, as was done by Hogg et al. (2013) using an idealized $1 / 4^{\circ}$ ocean model that mimicked the Atlantic Ocean. The conversion of kinetic energy to potential energy involves work done by several processes, some of which include horizontal pressure gradients, vertical velocities that result from the convergence or divergence of both the barotropic and baroclinic components of the horizontal velocities, and Reynolds stresses that are mediated by eddy kinetic 
energy (Aiki et al., 2011). The generation of mean kinetic plus potential energy is approximately balanced by the energy dissipators, and the baroclinic energy conversion pathway is facilitated by the conversion of mean kinetic energy to mean potential energy with the surface buoyancy fluxes and winds (von Storch et al., 2012). Here, we look at the kinetic plus gravitational potential (i.e., mechanical) energy budget.

This manuscript is organized as follows. First, we describe the two HYCOM simulations (with versus without wave drag). Then we provide a brief description of the quadratic bottom boundary layer drag parameterization. Next, we introduce the abyssal hill rough topography spectra of Goff and Jordan (1988), Goff and Arbic (2010), and Goff (2010), which are used in the wave drag schemes. We then describe two different wave drag schemes utilized in this paper. Our analysis with the Bell (1975, B75 hereafter) wave drag scheme uses the bottom densities, stratification, and velocities relative to wavevectors in the topographic field's spectral domain to infer an energy conversion rate into lee waves (assumed here to be the energy dissipation rate associated with wave drag). Our analysis with the Garner (2005; G05 hereafter) wave drag scheme uses the stratification, velocities, and features of the underlying topography. Because the G05 scheme can be implemented in the physical domain, it is faster and easier to use inline with a global ocean model. After describing the wave drag schemes and their required input parameters, we perform offline estimates of energy dissipation rates associated with wave drag, as in Nikurashin and Ferrari (2011) and Scott et al. (2011). The offline estimates are useful because they are much less time-consuming to compute than inline estimates. The offline estimates provide a feasible testing ground for comparing the 605 and B75 schemes, and for assessing the impact of using bottom stratification fields taken from HYCOM as opposed to observations. We then run a HYCOM simulation with the G05 scheme inserted, to find inline estimates of the total mechanical energy budget derived here, including energy dissipation rates due to wave drag, bottom drag, vertical viscosity, and horizontal viscosity. We will compare the relative magnitudes of the total mechanical energy budget terms, and we will evaluate how well they balance.

\section{Model description}

The $1 / 12^{\circ}$ HYCOM simulations are on a global tripolar Mercator grid (Murray, 1996) and have 32 hybrid layers in the vertical direction. In the open ocean, the model employs z-level coordinates in the mixed layer and isopycnal coordinates below the mixed layer. In shallow areas, the model employs terrain-following coordinates. This hybrid choice is motivated by the strengths of the different systems in their respective regions (Griffies et al., 2000). Virtual potential density is used, referenced to $2000 \mathrm{~m}$ depth. Unlike potential density, virtual potential density allows the inclusion of buoyancy anomalies caused by thermobaricity by accounting for compressibility when accelerations due to pressure gradients are computed. As described in Sun et al. (1999), the advantages of a virtual potential density include a simple pressure-gradient force expression and monotonicity in depth. Virtual density has the disadvantages of being nonmaterial in adiabatic flow, and of following neutrally buoyant surfaces no better than potential density does. ${ }^{4}$

We now briefly discuss the implementation of eddy viscosities in HYCOM. The K-Profile Parameterization (KPP; Large et al., 1994) is used to determine the vertical viscosity fields, which are derived from the vertical diffusivities with an assumed Prandtl number. For

\footnotetext{
${ }^{4}$ Hallberg (2005) demonstrated that the choice made by Sun et al. (1999) to define virtual potential density can lead to accelerations due to pressure gradients that are numerically unstable in regions with weak stratification.
}

background mixing, which typically is used in deep water, a Prandtl number of three is assumed so that the vertical viscosity is a factor of three larger than the vertical diffusivity. For shear instability mixing, which typically is used in the mixed layer, a Prandtl number of one is assumed. KPP yields relatively strong vertical mixing in the mixed layer, with a smooth transition to weaker vertical mixing below. The horizontal viscosity includes the maximum of a Laplacian and a Smagorinsky (1993) parameterization with an additional biharmonic term. Horizontal viscosity, employed along layers in HYCOM, smoothes out subgrid-scale noise. However, increasing horizontal viscosity generally comes at the expense of model accuracy (Wallcraft et al., 2005; Jochum et al., 2008). Here, "horizontal" means along-isopycnal below the mixed layer in the open ocean, along a constant depth surface within the mixed layer in the open ocean, and parallel to the bathymetry where terrainfollowing coordinates are employed. Referring to the horizontal viscosity term as "lateral viscosity" may be more appropriate, but we refer to this term as horizontal viscosity to be consistent with our reference to vertical viscosity, which may be more accurately called a "diapycnal viscosity". For offline analysis, the viscosity terms, the temperature and salinity fields used to compute the stratification, and the kinetic energy all must be vertically interpolated from hybrid coordinate space to z-levels. Each threedimensional variable in the $k$ th layer $(k=1, \ldots, 32)$ included in the output file from our simulations has been weighted by the thickness of the $k$ th layer at each baroclinic time step and was divided by the average thickness over the present day of the simulation when the output was written to file. The cumulative sums of the thicknesses are the depths, so these are easily interpolated to a regular depth grid, which is the grid we present our zonally averaged results on.

The global model simulations were spun-up from rest using $1.125^{\circ} \times 1.125^{\circ}$ European Centre for Medium-Range Weather Forecasts (ECMWF) Re-Analysis (ERA-40) monthly mean thermal forcing over 1978-2002 (Kallberg et al., 2004). In order to supplement the climatological wind forcing with higher frequencies, six-hourly anomalies with respect to monthly means from the 2003 fields of the Navy Operational Global Atmospheric Prediction System (NOGAPS; Rosmond et al., 2002) was added to the ERA-40 climatological wind forcing. The six-hourly output of 2003 NOGAPS winds were cycled through every model year in this way. In total, HYCOM was spun-up for thirteen years without wave drag. To accelerate the model spin-up period, the assumed background tidal velocity (see, for instance, Willebrand et al., 2001) was not constant throughout the thirteen years. Starting from rest, the background tidal velocity was $5 \mathrm{~cm} \mathrm{~s}^{-1}$ for the first one and onehalf years, $2 \mathrm{~cm} \mathrm{~s}^{-1}$ for the next two and one-half years, and $0 \mathrm{~cm} \mathrm{~s}^{-1}$ for the last nine years. Following the thirteen year spin-up phase without wave drag, an additional simulation was performed for another seven years with an inserted wave drag scheme using an assumed tidal velocity of $0 \mathrm{~cm} \mathrm{~s}^{-1}$. For an offline analysis of wave drag using two different schemes, we used fiveday averaged outputs from one additional (fourteenth) year of the simulation without wave drag.

Our inline total mechanical energy budget analysis was performed on this HYCOM simulation using the final year of the simulation without wave drag (i.e., on the fourteenth year) and using the final year of the simulation with wave drag (i.e., on the twentieth year). We found that results computed from the nineteenth year of the spin-up were nearly equal to results from the twentieth year. This suggests that transient adjustments due to the introduction of wave drag, or to the changing background tidal velocities during spin-up, are generally not large by the time we compute our energy budgets. The globally integrated mean kinetic energy, shown in Fig. 1, demonstrates that the simulation without wave drag is reasonably well spun-up after thirteen years and that the 


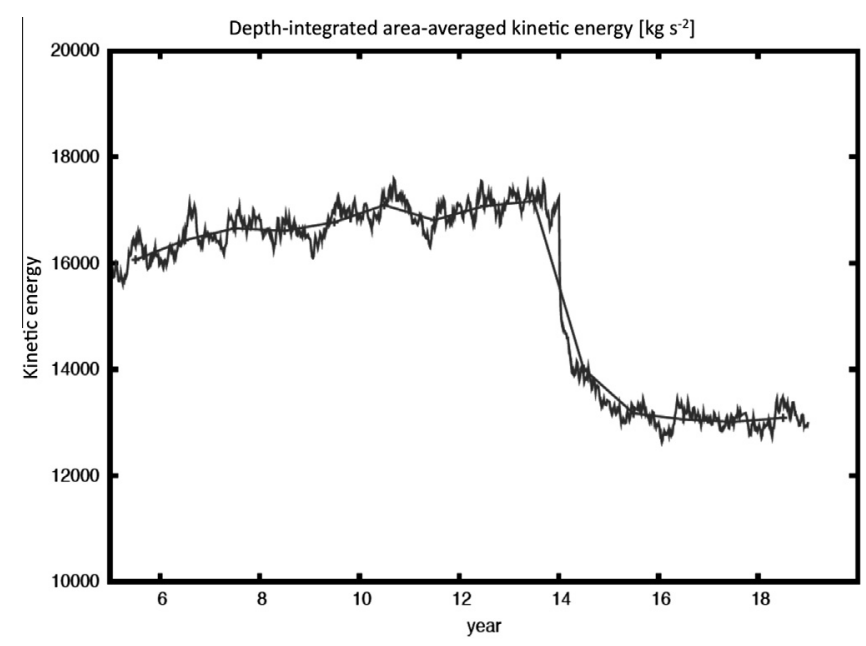

Fig. 1. The global mean (depth-integrated, area-averaged) kinetic energy $\left[\mathrm{kg} \mathrm{s}^{-2}\right]$, $\int d z \int d A\left\{0.5\left(u^{2}+v^{2}\right) \rho\right\} / \int d A$, as a function of spin-up year of the simulation without wave drag (years 5-13) and continuation upon the addition of wave drag (years 14-19). Here, $\rho$ is the density, $\int d z$ indicates an integral over all depths, and $\int d A$ indicates an integral over all horizontal grid locations.

simulation with wave drag is reasonably well spun-up after an additional six years. An additional diagnostic, the area-weighted center of mass of the ocean, also suggests that the model is reasonably well spun-up (not shown). While the globally integrated kinetic energy decreases upon insertion of wave drag, the globally integrated potential energy increases upon insertion of wave drag. We will evaluate the time derivatives of the globally integrated kinetic energy and gravitational potential energy to address how important transient effects are to our total mechanical energy budget.

Observational evidence that wave drag should be applied in regions where there are abyssal hills comes from St. Laurent et al. (2012) and Waterman et al. (2013). A critical decision regarding simulations with wave drag is what to do in non-abyssal hill regions, defined here as the locations where the abyssal hill rough topography spectra of Goff and Jordan (1988) are not defined. Because the abyssal hill rough topography spectra (see Section 3.2) utilized here are strictly valid only for abyssal hill regions, we attempted a model simulation in which wave drag was applied only in abyssal hill regions. However, we found that this simulation was not numerically stable because adjacent grid points attempt to apply drags that are orders of magnitude different from each other. Complementary evidence that the addition of a wave drag field with sharp spatial gradients can lead to numerical problems was found in Arbic et al. (2010), who applied wave drag to tides embedded in an eddying HYCOM simulation. Note the spuriously large velocities in the Gulf of Mexico and other regions in Fig. 2b of Arbic et al. (2010) relative to their Fig. 2a. In subsequent versions of HYCOM simulations with embedded tides (e.g., Shriver et al., 2012), it was found that smoothing the wave drag fields eliminated such artifacts. All of this suggests that applying wave drag in non-abyssal hill regions leads to smoother numerics in the model.

The question then becomes: is it physically justifiable to apply wave drag in regions without abyssal hills? In other words, is roughness in non-abyssal hill regions substantial enough to generate lee waves? Observational justification for applying wave drag in non-abyssal hill regions comes from Kunze et al. (2012) and Beaird et al. (2012). The former study found that the breaking of internal waves on ridges and in canyons, which can be found in non-abyssal hill regions, can be a significant sink of energy (an order of magnitude more energy dissipated locally than in the open ocean) and an enhancer of diffusivity (two to three times higher lo- cally than in the open ocean). A microstructure survey of the Iceland-Faroe Ridge, performed in the latter study, found that the turbulent kinetic energy dissipation was enhanced downstream of the Faroe Bank Channel sills and in the overflow region along the shelf break of Iceland. Neither of these observational studies found that lee waves are necessarily generated in their respective regions, but they do suggest that energy dissipation is enhanced in non-abyssal hill regions with rough topography. Because of the numerical problems we encountered by masking out wave drag in non-abyssal hill regions and the observational evidence that there is enhanced dissipation in non-abyssal hill regions, here we choose to extend the wave drag parameterization into nonabyssal hill regions.

Strictly speaking, our wave drag parameterizations apply only to low-frequency flows. However, time-filtering the velocities in an actively running model is very expensive computationally. Furthermore, applying wave drag to only one portion of the frequency spectrum of velocity can lead to numerical problems (see, e.g., Arbic et al., 2010). Though the numerical problems can be fixed with some effort in topographic smoothing (Shriver et al., 2012), for simplicity, we assume that the abyssal flows in HYCOM are dominated by low-frequency motions. Some justification for this assumption comes from Arbic et al. (2009; see their Section 6), where it is shown that abyssal flows in eddying models that are not forced by tides, as is the case in the present manuscript, are dominated by low frequencies to a much greater extent than are abyssal flows in current meter observations. It is also worth noting that Garner (2005) used his wave drag scheme on the full velocities in atmospheric models, not on a low-passed component to the velocities. In like manner, Nikurashin and Ferrari (2011) and Scott et al. (2011) did not filter their ocean model velocities before applying their wave drag schemes offline. Thus, there is a precedent for applying lee wave drag schemes to flows that include some high-frequency motions. Section 3.3 describes the G05 scheme we implement here for the inline wave drag simulation. Appendix A describes how several parameters for the wave drag parameterization are estimated. Appendix B describes how wave drag is extended to regions beyond those with abyssal hills.

\section{Bottom drag and wave drag schemes}

In this section, we describe the bottom drag scheme used in our model. We then compare two wave drag schemes in an offline analysis. The quadratic bottom boundary layer drag and G05 wave drag schemes are both implemented inline in our model's momentum equations, which will be described in Section 4.1.

\subsection{Quadratic bottom boundary layer drag}

Motivated by results from idealized quasi-geostrophic turbulence models on the importance of either linear (Arbic and Flierl, 2004) or quadratic (Arbic and Scott, 2008) bottom drag to the statistics of mesoscale eddies, Sen et al. (2008) and Arbic et al. (2009) computed an energy dissipation rate due to bottom boundary layer drag of

$E_{B D}=\rho C_{d}\left|\vec{u}_{b}\right|^{3}$.

Here, $\rho$ is the bottom density of seawater, $C_{d}$ is the quadratic drag coefficient (set to 0.0025 in HYCOM), and $\left|\vec{u}_{b}\right|$ is the magnitude of the flow in the bottom layer. Although the dissipation rate is cubic in the bottom current magnitudes, the bottom stress, $\vec{\tau}_{B D}$, in the momentum equations is given by,

$\vec{\tau}_{B D}=-C_{d}\left|\vec{u}_{b}\right| \vec{u}_{b}$,

which is quadratic in the velocity field; hence the convention of referring to $C_{d}$ as the quadratic drag coefficient. 

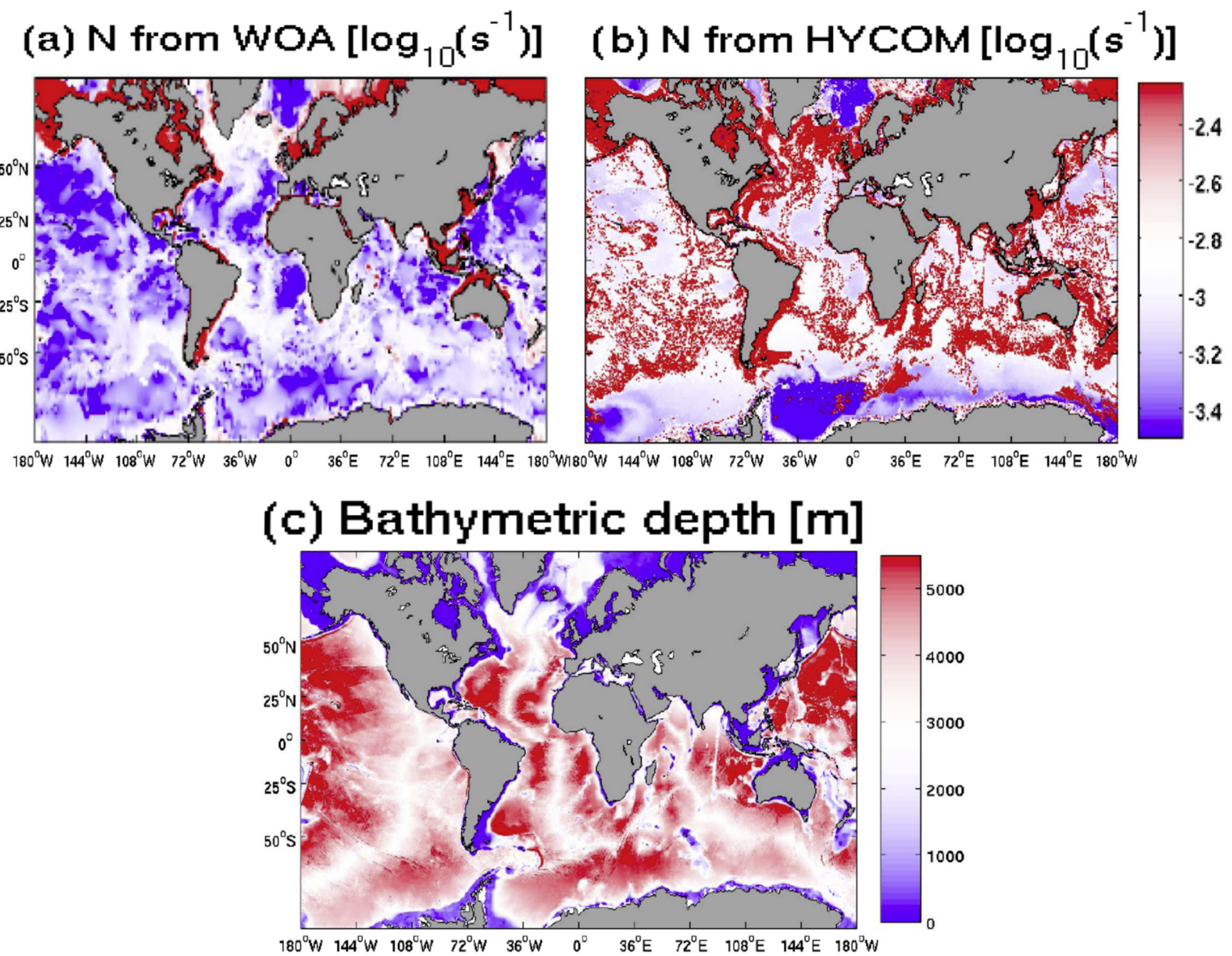

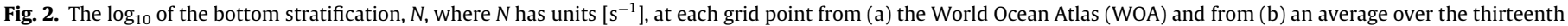
year of the spin-up period from $1 / 12^{\circ}$ HYCOM without wave drag. Also shown is (c) the bathymetic field, which has units [m].

Quadratic bottom boundary layer drag has a long history in oceanography, dating back to Taylor (1919). Evidence that the parameterization in (1) is reasonable comes from Egbert et al. (2004), who demonstrate that the dissipation rates in the shallow seas of a forward global tide model utilizing quadratic drag agree quite well with those inferred from satellite altimetry data. Note that (1) is meant to represent the boundary layer physics in regions with non-negligible velocities and relatively smooth topography. In stratified regions with rough topography, an additional parameterization - one for wave drag - is required. Before delving into the details of the wave drag parameterizations, we first describe one of their key inputs: a statistical representation of the bottom topography in regions where there are abyssal hills.

\subsection{Abyssal hill rough topography representation}

In order to implement wave drag, we require a representation of the bottom topography in the spectral and physical domains. Goff and Jordan (1988) demonstrated the applicability of a twodimensional representation of the von Kàrmàn statistical model for abyssal hill morphology, the primary component of small-scale seafloor roughness. The von Kàrmàn statistical model is a band limited fractal representation, with a power law form at wavenumbers higher than the corner wavenumber, and flat below it. In the anisotropic spectral form proposed by Goff and Jordan (1988), the spectral representation of abyssal hill roughness, $P(k, l)$, is specified by five parameters: root mean square height $(h)$; the power law exponent ( $v$, also identified as the Hurst exponent); corner wavenumbers in the strike and normal-to-strike direction $\left(k_{s}\right.$ and $k_{n}$, respectively); and the azimuth of the strike direction $\left(\xi_{s}\right)$ :

$P(k, l)=4 \pi v h^{2}|\mathbf{Q}|^{-1 / 2}\left(\Upsilon_{*}^{2}(k, l)+1\right)^{-(v+1)}$,

where

$\mathbf{Q}=k_{n}^{2} \hat{\mathbf{e}}_{\mathbf{n}} \hat{\mathbf{e}}_{\mathbf{n}}^{\mathbf{T}}+k_{s}^{2} \hat{\mathbf{e}}_{\mathbf{s}} \hat{\mathbf{e}}_{\mathbf{s}}^{\mathbf{T}}$

(with orthogonal unit vectors, $\hat{\mathbf{e}}_{\mathbf{n}}^{\mathrm{T}} \hat{\mathbf{e}}_{\mathbf{s}}=\mathbf{0}$ ), $\hat{\mathbf{e}}_{\mathbf{n}}$ and $\hat{\mathbf{e}}_{\mathbf{s}}$ are normal to and along the strike directions respectively, and

$\Upsilon_{*}(k, l)=\sqrt{\left(\frac{k}{k_{s}}\right)^{2} \cos ^{2}\left(\xi-\xi_{s}\right)+\left(\frac{k}{k_{n}}\right)^{2} \sin ^{2}\left(\xi-\xi_{s}\right)}$,

where $\xi$ is the azimuth of the wavenumber vector $(k, l)$; hence, the dependence of (5) on both components, $k$ and $l$ (the zonal and meridional directions used in Eqs. (3)-(5)), of the wavenumber vector. Recently, Goff and Arbic (2010) estimated abyssal hill statistical parameters globally using empirical relationships derived previously between spreading rate and direction, as well as the smoothing effects of sediment cover. Goff (2010) then formulated an alternative representation of global abyssal hill statistics based primarily on the small-scale roughness of the gravity field measured 
by satellite altimeters. Although both can be considered realistic renderings, the latter is considered to be more accurate, particularly in more heavily sedimented regions (Goff, 2010). Goff (2010) did not, however, estimate azimuthal orientation. Thus, we will utilize the parameter estimates of Goff (2010) for $h, v, k_{s}$, and $k_{n}$, and of Goff and Arbic (2010) for $\xi_{s}$, to generate the abyssal hill rough topography spectra used for both the B75 and G05 wave drag schemes.

In theory, for the internal lee wave problem, we can synthetically generate a topographic field of arbitrary resolution using (3) to arrive at a roughness input for our wave drag estimates. However, the size of such a synthetic map is prohibitively large. To see this, we employ typical values of the Coriolis parameter, $f \sim 10^{-4} \mathrm{~s}^{-1}$; ocean velocity, $U \sim 10^{-2}$ to $1 \mathrm{~m} \mathrm{~s}^{-1}$; and abyssal Brunt-Väisälä frequency, $N \sim 10^{-3} \mathrm{~s}^{-1}$ in a simple scaling argument for the internal waves to not be evanescent,

$f / U \sim 10^{-4} \mathrm{~m}^{-1}<|\vec{k}|<N / U \sim 10^{-1} \mathrm{~m}^{-1}$.

In (6), we used $U \sim 1 \mathrm{~m} \mathrm{~s}^{-1}$ for the lower bound and $U \sim 10^{-2} \mathrm{~m} \mathrm{~s}^{-1}$ for the upper bound. Examination of the highest wavenumbers arising from (6) suggests that we would need a topographic field of resolution $10 \mathrm{~m}$ or $1 / 10000^{\circ}$ for this task. In order to keep the size of our topographic fields manageable, we seek an alternative formulation (with computations done in spectral space) which does not require such high resolution inputs. Both the B75 and G05 schemes are useful in this sense. It should be noted, however, that $f / U$ and $N / U$ are spatially varying quantities. We cannot know what the stratification or velocity field will be at each horizontal grid point as a function of time before we perform our model simulations. Thus, we need to impose an assumption on the range of relevant wavenumbers via (6).

\subsection{Wave drag schemes}

$B 75$ formulated a scheme that relates a spectral representation of the topographic field's abyssal hill roughness, $P(k, l)$, to the energy flux into lee waves from the geostrophic flow, $\vec{U}$, impinging upon bottom topography. The energy dissipation rate per unit area can be expressed as

$E_{\text {Bell }}=\frac{\rho|\vec{U}|}{2 \pi} \int_{|f| /|\vec{U}|}^{N /|\vec{U}|} d k P(k) \sqrt{\left(N^{2}-|\vec{U}|^{2} k^{2}\right)\left(|\vec{U}|^{2} k^{2}-f^{2}\right)}$,

(B75) where

$P(k)=\frac{1}{2 \pi} \int_{-\infty}^{\infty} d l \frac{|k|}{|\vec{k}|} P(k, l)$,

for $\vec{k}=(k, l)$, the wavenumber vector in the reference frame in which $k$ is along and $l$ is across the mean flow, $\vec{U}$, respectively. The range of wavenumbers used in (7) is determined by (6). This wave drag scheme can be used offline on model output, as in Nikurashin and Ferrari (2011) and Scott et al. (2011). The latter study made use of a multiplicative correction factor, $E_{f a c}$, to the $B 75$ scheme's energy dissipation rate, (7). The correction factor is a function of the Froude number of the bottom flow, $F r=U /(N Z)$, where $Z$ is a vertical length scale:

$E_{f a c}=\frac{L}{\pi}\left[\operatorname{Cos}^{-1}(1-2 L)-2(1-2 L) \sqrt{L(1-L)}\right]$.

Here, $L=1$ when $\mathrm{Fr}^{-1} \leqslant 0.75^{-1}$ and $L=0.75 \mathrm{Fr}$ otherwise. We follow suit in our offline $B 75$ computations, with the intent to compare our results with estimates from Scott et al. (2011). The B75 scheme, and its implementation by Scott et al. (2011), relies on a twodimensional field that represents statistical features of the underlying terrain at each grid point. Furthermore, Scott et al. (2011) only accounts for topographic blocking (i.e., flow that may not propagate over a topographic feature) in an ad hoc way via (9). This motivates an alternative wave drag scheme that we describe below, and that has been successfully used in an altered form (assuming oscillating background flows) in a prognostic ocean tide model (Arbic et al., 2004).

G05 formulated a scheme to parameterize the sink of horizontal momentum due to the interaction of a stratified atmosphere with orography. Using the same scheme, we can parameterize the momentum sink that occurs when low-frequency oceanic flows impinge upon bottom topography. Adding this term to the momentum equations will lead to a sink in the energy as well (see discussion below). In contrast to the B75 scheme, the G05 scheme is based on a calculation of the column-integrated momentum forcing that is exact in the small-amplitude (linear) limit. Since orography is never entirely linear, the G05 scheme adjusts the forcing for partially blocked or partially deflected flow based on a dimensional analysis. An important assumption is that the blocked part of the flow adjusts independently for each topographic feature. This is expected to be less accurate for more densely populated regions of topographic features. ${ }^{5}$ The scheme uses additional assumptions to distribute the forcing in the vertical column. For this preliminary study, we replace these assumptions with a simpler one that all momentum flux is deposited in the lowest $500 \mathrm{~m}$. The implications of a forcing that extends to higher levels are left for a future study.

To calculate the base flux of momentum according to the G05 scheme, we need the information tensor $\mathbf{T}$ (units $\left[\mathrm{kg} \mathrm{m}^{-2} \mathrm{~s}^{-1}\right]$ ), describing the specified subgrid topography. The information tensor can be calculated from

$\mathbf{T}(x, y)=-\left[\begin{array}{l}\frac{\rho N}{(2 \pi)^{2}} \int d k d l|P(k, l)| \frac{k^{2}}{|\vec{k}|} \frac{\rho N}{(2 \pi)^{2}} \int d k d l|P(k, l)| \frac{k l}{|\vec{k}|} \\ \frac{\rho N}{(2 \pi)^{2}} \int d k d l|P(k, l)| \frac{k l}{|\vec{k}|} \frac{\rho N}{(2 \pi)^{2}} \int d k d l|P(k, l)| \frac{l^{2}}{|\vec{k}|}\end{array}\right]=\left[\begin{array}{ll}T_{1,1} & T_{1,2} \\ T_{2,1} & T_{2,2}\end{array}\right]$,

where $\rho$ and $N$ are the instantaneous density and Brunt-Väisälä frequency, respectively, each averaged over the bottom $500 \mathrm{~m}$ (see below). We use the local power spectrum, $P(k, l)$, obtained from (3) using the five parameters estimated from Goff and Arbic (2010) and Goff (2010), to characterize subgrid features of the oceans abyssal hills centered on the grid cell.

Based on the dispersion relation for internal waves, we expect the scales relevant to the wave drag to fall in the range given by (6), which are used to compute T via (10). Scott et al. (2011), by contrast, used the range, $10^{-6}<|\vec{k}|<10^{-1} \mathrm{~m}^{-1}$. However, wavenumbers between $10^{-6} \mathrm{~m}^{-1}$ and $10^{-4} \mathrm{~m}^{-1}$ may only be relevant close to the equator or at mid-latitudes where either $f$ is very small or $U$ is relatively large, making the left-hand side of (6) small. Using the same wavenumber range as Scott et al. (2011) at most doubles the magnitudes of the components of $\mathbf{T}$ in a few scattered locations such as along the Mid-Atlantic Ridge. Because of feedbacks between velocities and wave drag, doubling $\mathbf{T}$ would result in less than a factor of two increase in the dissipation (see, for instance, the $\sim \log _{10}(2)$ reduction with a factor of two increase in drag in Fig. 4a of Arbic et al. (2004) using a forward one-layer ocean tide simulation).

As in G05, we assume that the total drag is in the direction of the linear drag, $\vec{\tau}_{\text {lin }}=\mathbf{T} u_{d}$, and that its magnitude is determined, via the aforementioned dimensional scaling, by

\footnotetext{
5 Another important assumption is that the G05 scheme does not explicitly consider the effects of rotation on the drag itself, the assumption being that $N \gg f$. However, the information tensor used to calculate the wave drag is integrated over the range of wavenumbers specified by (6), which does depend upon the Coriolis parameter.
} 
$\vec{\tau}=\left(\tau_{x}, \tau_{y}\right)=\left(\frac{D_{p}}{D^{*}}+\frac{D_{n p}}{D^{*}}\right)\left(\mathbf{T} \vec{u}_{d}\right)$.

Here, $\vec{u}_{d}$ is the velocity averaged over the bottom $H_{W D}=500 \mathrm{~m}, D_{p}$ and $D_{n p}$ (respectively) are the propagating and non-propagating parts of the drag based on the dimensional analysis, and $D^{*}$ is the linear limit of $D_{p}$. Our choice of $H_{W D}=500 \mathrm{~m}$ is guided by the vertical decay scale in St. Laurent et al. (2002), which is based on the microstructure observations of St. Laurent et al. (2001) in the Brazil Basin. A more recent observational study (St. Laurent et al., 2012) was done in the Drake Passage and found no evidence of enhanced turbulence at heights exceeding $500 \mathrm{~m}$ above the bottom. As in HYCOM simulations with embedded tides (Arbic et al., 2010), $H_{W D}=500 \mathrm{~m}$ is much greater than $H_{B D}=10 \mathrm{~m}$, the thickness over which quadratic bottom drag acts.

The expressions for $D_{p}, D_{n p}$, and $D^{*}$ are taken from $G 05$ without modification - his Eqs. 15,16. For the convenience of the reader, they are given here:

$$
\begin{aligned}
D^{*}= & a_{0} \frac{\rho V^{3}}{N L_{r}} H_{r}^{\gamma}\left[\frac{(2 \gamma-\epsilon)\left(H_{\max }^{2+\gamma-\epsilon}-H_{\min }^{2+\gamma-\epsilon}\right)}{(2+\gamma-\epsilon)\left(H_{\max }^{2 \gamma-\epsilon}-H_{\min }^{2 \gamma-\epsilon}\right)}\right], \\
D_{p}= & a_{0} H_{r}^{\gamma} \frac{\rho V^{3}}{N L_{r}} \frac{2 \gamma-\epsilon}{H_{\max }^{2 \gamma-\epsilon}-H_{\min }^{2 \gamma-\epsilon}} \\
& \times\left(\frac{H_{c l i p}^{2+\gamma-\epsilon}-H_{\min }^{2+\gamma-\epsilon}}{2+\gamma-\epsilon}+H_{\text {crit }}^{2+\beta} \frac{H_{\max }^{\gamma-\epsilon-\beta}-H_{c l i p}^{\gamma-\epsilon-\beta}}{\gamma-\epsilon-\beta}\right), \\
D_{n p}= & \left.a_{1} H_{r}^{\gamma} \frac{\rho V^{3}}{N L_{r}(1+\beta)} \frac{2 \gamma-\epsilon}{H_{\max }^{2 \gamma-\epsilon}-H_{\min }^{2 \gamma-\epsilon}}\right) \\
& \times\left(\frac{H_{\max }^{1+\gamma-\epsilon}-H_{c l i p}^{1+\gamma-\epsilon}}{1+\gamma-\epsilon}-H_{c r i t}^{1+\beta} \frac{H_{\max }^{\gamma-\epsilon-\beta}-H_{c l i p}^{\gamma-\epsilon-\beta}}{\gamma-\epsilon-\beta}\right) .
\end{aligned}
$$

The minimum and maximum terrain heights, $H_{\min }=0.1 H_{\max }$ and $H_{\max }$, are computed in the way suggested by G05 and normalized by $V / N$, the internal scale. For this scale, we use the velocity, $V$, and stratification, $N^{2}$, in the lowest $H_{W D}=500 \mathrm{~m}$ of the model. The terrain height limit that prevents wave drag from increasing beyond a certain extent is $H_{\text {clip }}=\min \left\{H_{\max }, \max \left\{H_{\min }, H_{\text {crit }}\right\}\right\}$, all variables with the subscript ' $r$ ' (for reference) cancel out, and $\widetilde{H}_{c r i t}=N H_{\text {crit }} / V$ is the critical nondimensional height that determines the degree to which the flow is blocked. The critical nondimensional height is a universal parameter in this scheme, leaving $H_{\text {crit }}$ to be flow-dependent. We set $\widetilde{H}_{\text {crit }}=0.7$. Other choices of parameters used in the G05 scheme, such as the coefficients for the propagating and non-propagating components of wave drag ( $a_{0}$ and $a_{1}$, respectively), $\gamma, \epsilon$, and $\beta$ are discussed in Appendix A.

For simplicity, and for a more direct comparison with the B75 scheme, we reduce the parameterized tensor drag in the G05 scheme to a scalar drag with the same instantaneous impact on the kinetic energy. This procedure has been used in tidal simulations (e.g., Arbic et al., 2010). For the stress, $\vec{\tau}$, given in (11) we compute

$r_{\text {drag }}=\frac{\vec{\tau} \cdot \vec{u}_{d}}{\rho\left|\vec{u}_{d}\right|^{2}}$

which is a decay rate times a vertical length scale (units $\left[\mathrm{m} \mathrm{s}^{-1}\right]$ ). The value of $r_{\text {drag }}$ is computed using the full tensor, $\mathbf{T}$, at each grid point inline with HYCOM. To be precise, the stress associated with the wave drag term in the momentum equations is computed as

$\vec{\tau}_{W D}=-\left|r_{\text {drag }}\right| \vec{u}_{d}$.

Thus, in the model momentum equations, $\left|r_{\text {drag }}\right|$ in (14) is to wave drag as $C_{d}\left|\vec{u}_{b}\right|$ in (2) is to bottom drag. While $C_{d}\left|\vec{u}_{b}\right|$ depends only on the velocities in the model's bottom layer, $\left|r_{\text {drag }}\right|$ depends on both
Table 1

The estimated globally integrated energy dissipation rate due to wave drag $\left[\mathrm{TW}=10^{12} \mathrm{~W}\right]$ using two different wave drag schemes: B75 (Bell) and G05 (Garner). We utilize a five-day averaged velocity field, averaged over the bottom $500 \mathrm{~m}$, from the final year of the $1 / 12^{\circ}$ HYCOM spin-up without wave drag. Two different bottom stratification fields are used: from the World Ocean Atlas (WOA) and an average over the bottom $500 \mathrm{~m}$ from the final year of the $1 / 12^{\circ}$ HYCOM spin-up without wave drag.

\begin{tabular}{lll}
\hline Wave drag scheme & WOA & HYCOM \\
\hline Garner & 0.47 & 0.45 \\
Bell & 0.45 & 0.51 \\
\hline
\end{tabular}

the velocities and stratification in the model's bottom $500 \mathrm{~m}$ in addition to features of the underlying topography.

The use of (14) slightly changes the time-dependent flow compared to the full tensor implementation in Arbic et al. (2004) and G05, in which the drag is not constrained to be parallel to the flow. The angle between the flow and drag is mainly determined by the difference between the diagonal elements of $\mathbf{T}$ (the off-diagonal elements are inherently small). In Section 4.2, we will explicitly compare $\vec{\tau} / \rho$ with $r_{\text {drag }} \vec{u}_{d}$.

\section{Energy budgets}

In this section we list the source and sink terms in the total mechanical energy budget. We follow this with a comparison of wave drag contributions to the energy budget using the B75 and G05 schemes acting offline on output from HYCOM simulations that do not have wave drag actively altering the velocities. We also compare offline estimates using bottom stratification fields from both WOA (World Ocean Atlas 2005) ${ }^{6}$ and HYCOM, the latter calculated with the TEOS-10 package (McDougall and Barker, 2011). We compute the input and output terms of an energy budget in line for HYCOM simulations with and without wave drag, the former including the G05 wave drag scheme actively altering both the bottom velocities and bottom stratification fields.

\subsection{Total mechanical energy budget}

The kinetic energy equation, part of the total mechanical energy budget, here is derived from the momentum equations,

$$
\begin{aligned}
\frac{\partial \vec{u}_{H}}{\partial t} & +(\vec{u} \cdot \vec{\nabla}) \vec{u}_{H}+\frac{1}{\rho} \vec{\nabla} p+f \hat{k} \times \vec{u}_{H}+g \hat{k} \\
= & \frac{\theta\left(z>-H_{s}\right)}{\rho} \frac{\vec{\tau}_{\text {wind }}}{H_{s}}-\theta\left(z<H_{B D}-z_{b}\right) \frac{C_{d}}{H_{B D}}\left|\vec{u}_{H}\right| \vec{u}_{H}-\theta(z \\
< & \left.H_{W D}-z_{b}\right) \frac{\left|r_{\text {drag }}\right|}{H_{W D}} \vec{u}_{H}-\frac{\partial}{\partial z}\left(v_{z} \frac{\partial}{\partial z} \vec{u}_{H}\right)-\vec{\nabla} \cdot\left(v_{h, 2} \vec{\nabla} \vec{u}_{H}\right. \\
& \left.+v_{h, 4} \vec{\nabla} \nabla^{2} \vec{u}_{H}\right),
\end{aligned}
$$

where $\vec{\nabla}=(\partial / \partial x, \partial / \partial y, \partial / \partial z)$ is a three-dimensional gradient operator, $\vec{u}_{H}=(u, v)$ is the two-dimensional velocity along isopycnal surfaces, $\vec{u}=(u, v, w)$ is the three-dimensional velocity, $p$ is the pressure, $\hat{k}$ is a unit vector in the vertical direction, $g=9.806 \mathrm{~m} \mathrm{~s}^{-2}$ is the acceleration due to gravity, and $f$ is the Coriolis parameter. The right hand side of (15) displays the wind stress, bottom drag, wave drag, vertical viscosity, and horizontal viscosity terms, respectively. $\theta\left(z<H_{B D}-z_{b}\right)$ is a step function that is one in the layer of thickness $H_{B D}=10 \mathrm{~m}$ from the bottom, and zero for all other layers. $\theta\left(z<H_{W D}-z_{b}\right)$ is a step function that is one in the layer of thickness $H_{W D}=500 \mathrm{~m}$ from the bottom, and zero for all other layers. ${ }^{7}$ $\theta\left(z>-H_{s}\right)$ is a step function that equals one in the surface layer of

\footnotetext{
${ }^{6}$ http://www.nodc.noaa.gov/OC5/WOA05/woa05data.html

7 In regions shallower than $500 \mathrm{~m}, H_{W D}$ is taken to be the depth of the water column.
} 
thickness $H_{s}$ and zero for all other layers. $\vec{\tau}_{\text {wind }}$ is the surface wind stress, which is computed accounting for the relative velocities of the wind and surface currents (Scott and Xu, 2009). The vertical viscosity, $v_{z} \sim 10^{-4}-10^{-3} \mathrm{~m}^{2} \mathrm{~s}^{-1}$, is calculated inline in HYCOM using KPP (Large et al., 1994). The horizontal viscosity, which is on the order of $10^{2}-10^{3} \mathrm{~m}^{2} \mathrm{~s}^{-1}$, is also calculated inline in HYCOM with $v_{h, 2}$ taken to be the maximum of a Laplacian term and a Smagorinsky (1993) term and $v_{h, 4}$ taken to be a biharmonic term.

Multiplying (15) by density, dotting that equation with the velocity, and performing a volume integral, one finds the kinetic energy equation, which includes a conversion term between kinetic energy and potential energy. We first write the kinetic energy equation here for an incompressible, hydrostatic fluid explicitly as

$P_{E_{K} \text { time }}+P_{E_{K} a d v}=P_{\text {pressure }}+P_{\text {input }}-P_{\text {output }}+C_{E_{K}->E_{P}}$

(Griffies, 2004) where the area integrals are evaluated at the surface and arise as a result of invoking Gauss' Theorem, $E_{K}=\rho \vec{u} \cdot \vec{u} / 2$ is the kinetic energy per unit volume, $P_{\text {input }}=P_{\text {wind }}$, $P_{\text {output }}=P_{B D}+P_{W D}+P_{V V}+P_{H V}$, and

$$
\begin{aligned}
& P_{E_{K} \text { time }}=\int d x \int d y\left(\int_{-z_{b}}^{\eta} d z\left[\frac{\rho}{2} \frac{\partial(\vec{u} \cdot \vec{u})}{\partial t}\right]\right), \\
& P_{E_{K} a d v}=\int d x \int d y\left(w E_{K}\right), \\
& P_{\text {pressure }}=-\int d x \int d y(p w), \\
& P_{\text {wind }}=\int d x \int d y\left(\vec{u}_{s} \cdot \vec{\tau}_{\text {wind }}\right), \\
& P_{B D}=\int d x \int d y\left(\rho C_{d}\left|\vec{u}_{b}\right|^{3}\right), \\
& P_{W D}=\int d x \int d y\left(\rho\left|r_{d r a g}\right|\left|\vec{u}_{d}\right|^{2}\right), \\
& P_{V V}=\int d x \int d y\left(\int_{-z_{b}}^{\eta} d z\left[\rho \vec{u} \cdot \frac{\partial}{\partial z}\left(v_{z} \frac{\partial}{\partial z} \vec{u}_{H}\right)\right]\right), \\
& P_{H V}=\int d x \int d y\left(\int_{-z_{b}}^{\eta} d z\left[\rho \vec{u} \cdot \vec{\nabla}\left(v_{h, 2} \vec{\nabla} \vec{u}_{H}+v_{h, 4} \vec{\nabla} \nabla^{2} \vec{u}_{H}\right)\right]\right), \\
& C_{E_{K}->E_{P}}=-\int d x \int d y\left(\int_{-z_{b}}^{\eta} d z[g \rho w]\right) .
\end{aligned}
$$

$P_{E_{K} t i m e}$ denotes the time rate of change in globally integrated kinetic energy, $P_{E_{K} a d v}$ denotes the change in kinetic energy due to advective fluxes across the surface, $P_{\text {pressure }}$ denotes the divergence of kinetic energy due to pressure differentials at the surface (evaluated at the atmospheric pressure $p=p_{a t m}$ which is constant in the model), $C_{E_{K}->E_{P}}$ denotes the conversion of kinetic energy $\left(E_{K}\right)$ to potential energy $\left(E_{P}\right), P_{\text {wind }}$ denotes the wind power input to the total flow, $P_{B D}$ denotes the kinetic energy dissipation due to bottom drag, $P_{W D}$ denotes the kinetic energy dissipation due to wave drag, $P_{V V}$ denotes the kinetic energy dissipation due to vertical viscosity, and $P_{H V}$ denotes the kinetic energy dissipation due to horizontal viscosity, respectively. Here, $\eta$ is the sea surface height, $\int d x \int d y(\cdot)$ indicates an integral over the surface area of the globe and at a given horizontal grid point in HYCOM, $z_{b}$ is the depth of the ocean, $\vec{u}_{b}$ is the two-dimensional velocity averaged over the bottom $H_{B D}$ meters, $\vec{u}_{d}$ is the two-dimensional velocity averaged over the bottom $H_{W D}$ meters, $\vec{u}_{s}$ is the two-dimensional surface velocity, and $w$ is the vertical velocity. In addition to the wind power input to the total flow, we compute the wind power input to the geostrophic flow via $\int d x \int d y\left(\vec{u}_{g} \cdot \vec{\tau}_{\text {wind }}\right)$, where $\vec{u}_{g}$ is the geostrophic velocity derived from the sea surface heights (Wunsch, 1998), in order to evaluate how wave drag impacts the surface in Section 4.3.

It is worth noting that the integrands of both the horizontal and vertical viscosity terms in (17) can represent energy sources (i.e., have positive values) at isolated grid points (see Section 4.3). For horizontal viscosity, this may seem to deviate from the spectral model-based argument that the Laplacian term is a net dissipator. However, the latter argument only applies to an integral over a domain much larger than a single grid point. Fox-Kemper and Menemenlis (2008) note that the Smagorinsky (1993) parameterization takes a domain-averaged value of the horizontal viscosity and replaces it with a local value. Therefore, the small-scale spatial variation in maps of energy dissipation by the horizontal viscosity term should not be interpreted as physical. The small-scale spatial variation of the vertical viscosity term may not have a physical interpretation either. The global integrals and some regional characteristics of the horizontal and vertical viscosity terms in (17), however, are physically meaningful.

The potential energy equation, also part of the total mechanical energy budget, is derived by making use of the equation (Griffies, 2004), $d \rho / d t=\vec{\nabla} \cdot(\kappa \vec{\nabla} \rho)$, where $\kappa$ is the eddy diffusivity tensor; the definition of the vertical velocity, $d z / d t=w$; the potential energy per unit volume, $E_{P}=\rho g z$; the definition of a material derivative,

$\int d x \int d y \int d z \frac{d E_{P}}{d t}=\int d x \int d y \int d z\left[\frac{\partial E_{P}}{\partial t}+\vec{u} \cdot \vec{\nabla} E_{P}\right]$

and Gauss' Theorem,

$$
\begin{aligned}
& \int d x \int d y \int d z \frac{d E_{P}}{d t}=\int d x \int d y \int d z\left[\rho g \frac{d z}{d t}+\frac{d \rho}{d t} g z\right] \\
& =\int d x \int d y \int d z[\rho g w+g z(\vec{\nabla} \cdot(\kappa \vec{\nabla} \rho))]=\int d x \int d y \int d z[\rho g w] \\
& +\int d x \int d y \int d z[g \vec{\nabla} \cdot(\kappa z \vec{\nabla} \rho)]-\int d x \int d y \int d z[g \kappa \hat{k} \cdot \vec{\nabla} \rho] \\
& =\int d x \int d y \int d z[\rho g w]+\int d x \int d y\left[g \eta \kappa \frac{\partial \rho}{\partial z}\right]-\int d x \int d y\left[g z_{b} \kappa \frac{\partial \rho}{\partial z}\right] \\
& +\int d x \int d y \int d z\left[g \vec{\nabla}_{H} \cdot\left(\kappa z \vec{\nabla}_{H} \rho\right)\right]-\int d x \int d y \int d z\left[g \kappa \frac{\partial \rho}{\partial z}\right] .
\end{aligned}
$$

Going from left to right, the terms on the right-hand side of (18) are the time rate of change in potential energy and the reversible changes in potential energy via advective fluxes at the surface. Similarly going from left to right, the terms on the right-hand side (last line) of (19) are the conversion between potential energy and kinetic energy via buoyancy fluxes, the rate of change of potential energy due to diffusive mass flux that acts to mix or stir density (evaluated at the surface and at the seafloor) across isopycnals, the rate of change of potential energy due to diffusive mass flux that acts to mix or stir density along isopycnals, and the conversion from internal energy to potential energy. We assume that the diffusive mass flux (i.e., buoyancy diffusion) evaluated at the seafloor is negligible because there is no geothermal heat flux there in the model. We also neglect the along-isopycnal contributions to the buoyancy diffusion term by assuming that $\vec{\nabla}_{H} \rho=(\partial / \partial x, \partial / \partial y) \rho=\overrightarrow{0}$ in the model, reducing $\kappa$ to a scalar. ${ }^{8}$ We allow for the diffusivity, $\kappa=v_{z}$, at the surface to spatially vary in the diffusive mass flux across isopycnals and conversion from internal energy to potential energy terms on the right-hand side of (19), but (19) is otherwise the same as Eq. (6) in Winters et al. (1995). The vertical velocity at the surface, needed for $P_{E p a d v}$ and $P_{\text {diffusive }}$, is calculated by making use of the surface boundary condition that $w=\partial \eta / \partial t+\vec{u}_{H} \cdot \vec{\nabla} \eta$. We do not include work done associated with the compressibility of fluid here since, by our assumptions, $\vec{\nabla} \cdot \vec{u}=0$. The buoyancy flux, or thermodynamic work, term at the surface does not explicitly appear in the conservation equation because, as argued by Wunsch and Ferrari

\footnotetext{
${ }^{8}$ When $\vec{\nabla}_{H} \rho \neq \overrightarrow{0}$, it is unclear how to compute the along-isopycnal diffusivities as HYCOM runs because the Laplacian and biharmonic contributions to the horizontal viscosity have diffusion velocities from which we can compute diffusivities, but the Smagorinsky term does not.
} 
(2004), the buoyancy flux at the surface enters as internal energy which then can be converted to other forms of energy by changing the mass of the ocean via the last term in (19). Saenz et al. (2012) suggest that the available potential energy ultimately provided by the buoyancy flux at the surface can be converted into kinetic energy via the first term on the right-hand side of (19). Tailleux (2010) suggests that the buoyancy flux term may be a significant contributor to the energy budget, and von Storch et al. (2012) found, using their high-resolution ocean model, that the contribution of the buoyancy flux to the input of potential energy is comparable to the wind power input.

By equating (18) with (19), we write the potential energy equation succinctly here as

$P_{E_{p} \text { time }}+P_{E_{P} a d v}=P_{\text {diffusive }}+C_{E_{P}->E_{K}}+C_{E_{I}->E_{P}}$,

where

$$
\begin{aligned}
& P_{E_{p} \text { time }}=\int d x \int d y\left(\int_{-z_{b}}^{\eta} d z\left[\frac{\partial E_{P}}{\partial t}\right]\right) \\
& P_{E_{p} a d v}=\int d x \int d y(\eta g \rho w) \\
& P_{\text {diffusive }}=\int d x \int d y\left(g \eta \kappa \frac{\partial \rho}{\partial z}\right) \\
& C_{E_{P}->E_{K}}=\int d x \int d y\left(\int_{-z_{b}}^{\eta} d z[g \rho w]\right) \\
& C_{E_{I}->E_{P}}=-\int d x \int d y\left(\int_{-z_{b}}^{\eta} d z\left[g \kappa \frac{\partial \rho}{\partial z}\right]\right) .
\end{aligned}
$$

$P_{E_{p} t i m e}$ denotes the time rate of change in globally integrated potential energy, $P_{E_{p} a d v}$ denotes the changes in potential energy due to advective fluxes at the surface, $P_{\text {diffusive }}$ denotes the potential energy change due to diffusive mass fluxes (buoyancy diffusion), $C_{E_{P}->E_{K}}$ denotes the conversion of potential energy to kinetic energy, and $C_{E_{I}->E_{p}}$ denotes the conversion from internal energy $\left(E_{I}\right)$ to potential energy. The advective flux of potential energy should be thought of as the work the ocean is doing on the atmosphere. Again, note that here we evaluate the diapycnal component of the diffusive mass fluxes. The buoyancy diffusion term, $P_{\text {diffusive }}$, can be interpreted as the power associated with density mixing or stirring. Finally, our total mechanical energy budget is calculated by summing (16) and (20), yielding

$$
\begin{aligned}
& P_{E_{K} \text { time }}+P_{E_{P} \text { time }}+P_{E_{K} a d v}+P_{E_{P} a d v} \\
& \quad=P_{\text {pressure }}+P_{\text {diffusive }}+C_{E_{I^{-}}>E_{P}}+P_{\text {input }}-P_{\text {output }} .
\end{aligned}
$$

Each of the advective flux terms and the pressure term make use of the incompressibility assumption.

It is important to note that, with the exception of the partial time derivative terms, the terms in (22) in our inline calculations are evaluated instantaneously at each baroclinic time step of two minutes. For example, the average of $\vec{u}_{s} \cdot \vec{\tau}_{\text {wind }}$ is computed instead of the product of the averages of $\vec{u}_{s}$ and $\vec{\tau}_{\text {wind }}$. Thus, we are confident that aliasing higher frequency motions forced by 2003 NOGAPS winds is not a problem. The partial time derivative terms, $P_{E_{K} \text { time }}$ and $P_{E_{p} t i m e}$, are diagnosed using the global integrals of monthly averaged fields from the final year of the HYCOM simulation with wave drag.

\subsection{Preliminary offline estimates of wave drag}

In this section, we perform offline estimates of energy dissipation rates due to wave drag. First, we show that the G05 scheme yields spatial maps and globally integrated dissipation rates that are comparable to those computed from the B75 scheme. To do this, we use five-day averaged velocities, averaged over the bottom
$500 \mathrm{~m}$, from the fourteenth year of a $1 / 12^{\circ}$ HYCOM simulation spun-up without wave drag. We use two fields for the mean annual abyssal stratification: one taken from the same model simulation, and the other taken from WOA. The WOA stratification (Fig. 2a) is typically weaker than the HYCOM stratification (Fig. 2b), particularly in areas of rough abyssal hills that flank topographic ridges (Fig. 2c). For the offline estimates, we also use the abyssal hill rough topography power spectra that were computed with parameters from Goff (2010) and Goff and Arbic (2010).

The offline globally integrated energy dissipation rates due to wave drag are listed in Table 1, while spatial maps are shown in Fig. 3. When the WOA bottom stratification field is used, the globally integrated energy dissipation rate using the 605 scheme is larger than that using the $B 75$ scheme by about $5 \%$. On the other hand, the globally integrated energy dissipation rate using the G05 scheme is smaller than those using the $B 75$ scheme by about $10 \%$ when the HYCOM stratification field, averaged over the bottom $500 \mathrm{~m}$, is used. These differences are likely because the B75 scheme does not explicitly distinguish between flow that propagates and flow that does not propagate over a topographic feature, whereas the G05 scheme does. The nonlinearity of the G05 scheme depends on the stratification, and the B75 scheme depends differently upon the stratification. It is also possible that the differences are due to the explicit dependence of the B75 estimates upon the Coriolis parameter. However, the discrepancies do not appear to be related to the Coriolis parameter because differences between the upper panels (G05 scheme) and lower panels (B75 scheme) in Fig. 3 do not appear to be latitudinally dependent any more than they are longitudinally dependent.

The regions with the greatest energy dissipation rates using either wave drag scheme are in the Subpolar North and South Atlantic Oceans, the western Indian Ocean, and the Southern Ocean (Fig. 3). The B75 scheme has larger dissipation rates than the G05 scheme off the coast of Brazil, in the South Pacific Ocean, and in the western Indian Ocean. On the other hand, the B75 scheme has smaller dissipation rates than the G05 scheme in the wake of the Drake Passage, in the Antarctic Circumpolar Current (ACC), in the Aghulas Current region, and in the Gulf Stream Extension region. Both the B75 and G05 schemes estimate greater dissipation rates (Fig. 3 ) in regions where we would expect more energy dissipation due to a combination of relatively strong flow (not shown) and relatively strong stratification (Fig. 2 b) over rough topographic features (Fig. 2c).

Our offline dissipation rates using the WOA stratification are larger than those of Nikurashin and Ferrari (2011) (0.2 TW) and are closer to those of Scott et al. (2011) (0.34-0.49 TW). We have used a similar WOA stratification field and the same abyssal hill rough topography power spectra as the larger of the estimates of Scott et al. (2011), but our abyssal velocity field is different. The abyssal stratification field, abyssal velocity field, and abyssal hill rough topography power spectrum formulation are all different here from those used in Nikurashin and Ferrari (2011). Nikurashin and Ferrari (2011) used an isotropic spectral form of the abyssal hills, while Scott et al. (2011) allowed for anisotropy to enter into the abyssal hill power spectra. The largest difference between the estimates of Nikurashin and Ferrari (2011) versus those in Scott et al. (2011) and in the present study is along the equator, where the former study found much larger dissipation rates. Because each of the aforementioned studies used similar stratification products, it is likely that the relatively large dissipation rates found along the equator in the estimates of Nikurashin and Ferrari (2011) are due to their use of an isotropic spectral form of the abyssal hill power spectrum. However, the velocity field used by Nikurashin and Ferrari (2011) could be responsible for their relatively large dissipation rates found along the equator. The stratification field may also be important for a given velocity field, evidenced by the 
(a) (Garner scheme, N from WOA)

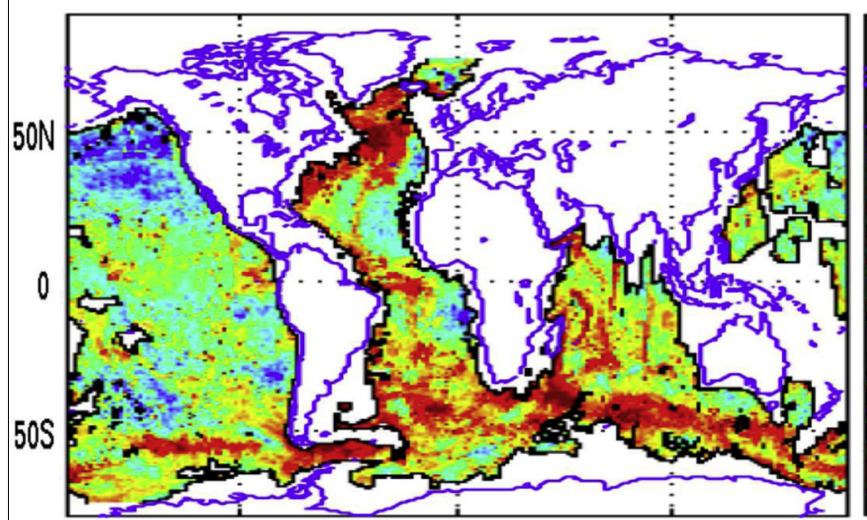

(c) (Bell scheme, N from WOA)

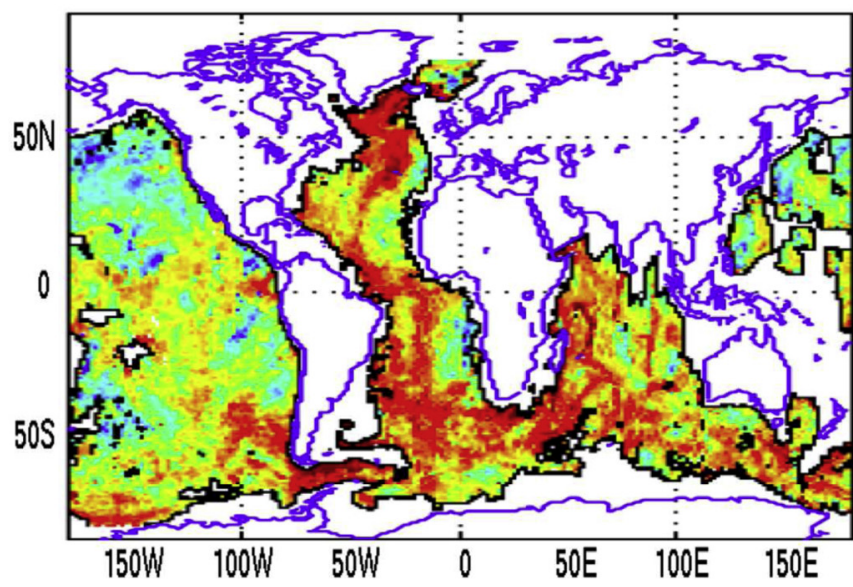

(b) (Garner scheme, N from HYCOM)

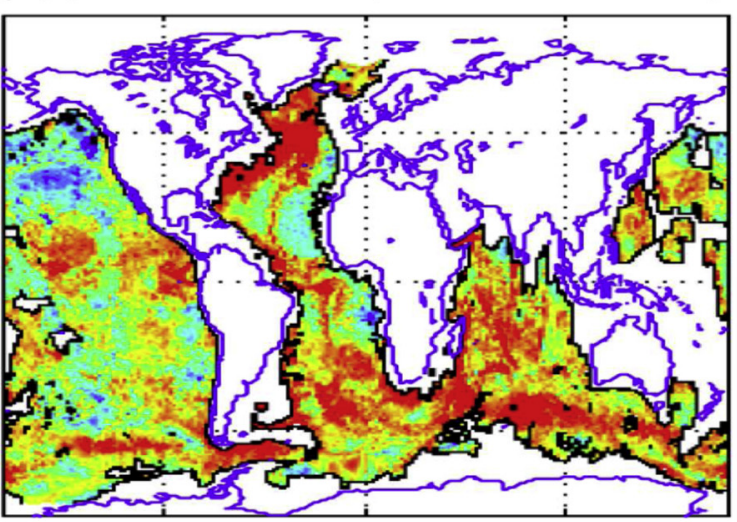

(d) (Bell scheme, $\mathrm{N}$ from HYCOM)

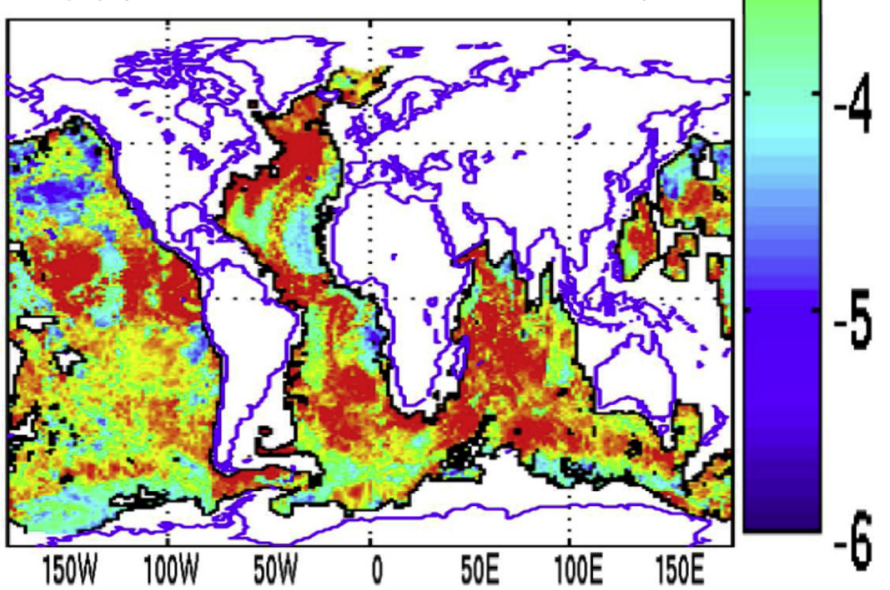

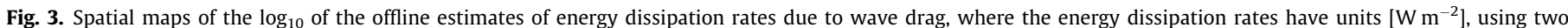

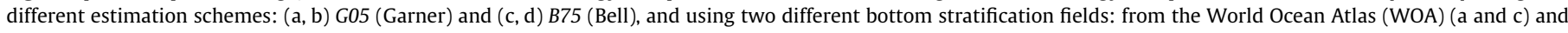

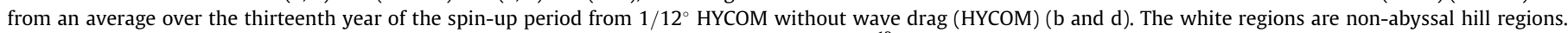
Listed in Table 1 are the globally integrated energy dissipation rates of each estimate $\left[\mathrm{TW}=10^{12} \mathrm{~W}\right]$.

relatively large wave drag dissipation rates found here along the equator with the HYCOM stratification but not with the WOA stratification (Fig. 3). Therefore, we will investigate whether differences between velocity and stratification products can play an important role in the offline wave drag dissipation rate estimates along the equator in Section 4.4.

It is important to note that, as in Nikurashin and Ferrari (2011) and Scott et al. (2011), our offline estimates of wave drag are only active in regions where there are abyssal hills. A globally integrated offline estimate using the G05 scheme (with either abyssal stratification) together with drag fields that are also defined in non-abyssal hill regions yields about three times as much energy dissipation as estimates made only in abyssal hill regions. This is almost entirely owing to large energy dissipation rates found in the Drake Passage, around Iceland, and in the Bering Strait. All of these regions are marked by either relatively strong flow or relatively strong stratification over rough topographic features.

Before moving onto the inline estimates of energy dissipation rates due to wave drag, we compare $\vec{\tau} / \rho$ from (11) with $\left|r_{\text {drag }}\right| \vec{u}_{d}$ from (14) in Fig. 4 using the same abyssal velocity and stratification fields as in Fig. 3a. The largest differences between $\vec{\tau} / \rho$ and $\left|r_{\text {drag }}\right| \vec{u}_{d}$ are not localized to steep topographic ridges, and these differences vary between different choices of five-day averaged abyssal velocity fields. However, Fig. 4 shows many of the typical discrepancies. The differences can be particularly pronounced in the South Indian Ocean (Fig. 4a and b), east of Drake Passage (Fig. 4a and b), around the Gulf Stream (Fig. 4e and f), and between Great Britain and Iceland (Fig. 4e and f). These are regions where the off-diagonal components of $\mathbf{T}$ can be significant enough to rotate the wave drag several degrees from the direction of the abyssal velocities and contract the length of the wave drag vector by several percent. The differences are relatively small elsewhere, such as in the equatorial Pacific Ocean (Fig. 4c and d). While it is important to note that we have only incorporated the contribution of wave drag to the momentum equations in the direction parallel to the flow, the inline calculation adjusts the velocities to the wave drag. The mechanical energy budget, (22), is only affected indirectly by representating $\vec{\tau} / \rho$ with $\left|r_{\text {drag }}\right| \vec{u}_{d}$; thus, this should not act as a missing sink of energy overall. The energy budget will be discussed in more detail in Section 4.5.

\subsection{Inline estimates of the input/output energy budget terms}

In this section, we evaluate two different inline estimates of the input and output terms in the energy budget, (22), one from a simulation without wave drag and one from a simulation with wave drag. For the simulation with wave drag, the G05 scheme is used in conjunction with the inline stratification and velocities from HYCOM. The four different globally integrated energy dissipation rates and the wind power put into the geostrophic and total flows 


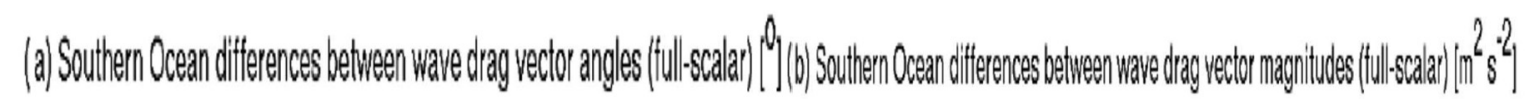

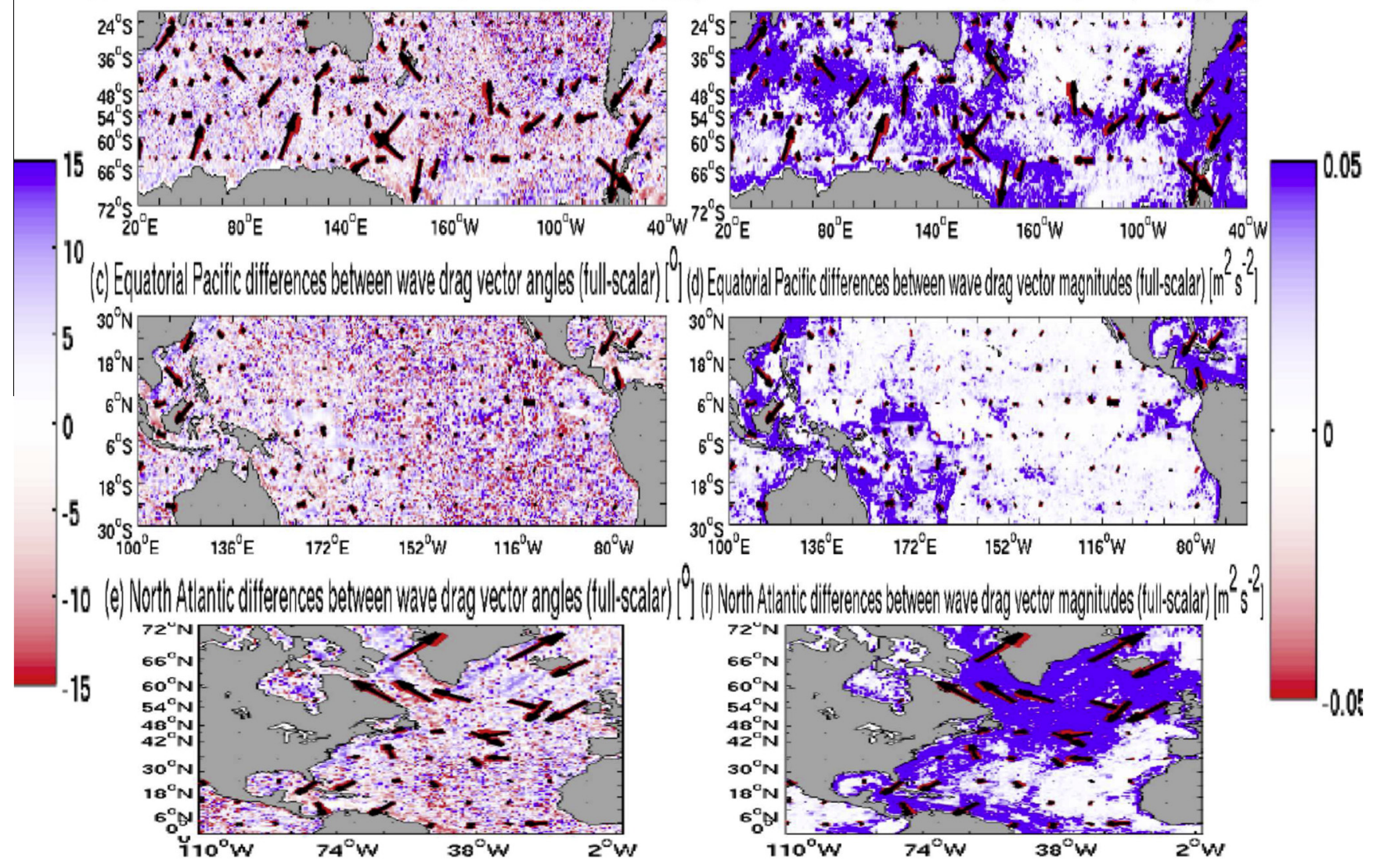

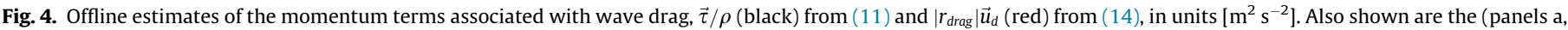

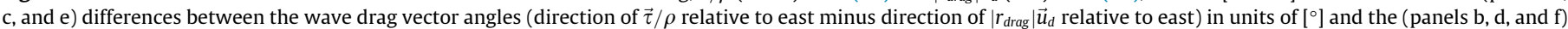

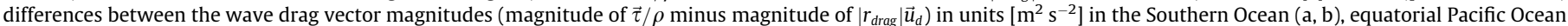

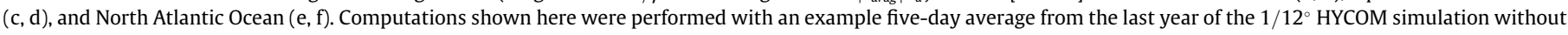
wave drag and the vectors are shown separated by ten degree spacings.

Table 2

The estimated globally integrated energy dissipation rate $\left[\mathrm{TW}=10^{12} \mathrm{~W}\right]$ due to quadratic bottom drag (BD), parameterized internal lee wave drag (WD), vertical viscosity (VV), and horizontal viscosity (HV) averaged over the final year of the spinup (Year) of HYCOM with wave drag and the final year of the spin-up (Year) of HYCOM without wave drag. Also listed are the wind power inputs to the total flow (WindT) and geostrophic flow (WindG), the latter of which excludes the regions within five degrees of the equator.

\begin{tabular}{llllllll}
\hline Wave drag? & Year & WindT & WindG & BD & WD & VV & HV \\
\hline Yes & 20 & 0.868 & 0.626 & 0.140 & 0.402 & 0.275 & 0.257 \\
No & 14 & 0.867 & 0.643 & 0.307 & N/A & 0.286 & 0.294
\end{tabular}

are listed in Table 2. Maps of the wind power put into the geostrophic and total flows are given in Fig. 5a and c, respectively. Differences between the wind power input to the total and geostrophic terms in the simulations with versus without wave drag are shown in Fig. 5b and d, respectively.

The energy inputs (Fig. 5a and c) take on some of their largest values in the western boundary current and ACC regions. The wind power put into the geostrophic flow is about $0.63 \mathrm{TW}, 0.24$ TW less than the wind power input to the total flow (Table 2). Gnanadesikan et al. (2005) find a similar difference between their model-based estimates for the wind power input to the total and geostrophic flows - their Table 1. The total surface velocities and sea surface height distribution are locally altered with the addition of wave drag, as shown in Fig. 5b and d, respectively. However, the global integrals of the wind input into the total and geostrophic flows are not substantially altered. It is possible that the interannual variability in the surface velocities and sea surface height distribution is responsible for the differences we find in Fig. 5b and $d$, but similar differences are found using output from a different year of the simulation with wave drag (not shown).

Because we have not computed the conversion from kinetic to potential energy ${ }^{9}$ in either simulation, and we have not computed the material derivatives of kinetic and potential energy in the simulation without wave drag, we cannot attribute the changing imbalance between inputs and dissipators in (16) upon insertion of wave drag into the model (Table 2) to a particular term. However, as we noted in Section 2, the globally integrated kinetic energy decreases and the globally integrated potential energy increases upon insertion of wave drag. Also, the material derivative of the mechanical energy should asymptote to a relatively small value as the model spins up, which, as we will see in Section 4.5, is the case in the simulation with wave drag. Thus, it is likely that the conversion from kinetic energy to potential energy term in (16) compensates for the increase in net dissipation upon insertion of wave drag, while the wind power input stays relatively constant.

\footnotetext{
${ }^{9}$ We did not compute the conversion from kinetic to potential energy due to difficulties with computing the vertical velocities, which are not required as prognostic variables in hybrid coordinate models, below the surface in HYCOM.
} 

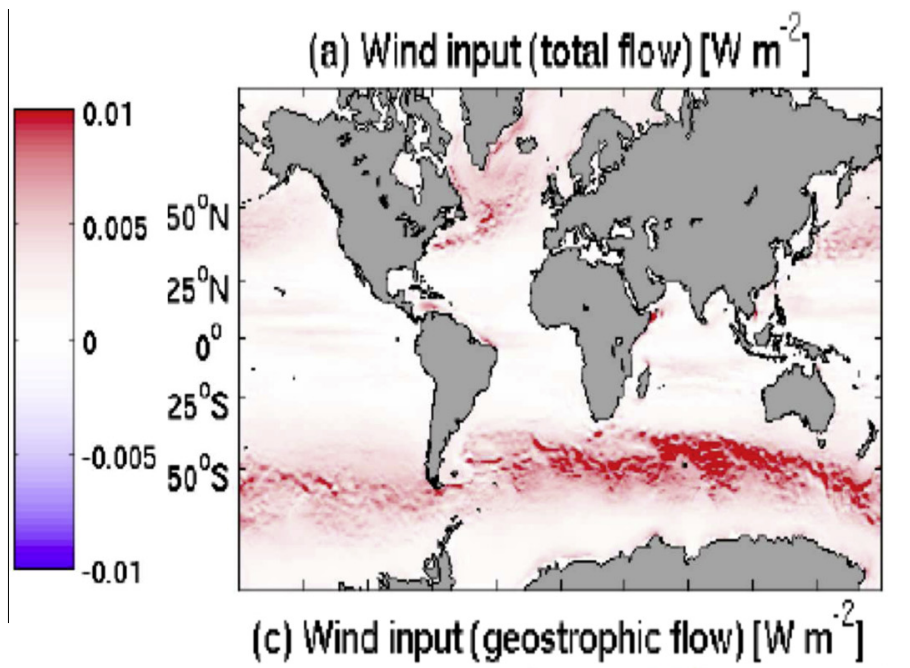

(b) Wind input(total flow) percent difference
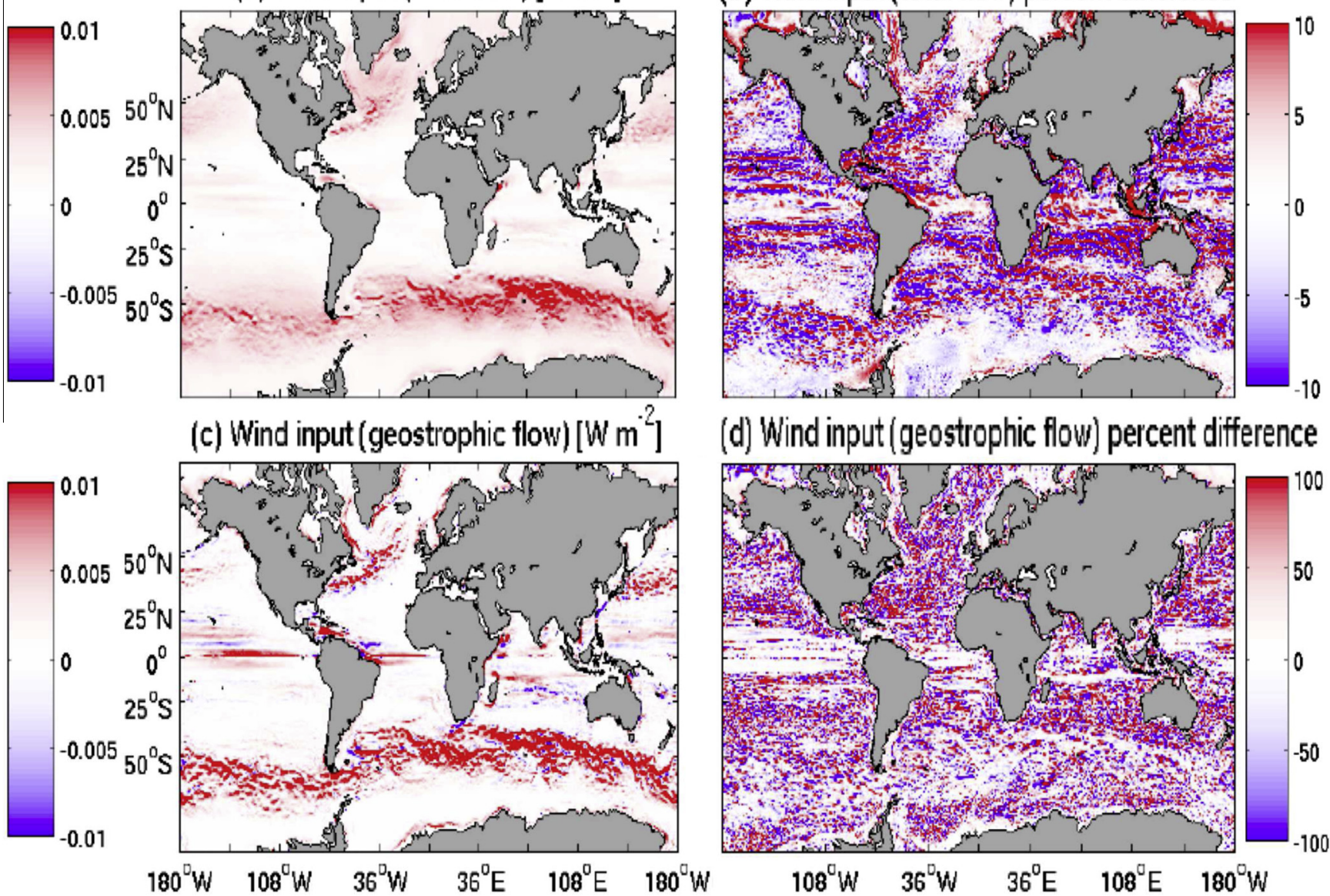

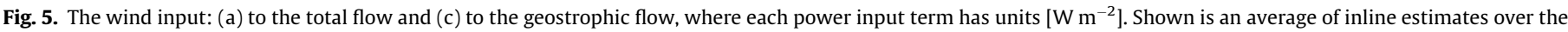

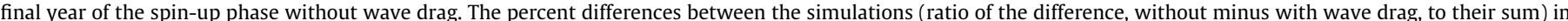

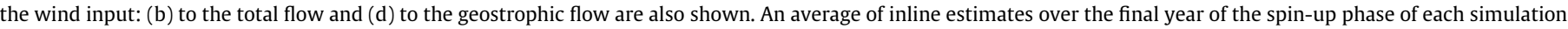
has been used. Listed in Table 2 are the globally integrated power contributions of each term shown in panels a and c.

Some spatial features of the vertical and horizontal viscosity terms are shown in Fig. 6 and discussed below because their large-scale patterns can be physically relevant. Supporting the hypotheses of Thomas and Taylor (2010) and Zhai et al. (2012) that a combination of mixed layer processes (e.g., shear generated in the mixed layer) could leave less energy available to deep ocean mixing than previously thought, we find a relatively large dissipation rate due to vertical viscosity in the mixed layer alone (Fig. 6b). More than three quarters (79.7\%) of the energy dissipated by vertical viscosity is dissipated in the mixed layer. The zonally-averaged viscosity dissipation terms (Fig. 6b and d) in each isopycnal layer have been multiplied by their respective layer thicknesses to make their units consistent with the depth-integrated viscosity dissipation terms (Fig. 6a and c). The vertical viscosity term is largest in equatorial regions (Fig. 6a), in the intensified jets (Fig. 6a), and in the mixed layer (Fig. 6b). The horizontal viscosity term is largest along the fringes of the intensified jets (Fig. 6c), in the deep convection and overflow regions (Fig. 6c), and along bathymetric boundaries (Fig. 6d). The vertical viscosity term is always largest in the hemisphere that is experiencing wintertime conditions (which can be inferred from Fig. 7) and is the only dissipative term that seasonally varies by an order of $0.1 \mathrm{TW}$, the same order of magnitude of the seasonal variability in the wind input (Fig. 7). Evidence of deep convection (and the formation of overflow waters in the northern hemisphere) can clearly be seen in the plumes of large zonally averaged vertical viscosity dissipation rates that go to about $2000 \mathrm{~m}$ depth around $50^{\circ}-55^{\circ} \mathrm{N}$ and about $3000 \mathrm{~m}$ depth around $55^{\circ}-60^{\circ} \mathrm{S}$ (Fig. 6b). Large zonally averaged horizontal viscosity dissipation rates outline the regions of deep convection and formation of overflow waters (Fig. 6d).

We find that point estimates of both of the viscosity terms can be unphysical (i.e., that there are single grid points that suggest dissipative processes are adding energy to the system). Shown in Fig. 6 are the negative of each of the viscosity terms, so a positive viscosity dissipation, for example, is shown as a negative value in Fig. 6. At a single grid point, there is no physical meaning to a horizontal viscosity, let alone energy dissipation rate due to horizontal viscosity (Fox-Kemper and Menemenlis, 2008). As with the horizontal viscosity term, the vertical viscosity term can be either positive or negative locally, but is negative-definite when globally integrated. The vertical viscosity term is positive following the jet of the ACC near the surface (Fig. 6a), the regions and depths in the northern hemisphere to which water subducts, and in the locations of Antarctic Bottom Water (Fig. 6b). The horizontal viscosity term has some positive values along the coasts and in the middle of the open ocean (Fig. 6c). Also, it can be argued that, at coarser horizontal grid resolutions, the larger mid-depth horizontal viscosity required leaves less energy to be dissipated in 

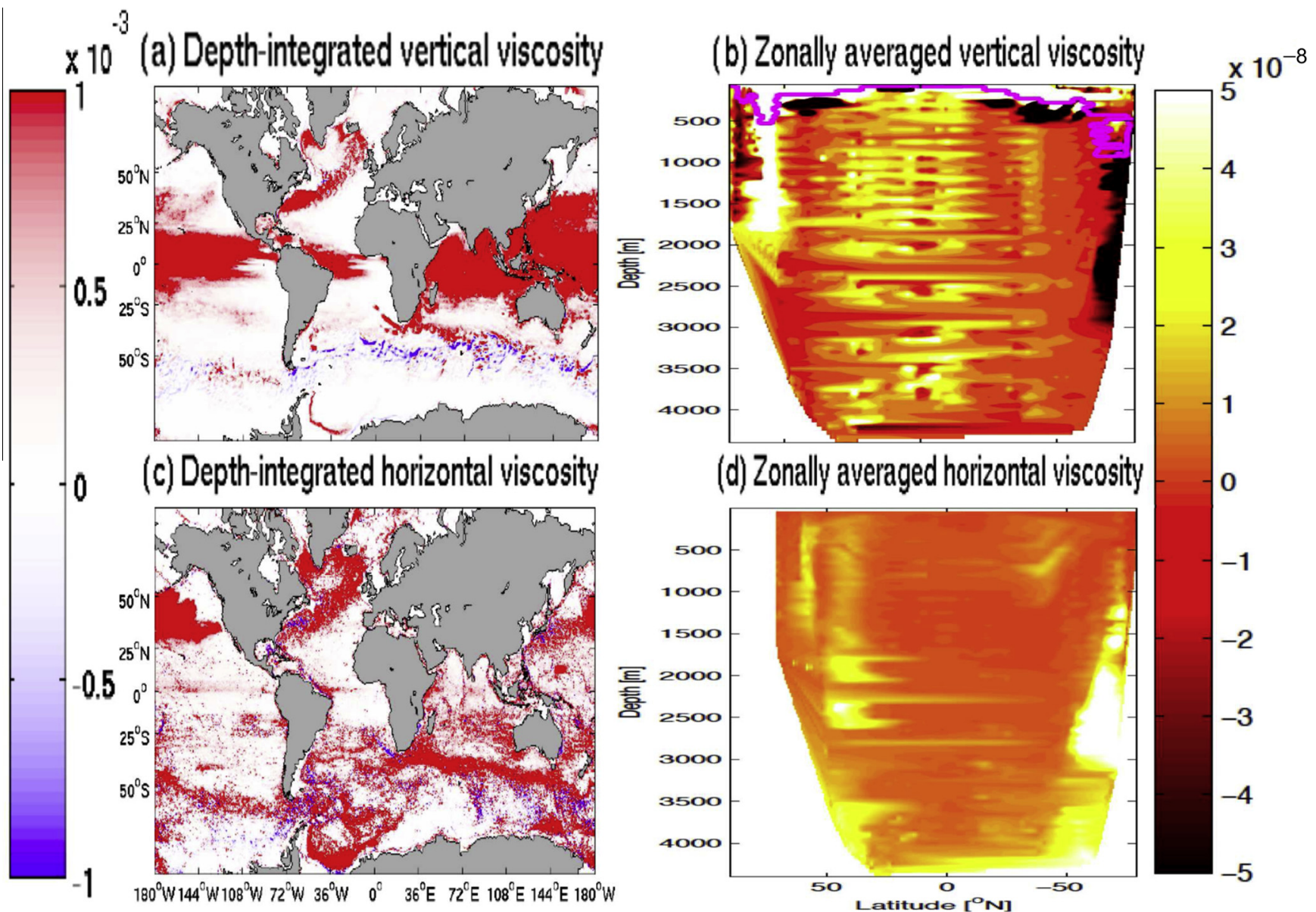

(d) Zonally averaged horizontal viscosity
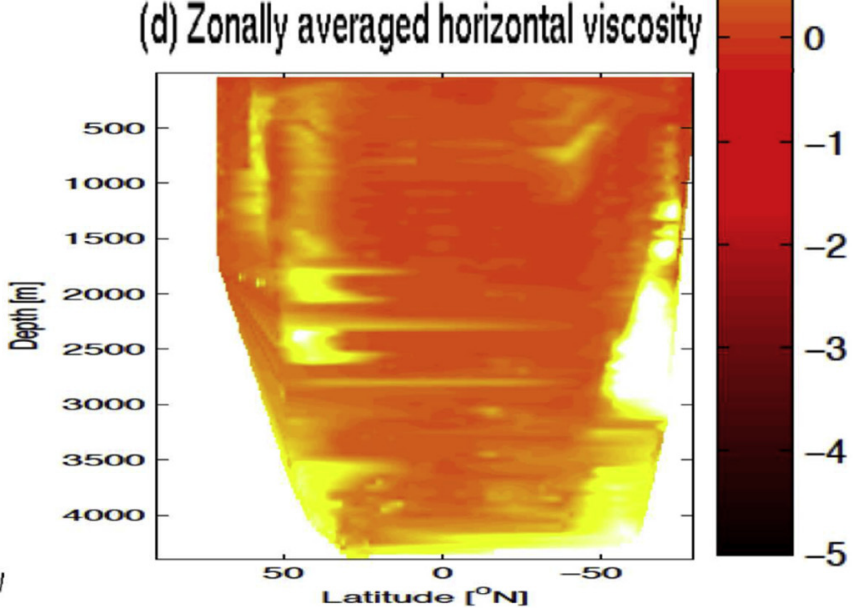

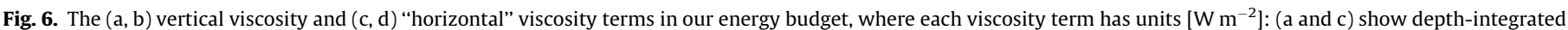

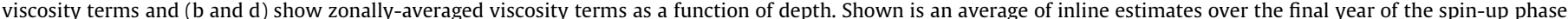

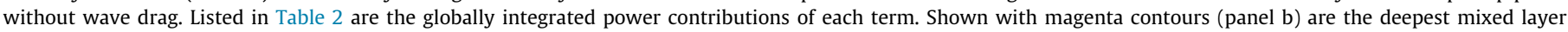
depths, as determined by KPP, from the final year of the $1 / 12^{\circ}$ HYCOM simulation without wave drag.

the bottom, thus implying weaker abyssal velocities. The same argument can be applied to coarser vertical grid resolutions, where the larger vertical viscosity required near the surface leaves less energy to be dissipated in the abyss, thus implying weaker abyssal velocities. This suggests that in simulations with higher grid resolutions, our estimates of bottom drag and wave drag dissipation rates should increase, but only up to a point. This will be investigated in a future study.

Differences between the vertical and horizontal viscosity terms with and without wave drag are shown in Fig. 8. In the simulation with wave drag, the vertical viscosity term is smaller in regions where deep convection occurs but larger in the mixed layer when compared to the simulation without wave drag (Fig. 8a and b). The horizontal viscosity term is larger in regions where deep convection occurs but smaller near the seafloor in the simulation with wave drag than in the simulation without wave drag (Fig. 8c and d). However, in both simulations, the global integral of the horizontal viscosity term is comparable in magnitude to that of the vertical viscosity term (Table 2). While Fig. 8 suggests that the partition of energy is altered locally when wave drag is inserted, Table 2 suggests that, with the exception of the bottom drag term, the partition of energy dissipated by viscosity is only marginally impacted globally when wave drag is inserted.

The differences between our vertical viscosity dissipation rates with and without wave drag (Fig. 8a and b) suggest that adding

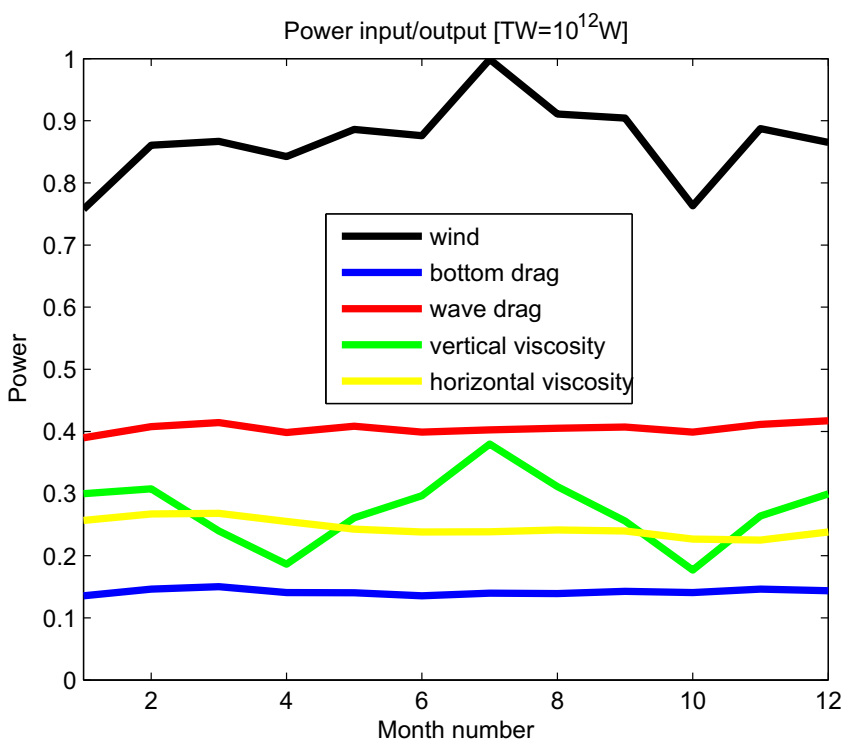

Fig. 7. The seasonal cycle of the estimated energy dissipation rate [TW $\left.=10^{12} \mathrm{~W}\right]$ due to quadratic bottom drag, parameterized internal lee wave drag, vertical viscosity, and horizontal viscosity, from the last year of a climatologically forced spin-up of HYCOM with wave drag, as a function of climatological month. Also shown is the wind power input to the total flow. Here, ' 1 ' refers to January,..., and ' 12 ' refers to December. 
(a) Depthintegrated vertical viscosity difference (without-with wave drag)

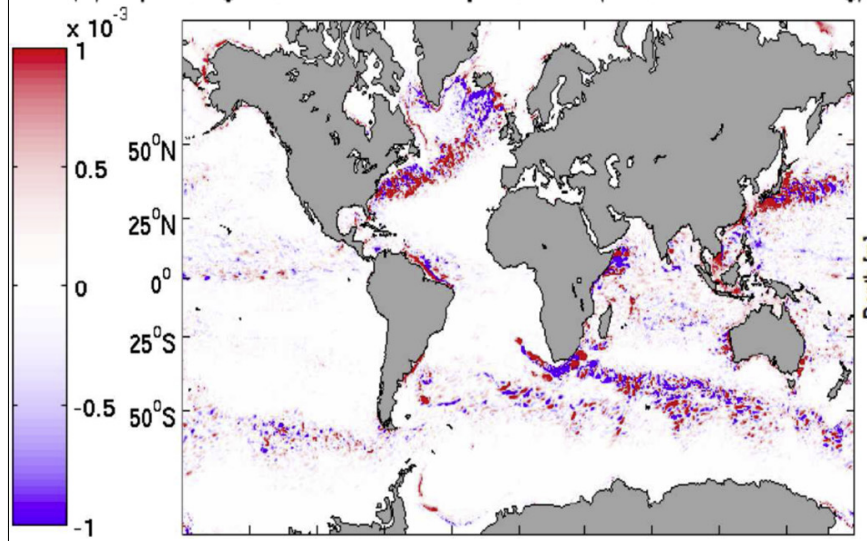

(b) Zonally averaged vertical liscosity difference (without-with wave drag)

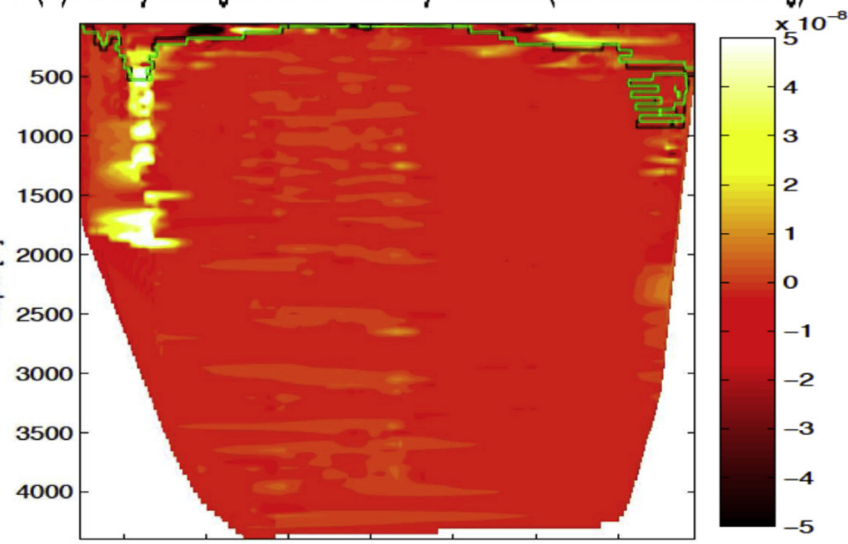

(c) Depth-integrated horizontal viscosity difference (without-with wave drag)
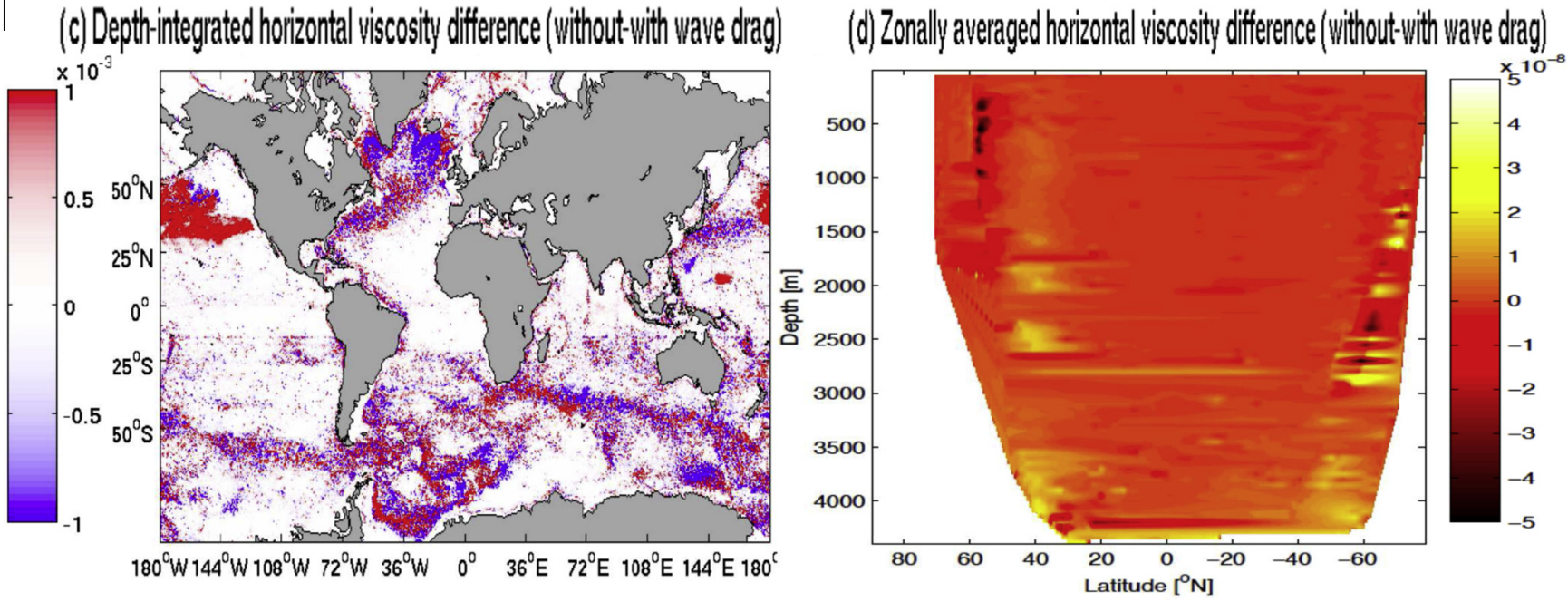

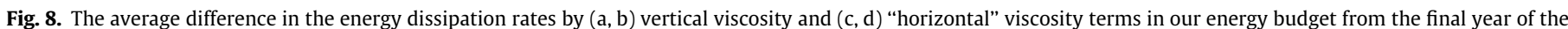

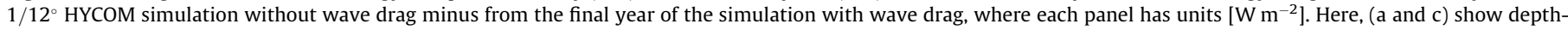

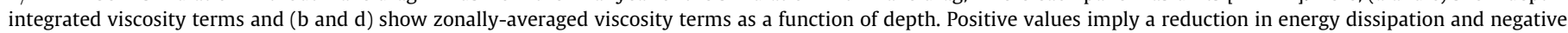

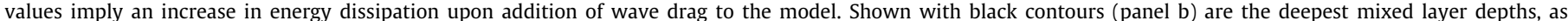

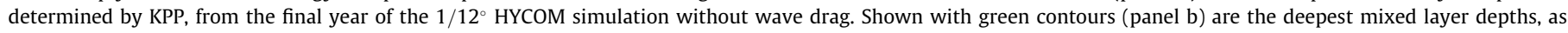
determined by KPP, from the final year of the $1 / 12^{\circ}$ HYCOM simulation with wave drag.

wave drag to the momentum equations enhances the vertical diffusivity in a number of locations that previous studies have examined. ${ }^{10}$ For example, Polzin et al. (1997) and St. Laurent et al. (2012) found enhanced levels of diapycnal diffusivity above rough topography in the Brazil Basin and in the Drake Passage, respectively. Also, Ledwell et al. (2000) and Kunze et al. (2006) found enhanced levels of energy dissipation above rough topography around the northern portion of the Mid-Atlantic Ridge and just south of Iceland.

Our bottom drag dissipation rates are more comparable to those from previous studies when we insert wave drag. Maps of the bottom drag and wave drag dissipation rates per unit area are given in Fig. 9a and c, respectively. Maps of the coefficients associated with bottom drag $\left(C_{d}\left|\overrightarrow{u_{b}}\right|\right)$ and wave drag $\left(r_{d r a g}\right)$ are given in Fig. 9b and d, respectively. While we find bottom drag dissipation rates in the simulation without wave drag ( $0.31 \mathrm{TW}$; see Table 2$)$ to be larger than a previous estimate of 0.2 TW based on an observationally-informed least-squares regression fit (Sen et al., 2008), our energy dissipation rates due to bottom drag are smaller than this previous estimate when wave drag is inserted (0.14 TW; see Table 2). Our

\footnotetext{
10 Each component of $\left(\partial v_{z} / \partial z\right)(\partial \vec{u} / \partial z)$ tends to be at least an order of magnitude less than each component of $v_{z}\left(\partial^{2} \vec{u} / \partial z^{2}\right)$ in (17) which suggests that we may interpret the relative magnitude of the vertical viscosity term in our energy budget as a relative magnitude of the vertical diffusivity.
}

energy dissipation rates due to bottom drag in the simulation without wave drag are larger than the Arbic et al. (2009) estimates based on two non-data-assimilative high-resolution models, but our bottom drag estimates are comparable to the estimates of $\mathrm{Ar}$ bic et al. (2009) when wave drag is inserted. Our energy dissipation rates due to bottom drag may be larger than those of Aiki et al. (2011) because Aiki et al. (2011) defined bottom friction relative to the average vertical mixing (as opposed to the quadratic drag used in our model). The bottom drag and wave drag terms in our energy budget are large in the intensified jet regions, in the overflow regions around Iceland, in the Labrador Sea, on the coastal margins of the Arctic Ocean north of Asia, around the Nordic Seas, and in the Bering Strait (Fig. 9a and c).

The percent differences between the offline and inline estimates of dissipation rates associated with wave drag are shown in Fig. 10. About half of the dissipation associated with wave drag occurs in regions where there are no abyssal hills, which increases the globally integrated dissipation rate, but the local dissipation rates due to wave drag are generally smaller when calculated inline instead of offline (Fig. 10). In regions where there are abyssal hills, the inline wave drag energy dissipation rates (Fig. 9c) are spatially similar to those from offline estimates (Fig. 3b) with one noteworthy exception. The dissipation rates are substantially reduced along 
(a) Bottom drag $\left[\log _{10}\left(\mathrm{~W} \mathrm{~m}^{-2}\right)\right]$

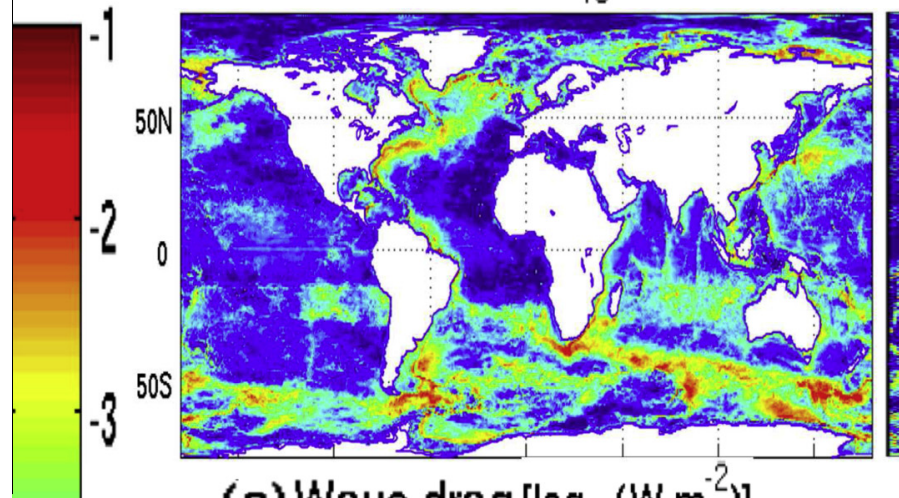

(c) Wave drag $\left[\log _{10}\left(\mathrm{~W} \mathrm{~m}^{-2}\right)\right]$

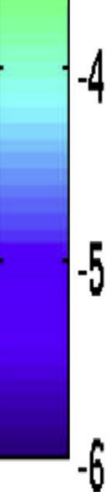

$\left\{\begin{array}{l}-4 \\ -5 \\ -6\end{array}\right.$

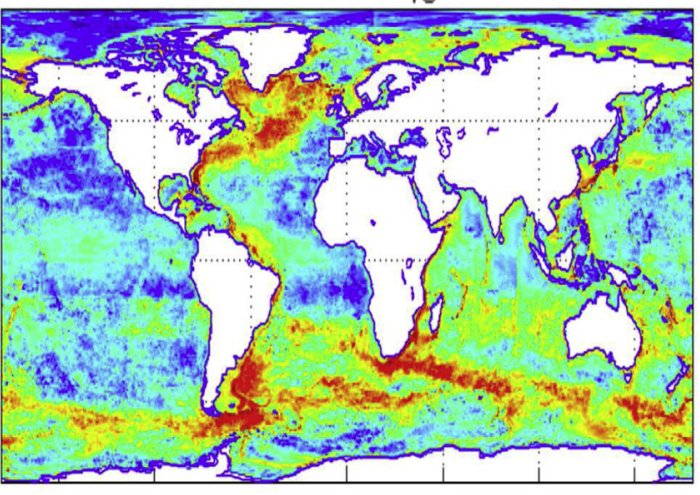

150W 100W 50W O 50E 100E 150E (b) $C_{d}\left|u_{b}\right|\left[\log _{10}\left(m^{-1}\right)\right]$

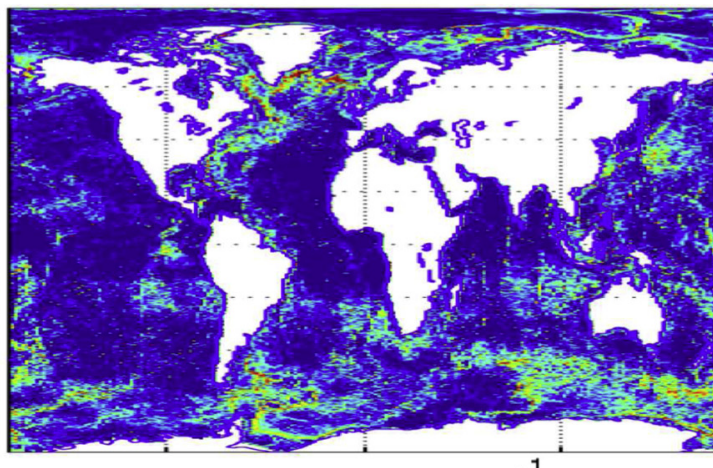

(d)

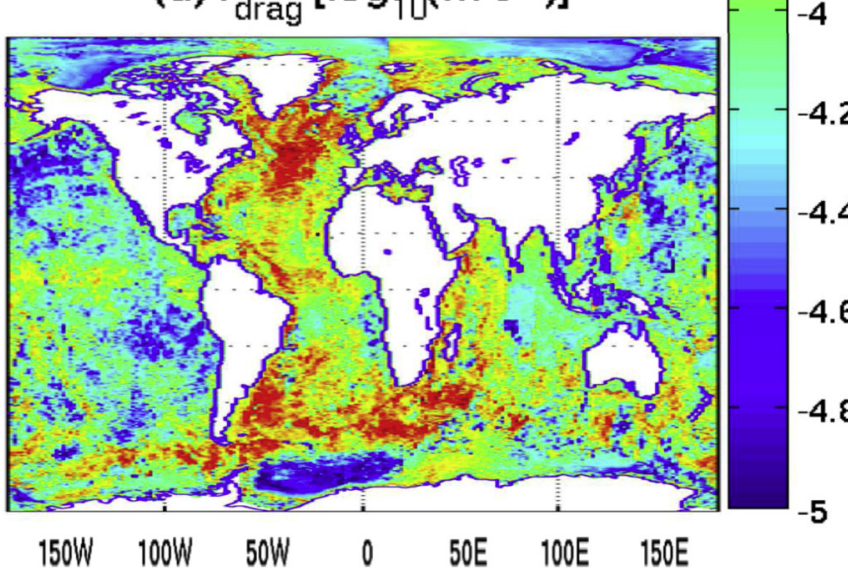

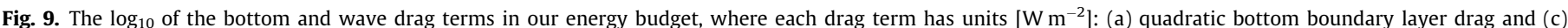

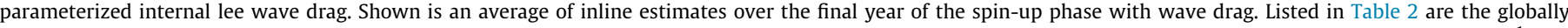

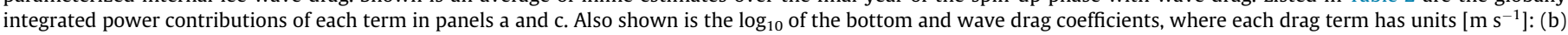
$C_{d}\left|\vec{u}_{b}\right|$ and (d) $r_{\text {drag }}$.

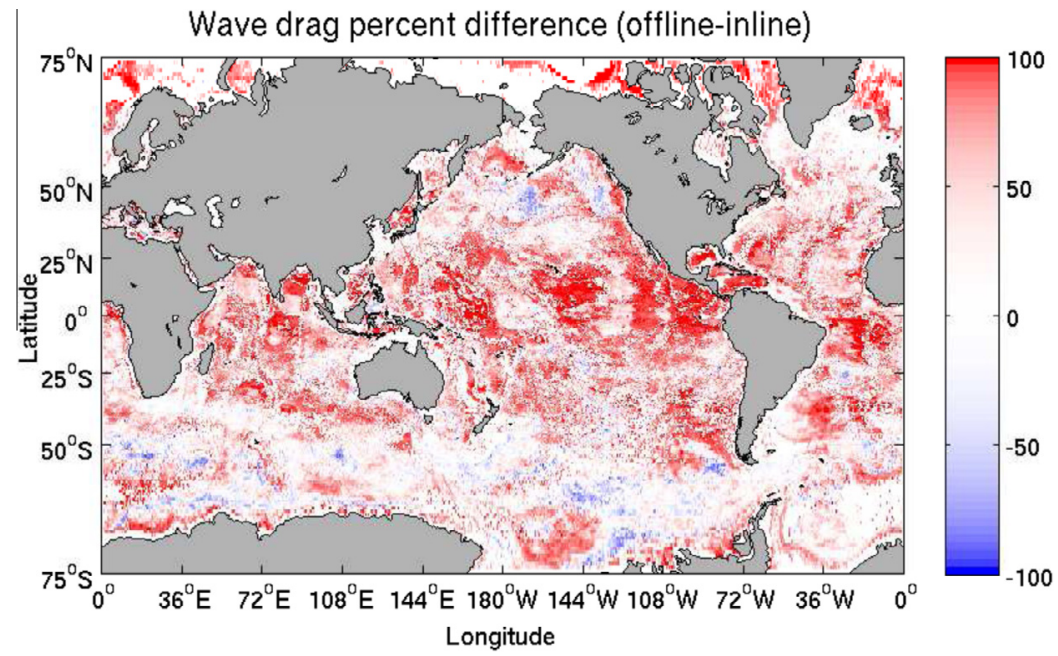

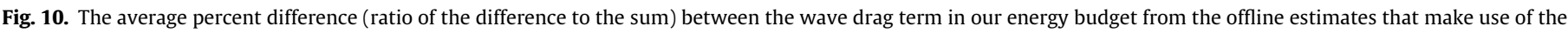

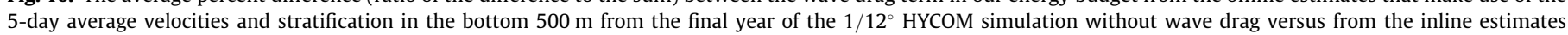
computed during every 2 -min baroclinic time step during the final year of the $1 / 12^{\circ}$ HYCOM simulation with wave drag.

the equator when wave drag is inserted into the model. A comparison of the dissipation rates from Nikurashin and Ferrari (2011) versus those in Scott et al. (2011) and in our offline estimates suggests that allowing for an anisotropic spectral form of the abyssal hill power spectrum yields smaller dissipation rates along the equator than use of an isotropic spectral form of the abyssal hill power spectrum. Fig. 10 suggests that putting wave drag into a model reduces the dissipation rates by up to an order of magnitude 


\section{(a) Bottom $N$ without wave drag $\left[\log _{10}\left(s^{-1}\right)\right]$}

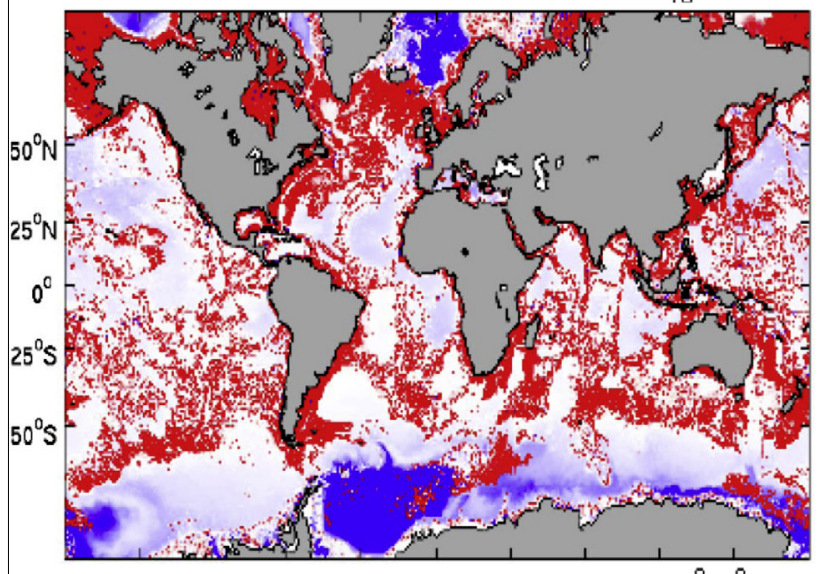

(c) Bottom KE without wave drag $\left[\log _{10}\left(\mathrm{~m}^{2} \mathrm{~s}^{-2}\right)\right]$

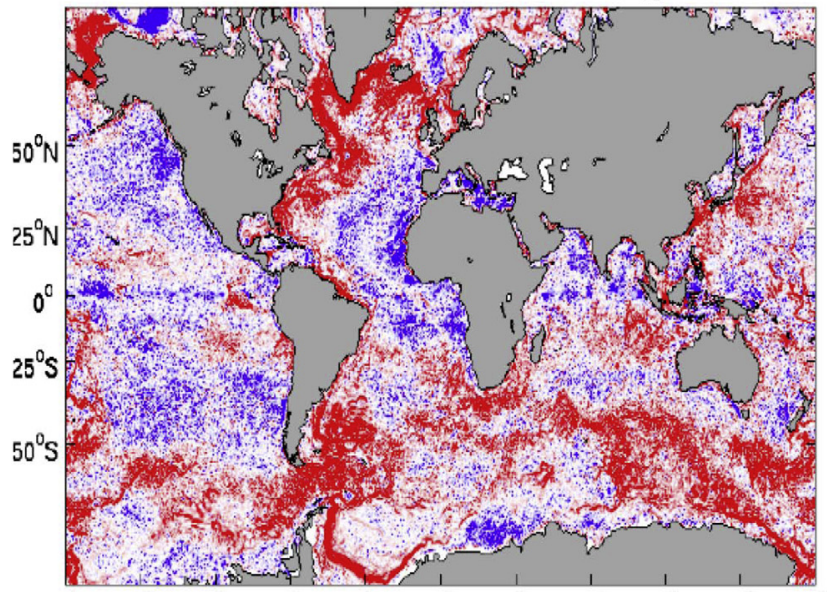

(b) Bottom $N$ with wave $\operatorname{drag}\left[\log _{10}\left(s^{-1}\right)\right]$

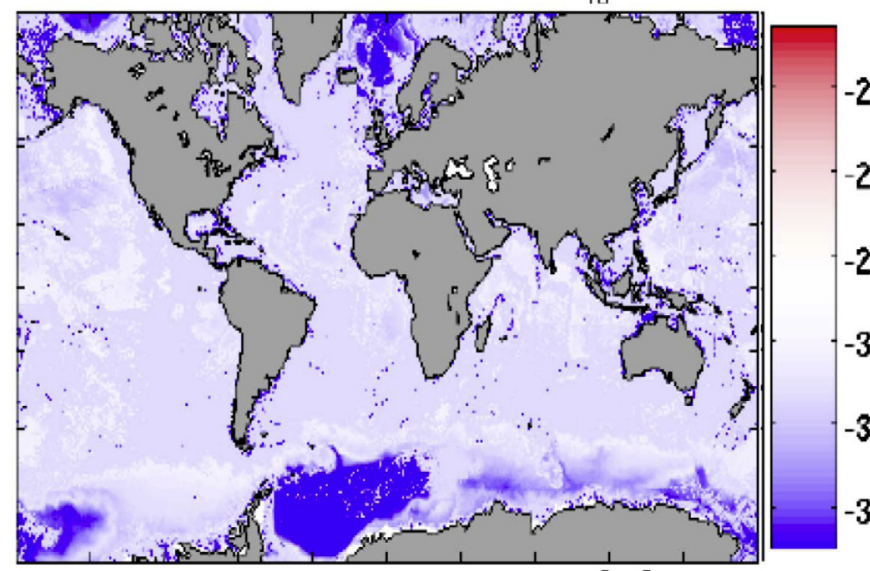

(d) Bottom KE with wave drag $\left[\log _{10}\left(m^{2} s^{-2}\right)\right]$

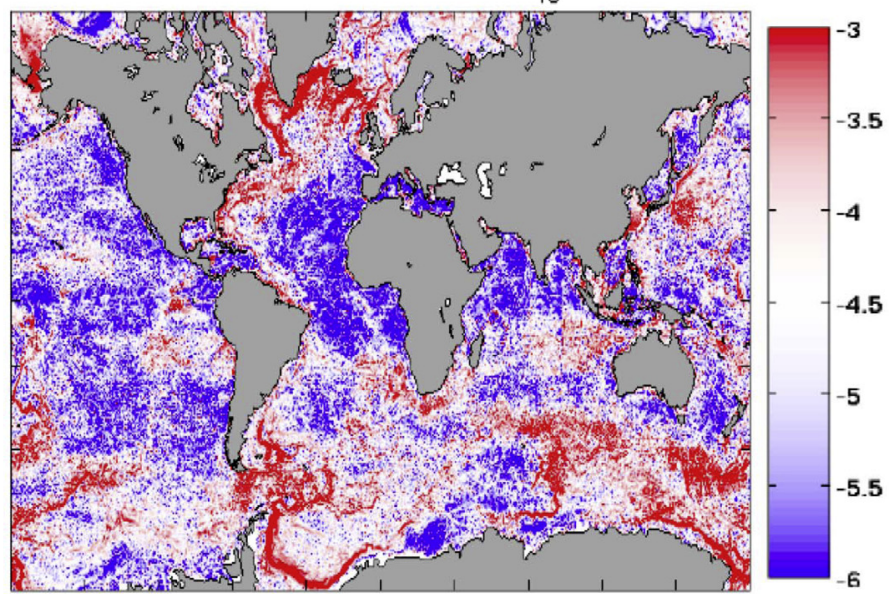

$180^{\circ} \mathrm{W} 144^{\circ} \mathrm{W} 108^{\circ} \mathrm{W} 72^{\circ} \mathrm{W} \quad 36^{\circ} \mathrm{W} \quad 0^{\circ} \quad 36^{\circ} \mathrm{E} \quad 72^{\circ} \mathrm{E} 108^{\circ} \mathrm{E} 144^{\circ} \mathrm{E} 180^{\circ} 180^{\circ} \mathrm{W} 144^{\circ} \mathrm{W} 108^{\circ} \mathrm{W} 72^{\circ} \mathrm{W} 36^{\circ} \mathrm{W} \quad 0^{\circ} \quad 36^{\circ} \mathrm{E} \quad 72^{\circ} \mathrm{E} 108^{\circ} \mathrm{E} 144^{\circ} \mathrm{E} 180^{\circ} \mathrm{W}$

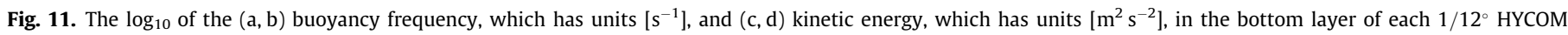
simulation, averaged over the last year of the spin-up: (a and c) without wave drag and (b and d) with wave drag.

in equatorial regions. As far as the authors and E. Kunze (personal communication) are aware, there is no evidence for enhanced dissipation near the seafloor at the equator relative to surrounding latitudes. Thus, our inline estimates at the equator may be an improvement upon the offline estimates. The regions where the dissipation rates are increased when wave drag is inserted into the model relative to the offline estimates are in the ACC, some extreme latitudes in the Southern Hemisphere, and the North Pacific Ocean. ${ }^{11}$ The net effect is that the inline wave drag dissipation rate (about $0.4 \mathrm{TW}$ ) has a relatively small global integral compared to the offline estimates (about 1.2 TW from the offline calculation over all regions using the HYCOM stratification).

Previous studies have noted the possibility that bottom drag may substitute for wave drag in models without a wave drag parameterization (Arbic and Flierl, 2004; Wright et al., 2013). The visually apparent correlation between bottom drag and wave drag in Fig. 9a and c suggests that bottom drag may be a plausible substitute for wave drag. A least squares linear regression fit (not shown), regressing energy dissipation rates due to bottom drag on those due to wave drag, suggests that multiplying the energy dissipation rates

\footnotetext{
11 One reason for this is that, because the velocities are not linearly related to the wave drag, using a five-day average of two-minute varying velocities to calculate the dissipation rates can lead to a net bias.
}

due to bottom drag by a factor of about five would be a substitute for putting an active wave drag scheme in an ocean model. However, the globally integrated energy dissipation rates due to bottom drag are about a factor of about 2.5 times less than the globally integrated energy dissipation rates due to wave drag. This is because the least squares linear regression fit is influenced primarily by the locations where there are large energy dissipation rates due to both bottom drag and wave drag. Where energy dissipation rates due to wave drag are below about $10^{-4} \mathrm{~W} \mathrm{~m}^{-2}$, the approximately linear relationship no longer holds (not shown). At locations with weak wave drag dissipation, bottom drag can be strong (e.g., northeast of Russia in Fig. 9a and b). There are additional reasons for the fact that bottom drag cannot serve as a perfect substitute for wave drag. Bottom drag dissipation is proportional to $\left|\vec{u}_{b}\right|^{3}$, while wave drag dissipation is proportional to $\left|\vec{u}_{d}\right|^{2}$. Furthermore, $C_{d}\left|\vec{u}_{b}\right|$ is completely determined by the velocities in the bottom $H_{B D}$ meters, while $r_{d r a g}$ is determined from properties related to the underlying topography, the stratification, and the velocities in the bottom $H_{W D}$ meters. Note also that $H_{B D} \neq H_{W D}$.

In contrast to the $100 \%$ local dissipation due to bottom drag, Waterman et al. (2013) found a mismatch between energy generation and near-bottom dissipation and suggested that this is because about $80 \%$ of the energy is dissipated non-locally. They used observed abyssal stratification and abyssal velocities north of the Kerguelen Island region to perform the same offline analysis 
(a) Zonally averaged $\mathrm{N}$ without wave drag $\left[\log _{10}\left(\mathrm{~s}^{-1}\right)\right]$ (b) Zonally averaged $\mathrm{N}$ difference (without-with wave drag)

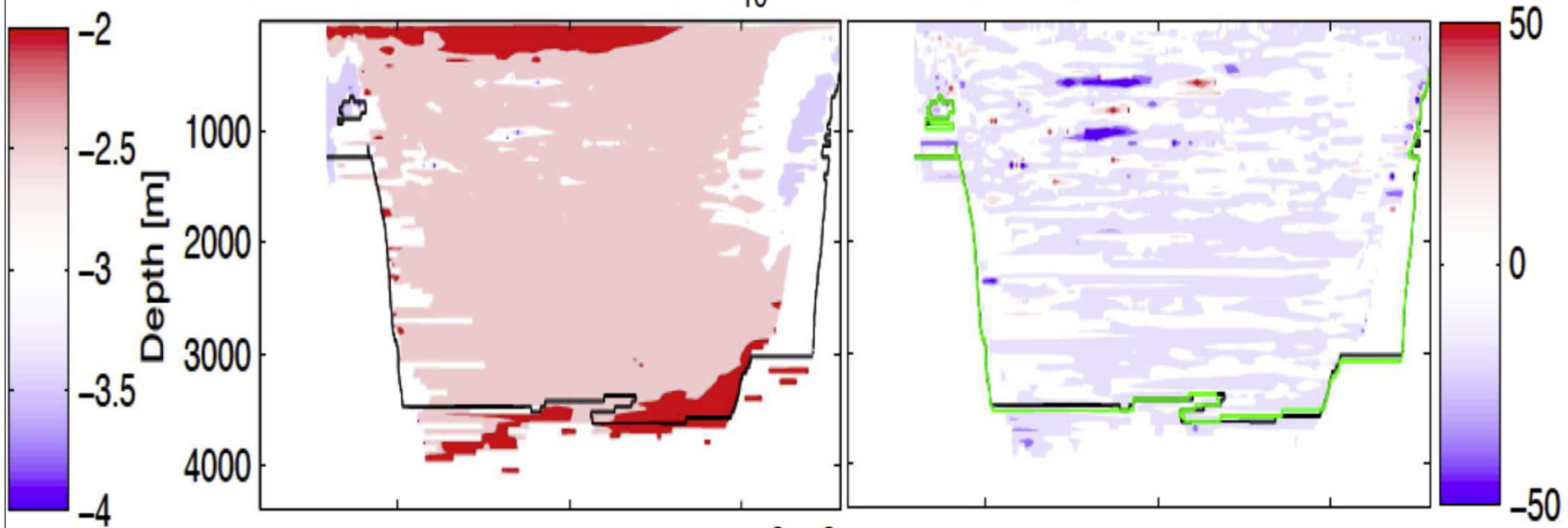

(c) Zonally averaged KE without wave drag $\left[\log _{10}\left(\mathrm{~m}^{2} \mathrm{~s}^{-2}\right)\right](\mathrm{d})$ Zonally averaged KE difference (without-with wave drag)

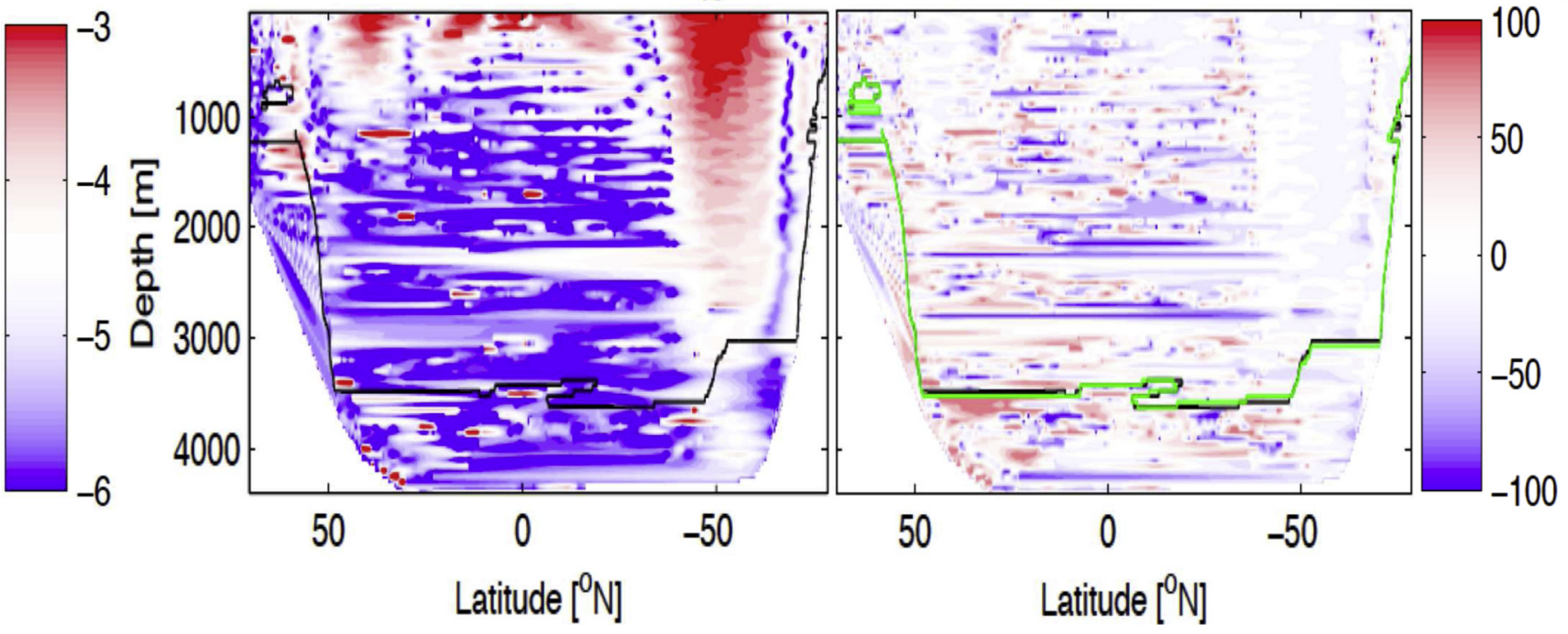

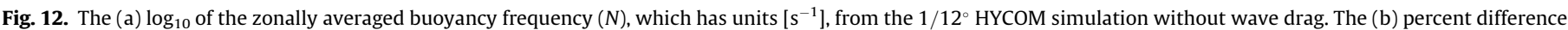

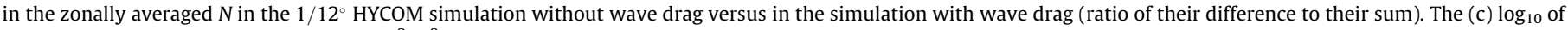

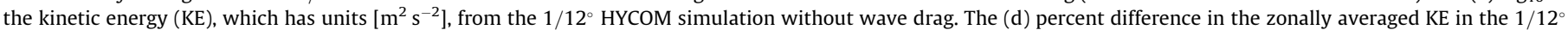

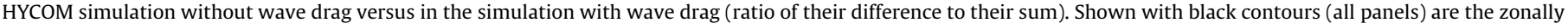

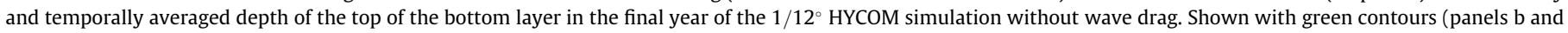
d) are the zonally and temporally averaged depth of the top of the bottom layer in the final year of the $1 / 12^{\circ} \mathrm{HYCOM}$ simulation with wave drag.

as Nikurashin and Ferrari (2011). However, the isotropic form of the abyssal hill power spectra used by Nikurashin and Ferrari (2011) and Waterman et al. (2013) contrast with the anisotropic power spectra employed by Scott et al. (2011) and in the present manuscript. Also, we find that the inline energy dissipation due to wave drag is reduced by an order of magnitude when compared to the offline estimates in the region studied by Waterman et al. (2013). This raises the question of whether discrepancies between offline energy dissipation estimates and observed near-bottom dissipation are entirely due to non-local energy dissipation or to more subtle issues with how the energy dissipation rates are estimated. Non-local dissipation of energy due to wave drag will be the subject of investigation in a future study.

\subsection{Impact of wave drag on the abyssal currents and stratification}

The inline wave drag estimates are smaller than the fully global offline wave drag estimates (i.e., the offline estimates that include both abyssal hill and non-abyssal hill regions), in part, because of the active feedback that generally reduces the bottom stratification in the model (Fig. 11a and b), particularly in the Arctic and in regions where wave drag is strong. Inline wave drag also reduces the bottom current speeds in the model, as demonstrated in Fig. 11c and d. The area-averaged bottom buoyancy frequency and kinetic energy in the simulation with wave drag are reduced by $59.5 \%$ and $58.5 \%$, respectively, from those in the simulation without wave drag. The bottom stratification (Fig. 12a) and kinetic energy (Fig. 12c) are reduced, on average, when wave drag is inserted, but they increase in some locations. The locations with larger bottom kinetic energy in the simulation with wave drag are in the Arctic. The locations with larger bottom stratification in the simulation with wave drag (Fig. 11a and b) are on the slopes of topographic features that stand a few kilometers higher than their base (Fig. 2c), particularly in the Weddell Sea and near the Hawaiian Ridge.

The zonally averaged buoyancy frequency and kinetic energy are generally reduced closer to the seafloor and enhanced above the seafloor. The differences in the zonally averaged buoyancy frequency and kinetic energy when wave drag is inserted are shown in Fig. 12b and d, respectively. The zonally averaged buoyancy frequency in the simulation with wave drag is enhanced by an aver- 


\section{(a) Offline wave drag percent difference (WOA N, without-with wave drag)}
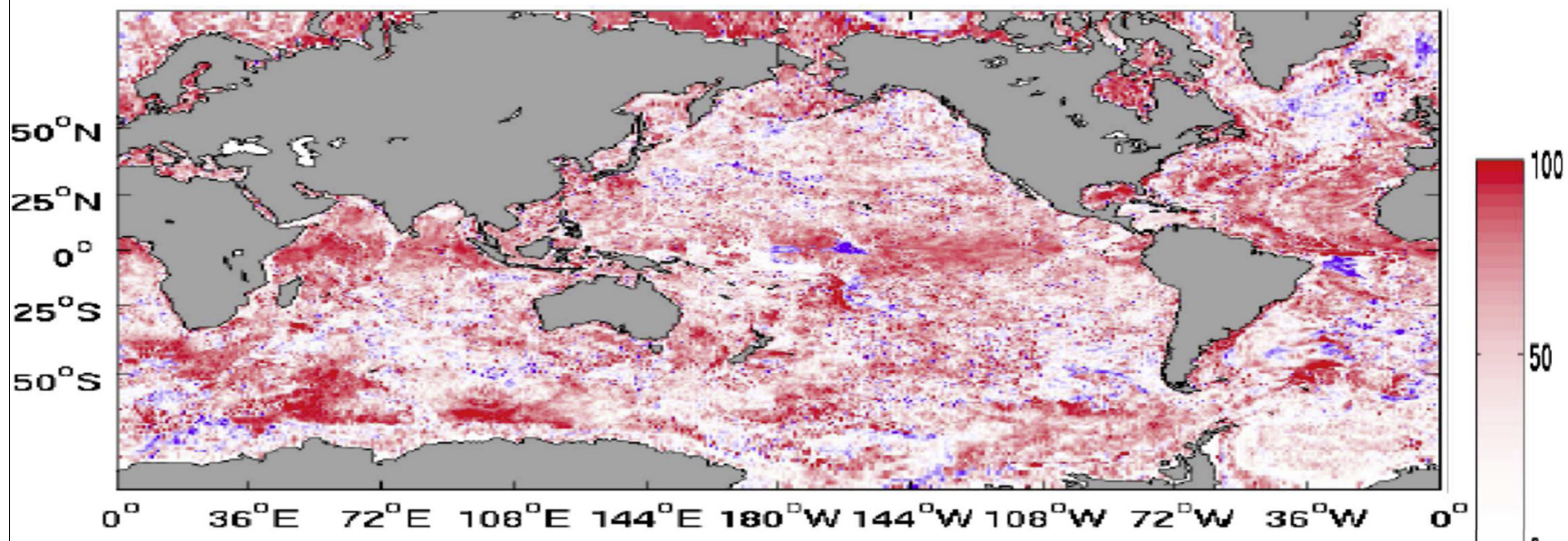

\section{(b) Offline wave drag percent difference (HYCOM N, without-with wave drag)}

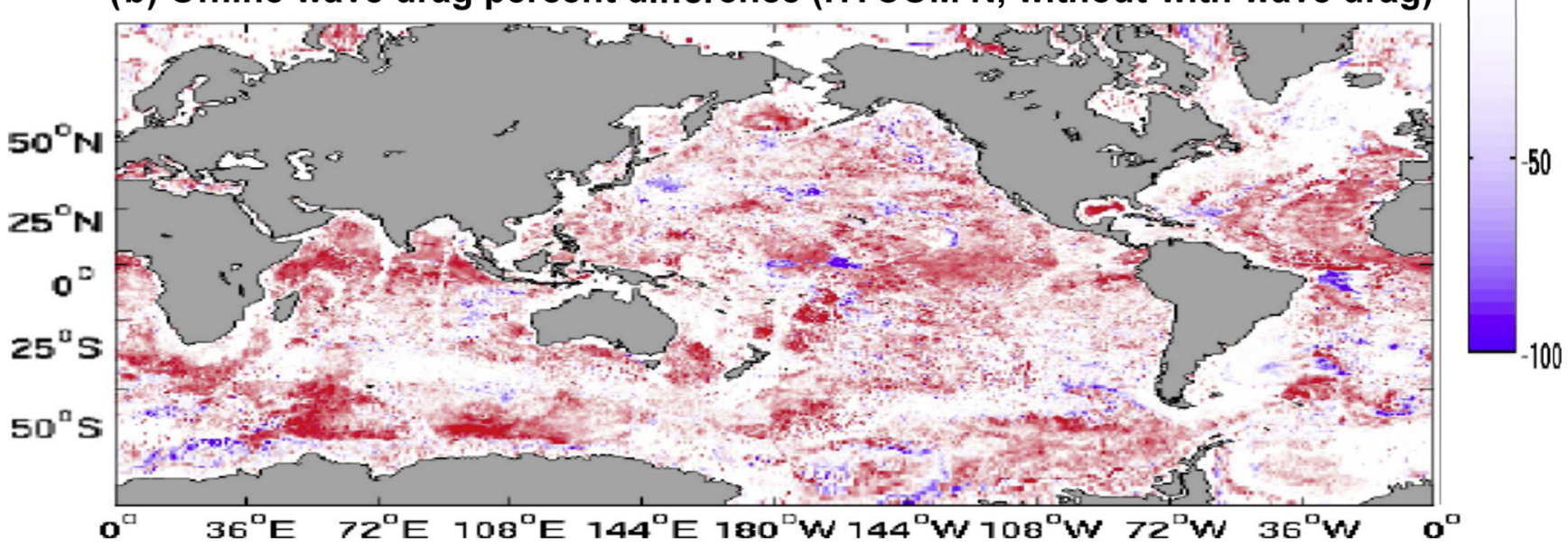

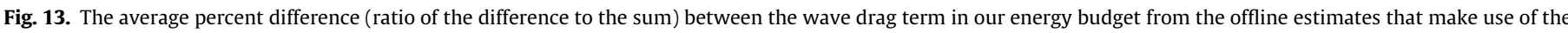

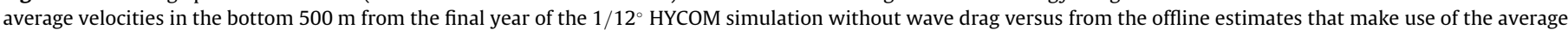

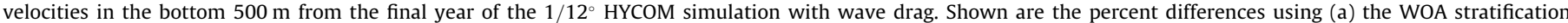
(stratification held constant) and (b) the HYCOM stratification from each respective run (stratification varied).

age of $1.29 \%$ from that in the simulation without wave drag. The zonally averaged kinetic energy in the simulation with wave drag is reduced by an average of $28.0 \%$ from that in the simulation without wave drag. The relatively coarse vertical resolution of the WOA product makes it difficult to compare the bottom stratification in the simulations with and without wave drag (Fig. 11a and b) with that from the observations (Fig. 2a). Comparison of the model with and without wave drag to various observational datasets will be the subject of a future study.

While the primary reason for the overall reduction in bottom kinetic energy is that wave drag acts to remove momentum in the bottom $500 \mathrm{~m}$, the reason for the overall reduction in bottom stratification is less clear. A plausible hypothesis for the reduction in bottom stratification could be that inserting wave drag changes the kinetic energy, which in turn changes the Richardson number in the bottom $500 \mathrm{~m}$. That ultimately enhances the vertical diffusivity via KPP and decreases the abyssal stratification. Evidence for this is shown in Fig. 8b, which generally shows a small increase in energy dissipation associated with vertical viscosity when wave drag is inserted. Additionally, a decrease in the along-isopycnal diffusion could result in less production of heavier waters via cabbeling or thermobaricity, thereby reducing the density contrast between layers at the bottom. Evidence for this is shown in Fig. $8 \mathrm{~d}$ near the seafloor where there is a substantial decrease in energy dissipation associated with horizontal viscosity when wave drag is inserted. Using models that resolve sub-mesoscale motions,

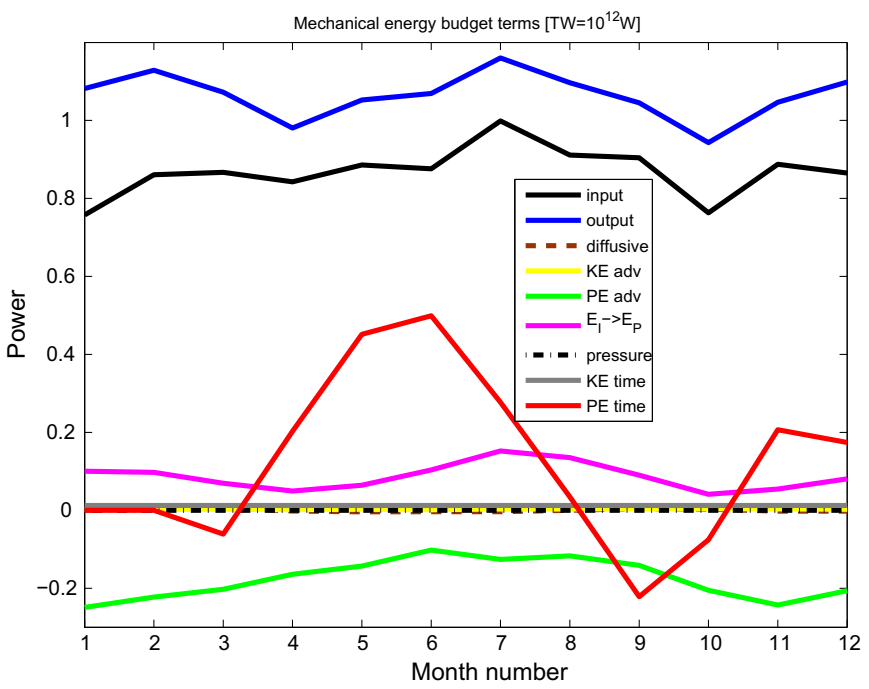

Fig. 14. The seasonal cycle of the estimated power $\left[\mathrm{TW}=10^{12} \mathrm{~W}\right]$ associated with the partial time derivative of kinetic energy $\left(P_{E_{K} t i m e}\right.$; "KE time" in legend), the advective flux of kinetic energy ( $P_{E_{K} a d v}$; “KE adv"), the partial time derivative of potential energy $\left(P_{E_{p} t i m e}\right.$; "PE time"), the advective flux of potential energy $\left(P_{E_{p} a d v}\right.$; "PE adv"), pressure work ( $P_{\text {pressure }}$; "pressure"), buoyancy diffusion $\left(P_{\text {diffusive }}\right.$; "diffusive"), conversion from internal energy to potential energy $\left(C_{E_{1}-E_{P}} ;\right.$; $E_{I}->E_{P}$ "), input ( $P_{\text {input }}$; “input"), and output ( $P_{\text {output }}$; “output") from the last year of a climatologically forced spin-up of HYCOM with wave drag as a function of climatological month. Here, ' 1 ' refers to January,..., and '12' refers to December. 
Nikurashin et al. (2013) and Abe and Nakamura (2013) also found that diapycnal mixing is enhanced in regions where there is rough topography and where lee waves break. Here, we do not explicitly resolve sub-mesoscale motions, but it is reassuring that the diapycnal mixing is enhanced, leading to a reduction in abyssal stratification, when wave drag is inserted into the model.

The differences between the offline wave drag dissipation rates calculated from the average velocities in the bottom $500 \mathrm{~m}$ in the simulation without wave drag and those in the simulation with wave drag are given in Fig. 13. The offline dissipation rates are estimated using WOA stratification in Fig. 13a and using the stratification from each of the two model simulations in Fig. 13b. The relatively similar appearances of Fig. 13a and b suggest that the velocities have a stronger impact on wave drag than the stratification. The velocities in the bottom $500 \mathrm{~m}$ are altered in the simulation with wave drag such that the globally integrated dissipation rates associated with wave drag are cut by more than half. Some noteworthy locations where the dissipation rates are largely reduced include the Arctic Ocean, the Indian Sector of the Southern Ocean, and in equatorial regions. However, the stratification and velocities in the bottom $500 \mathrm{~m}$ are altered in the simulation with wave drag such that the globally integrated wave drag dissipation rates are only cut in half. Regions where the stratification has a relatively strong impact include the Arctic Ocean, the Subpolar North Atlantic, and in the wake of Drake Passage, as evidenced by the differences between Fig. 13a and b.

\subsection{Total mechanical energy budget analysis}

The seasonality of the terms in our total mechanical energy budget, (22), are shown in Fig. 14. The largest terms are the source and sink terms from the momentum equations, which are highly correlated and balance each other out to within $23.7 \%(2.31 \%)$ in the simulation with (without) wave drag. The next most dominant terms are the advective flux of potential energy and partial time derivative of potential energy, followed by the conversion from internal energy to potential energy, the latter of which is highly correlated with the source and sink terms from the momentum equations. The buoyancy diffusion term is of the same order of magnitude as the conversion from internal energy to potential energy term. The pressure work term is relatively small, which is

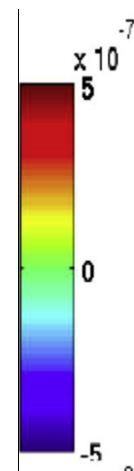

$-3$

(a) Pressure work [ $\left.\mathrm{W} \mathrm{m}^{-2}\right]$

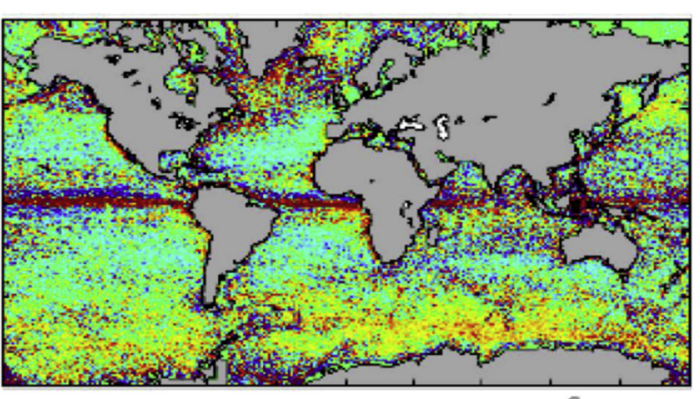

(c) IE to PE conversion [W $\mathrm{m}^{-2}$ ]
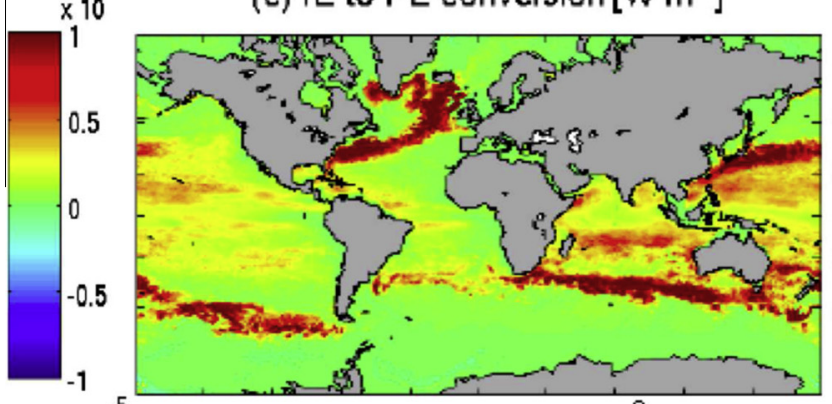

(e) $K E$ advection $\left[\mathrm{W} \mathrm{m}^{-2}\right]$

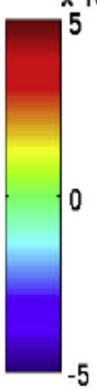

$\times 10$

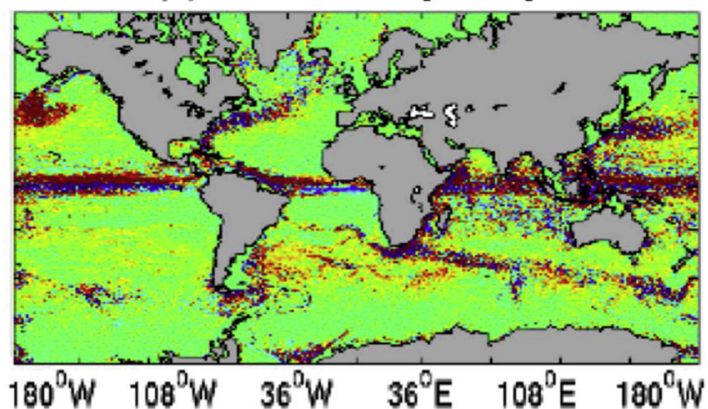

(b) Buoyancy diffusion $\left[\mathrm{W} \mathrm{m}^{-2}\right]$

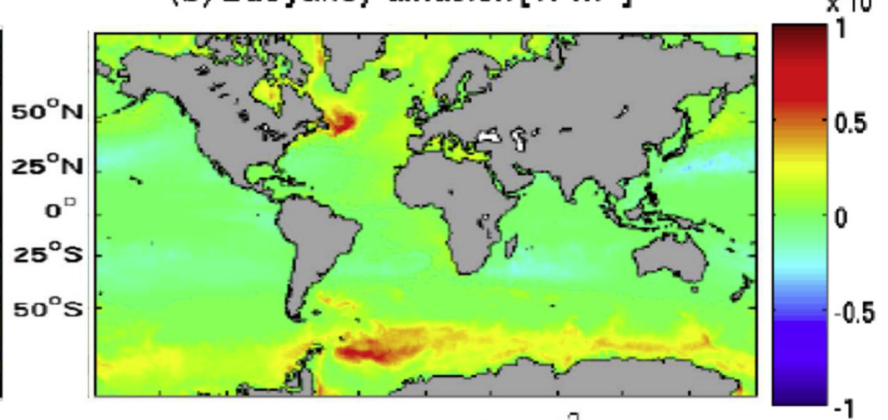

(d) PE advection $\left[\mathrm{W} \mathrm{m}^{-2}\right]$
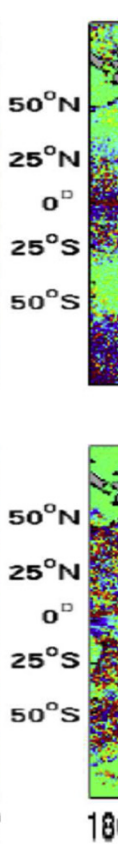

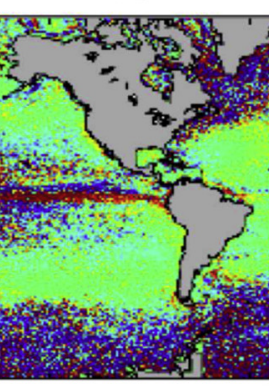

(f) $P E$ time $\left[\mathrm{W} \mathrm{m} \mathrm{m}^{-2}\right]$

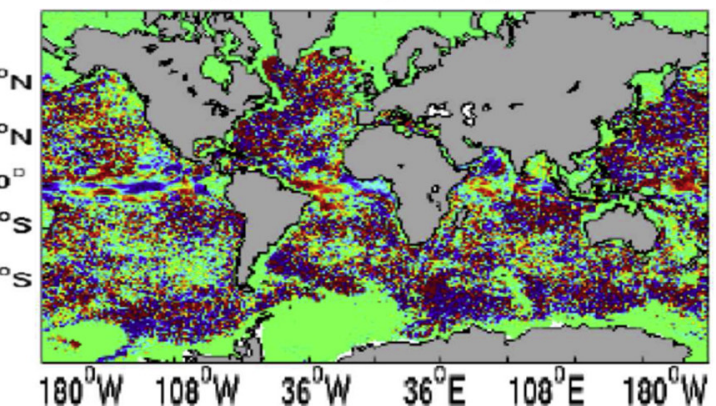

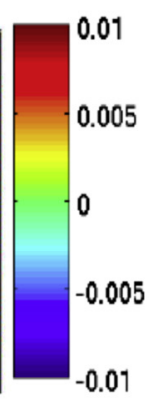

$-0.01$

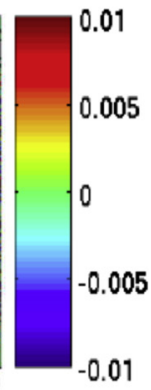

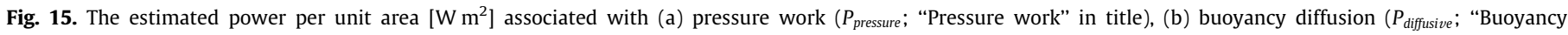

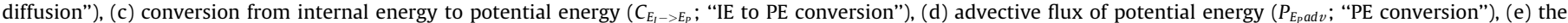

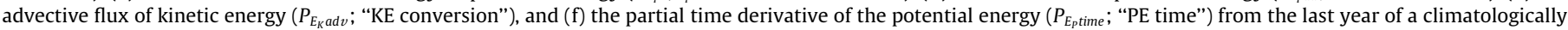
forced spin-up of HYCOM with wave drag. 
Table 3

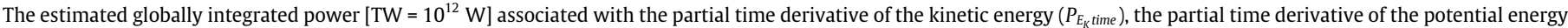

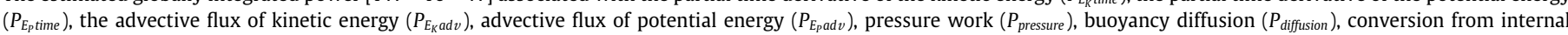
energy to potential energy $\left(C_{E_{1}->E_{p}}\right)$, input $\left(P_{\text {input }}\right)$, and output $\left(P_{\text {output }}\right)$ averaged over the final year of the spin-up of HYCOM with wave drag.

\begin{tabular}{|c|c|c|c|c|c|c|c|c|}
\hline$P_{E_{K} \text { time }}$ & $P_{E_{p} \text { time }}$ & $P_{E_{K} a d v}$ & $P_{E_{p} a d v}$ & $P_{\text {pressure }}$ & $P_{\text {diffusion }}$ & $C_{E_{I}->E_{p}}$ & $P_{\text {input }}$ & $P_{\text {output }}$ \\
\hline 0.0126 & 0.146 & 0.00284 & -0.174 & $2.81 \times 10^{-6}$ & 0.0309 & 0.0865 & 0.868 & 1.06 \\
\hline
\end{tabular}

expected because the ocean's mean sea surface height would otherwise be significantly varying along with the Earth's geoid. The magnitude of the sum of the partial time derivative of kinetic energy and potential energy terms suggests that there is non-steady-state behavior in the model. The degree to which the model is not in steady-state can further be diagnosed by looking at Fig. 1, which suggests that the time derivative of the kinetic energy is still changing but by very little.

With the exception of the partial time derivative of the kinetic energy term, the spatial distributions of each of the non-input/output terms in (22) are given in Fig. 15. The advective flux of potential energy and kinetic energy terms (Fig. 15d and e), pressure work term (Fig. 15a), and partial time derivative of potential energy term (Fig. 15f) each take on their largest values near the equator. The conversion from internal energy to potential energy term (Fig. 15c) takes on its largest values near the intensified jets. The buoyancy diffusion term (Fig. 15b) takes on its largest values just equatorward of where deep convection occurs. The sum of the conversion from internal energy to potential energy and buoyancy diffusion terms is very similar in spatial distribution to the buoyancy flux (not shown) because each of these terms is closely related to the stratification via a vertical density gradient. The sum of the advective flux of potential energy term (Fig. 15d) and and the partial time derivative of potential energy term (Fig. 15f) roughly balances the sum of the rest of the terms.

Our total mechanical energy budget, (22), is approximately balanced (Table 3 ) with $a<10 \%$ imbalance relative to the sum of the dissipators. There are several potential reasons for the imbalance between the sum of the partial time derivative and advective flux terms $(-0.0126 \mathrm{TW})$ and the sum of the pressure work, buoyancy diffusion, internal to potential energy conversion, input, and output terms $(-0.0746 \mathrm{TW})$.

(1) While the Robert-Asselin time filter suppresses a spurious time-splitting computational mode associated with the use of the leap-frog time-stepping scheme (Griffies et al., 2000), it is an unmeasured source of dissipation. An associated imbalance in surface forcing gradually leads to a small violation of tracer (e.g., heat) conservation over time (Leclair and Madec, 2009). These factors could account for some of the disagreement in our total mechanical energy budget. The dissipation due to the Robert-Asselin filter in a one-layer $\mathrm{M}_{2}$ tide run of HYCOM was quantified by doubling the filter weights. It was found that the dissipation is less than $0.05 \mathrm{TW}$, out of a total $M_{2}$ dissipation rate of about $2.4 \mathrm{TW}$ (not shown).

(2) We used centered differences for all of the spatial derivatives and our model frequently implements an interpolation scheme between density and z-coordinates. These numerical errors are propagated and may be significant in a non-uniformly spaced coordinate system such as that of a time-varying isopycnic coordinate model with a tripole grid.

\section{(a) Info. tensor $(1,1)$ GAM residuals}

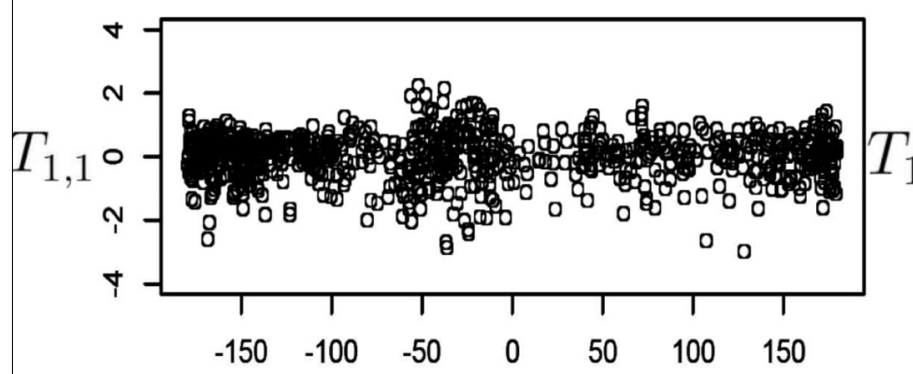

(c) Info. tensor $(2,1)$ GAM residuals

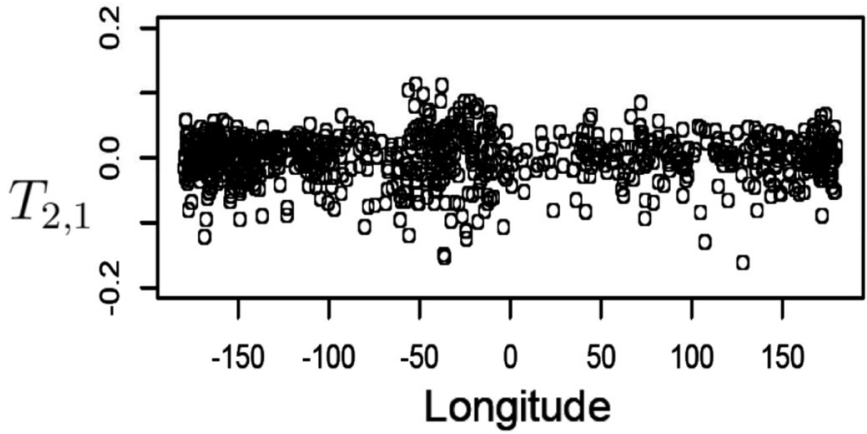

(b) Info. tensor $(1,2)$ GAM residuals

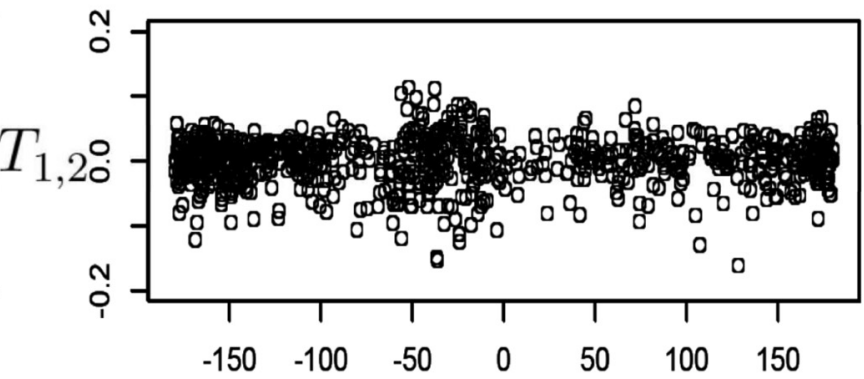

(d) Info. tensor $(2,2)$ GAM residuals

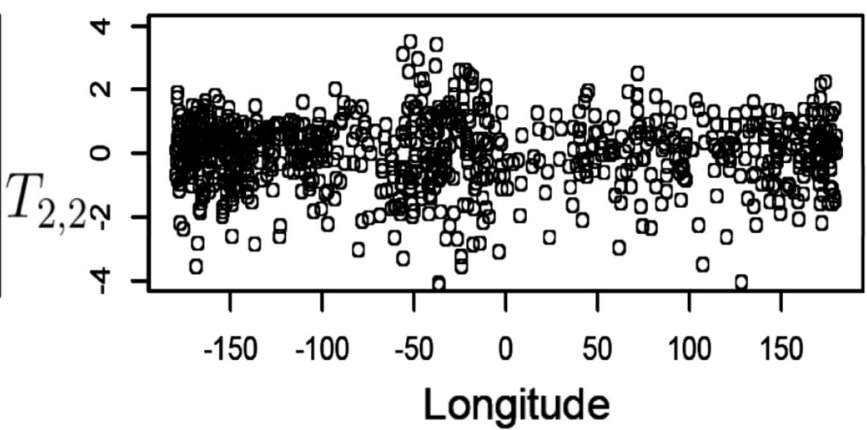

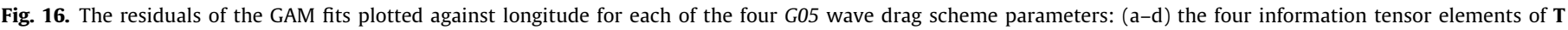
$\left[\mathrm{kg} \mathrm{m}^{-2} \mathrm{~s}^{-1}\right]$. 


\section{(a) $(1,1)$ component of info. tensor (b) $10 \times(1,2)$ component of info. tensor}
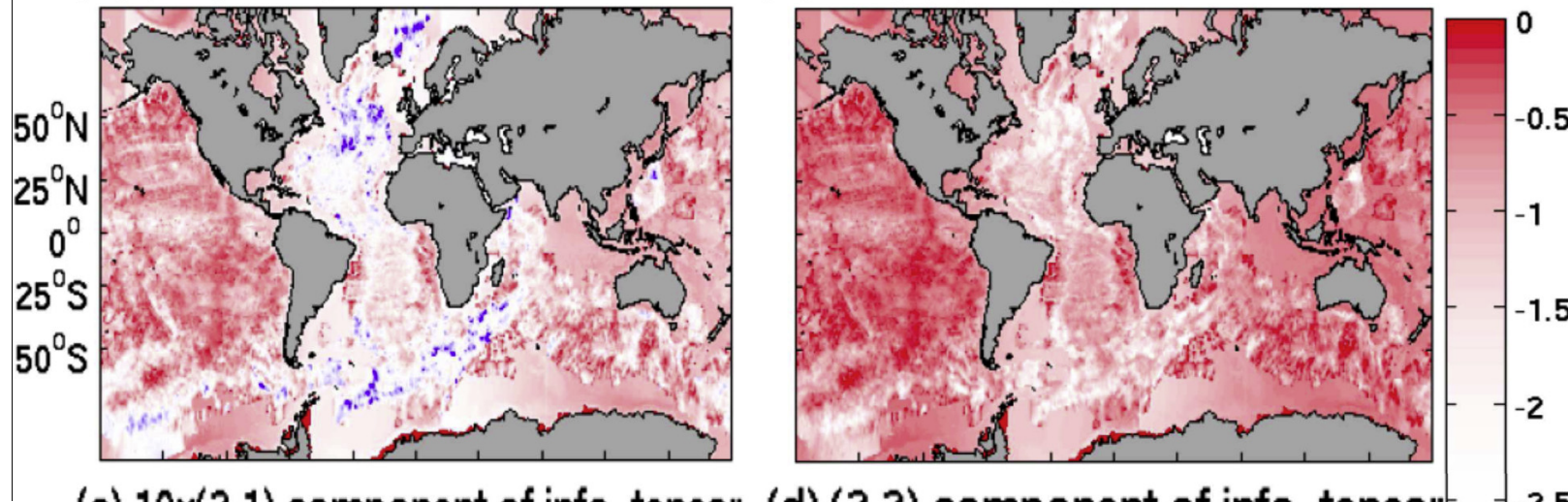

(c) $10 \times(2,1)$ component of info. tensor
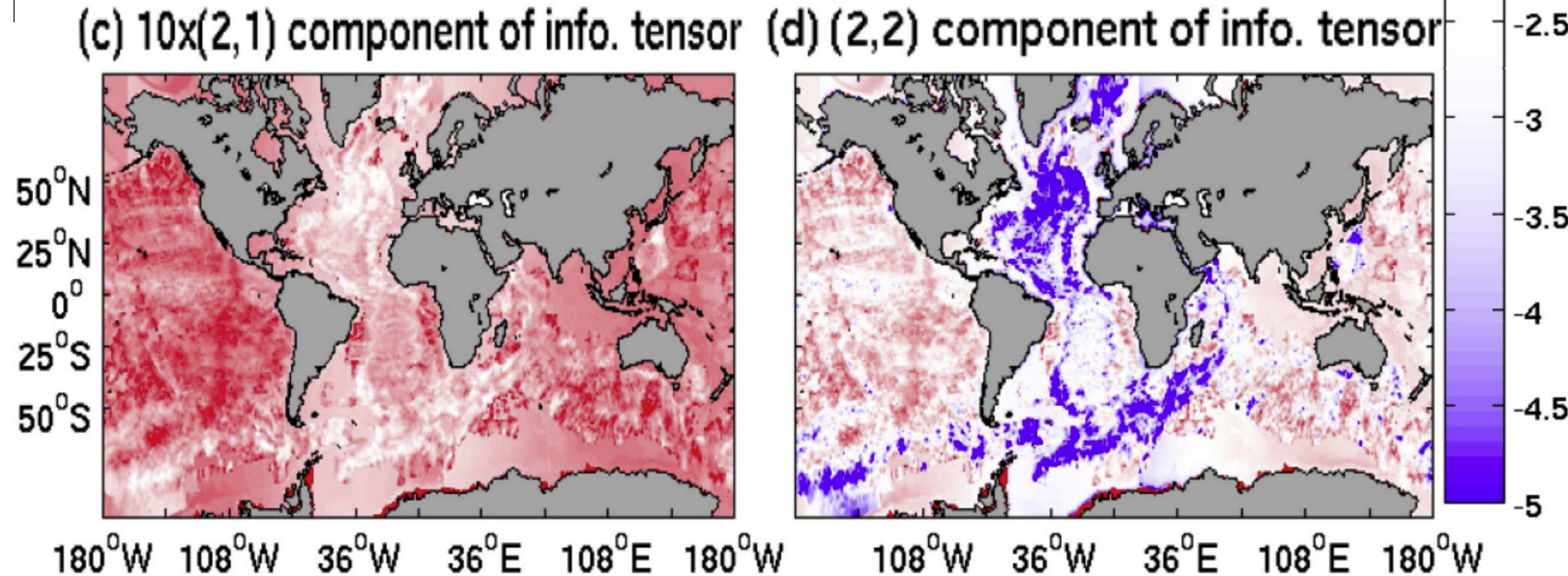

$180^{\circ} \mathrm{W} \quad 108^{\circ} \mathrm{W} \quad 36^{\circ} \mathrm{W} \quad 36^{\circ} \mathrm{E} \quad 108^{\circ} \mathrm{E} \quad 180^{\circ} \mathrm{W}$

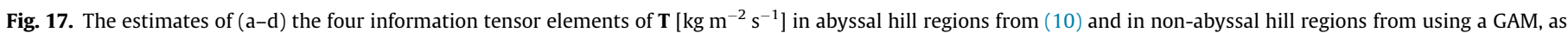
described in Appendix B.

(3) HYCOM consistently makes the approximation that the nonsteric sea surface height (i.e., bottom pressure anomaly) is a small fraction of the total depth. We are using the same approximation in the total mechanical energy budget, which might be more accurate if we instead used the total depth including the non-steric sea surface height.

(4) We may need to explicitly account for the compressibility of water from thermobaricity. However, this term is a global integral over $p(\partial \rho / \partial z) / \rho$, which should be small because this is of a comparable order of magnitude as the buoyancy diffusion term we have computed here.

(5) The power associated with the along-isopycnal diffusion of buoyancy may be non-negligible. Using typical values of each of the variables in $g \vec{\nabla} \cdot(\kappa z \vec{\nabla} \rho)$, we estimate that the diapycnal contribution to the diffusion of buoyancy term is of the same order of magnitude as the along-isopycnal contribution to the diffusion of buoyancy term. This could potentially account for our $<10 \%$ energy budget imbalance and is of the correct sign.

\section{Conclusions}

The net dissipative and input terms in a total mechanical energy budget have been presented here for an eddying global ocean model with and without an inline parameterization of topographic internal lee wave drag. Topographic internal lee wave drag and quadratic bottom boundary layer drag each contribute a significant amount of the total energy dissipation in the model. Horizontal and vertical eddy viscosity are both responsible for energy dissipation rates greater than those associated with bottom drag, but less than those associated with wave drag. Most of the energy dissipation associated with horizontal viscosity occurs at the margins of the intensified currents of the ocean. The energy dissipation associated with vertical viscosity takes place primarily within the mixed layer. The energy dissipation rate by bottom drag is reduced to a greater extent upon addition of wave drag to the model than is the energy dissipation rate by either of the viscosity terms. However, utilization of a boosted bottom drag cannot serve as a substitute for a wave drag parameterization. Energy dissipation by wave drag more than compensates for the decrease in energy dissipation rates via bottom drag, vertical viscosity, and horizontal viscosity.

Our inline energy budget analysis is preceded by our own offline analysis, which is much faster to perform. In contrast to previous offline estimates of globally integrated dissipation by lee wave drag (Nikurashin and Ferrari, 2011; Scott et al., 2011), our own offline estimates have used two different wave drag schemes for comparison. Offline estimates show that the G05 wave drag scheme yields comparable spatial dissipation maps and comparable globally integrated dissipation rates to the $B 75$ scheme in regions where there are abyssal hills. Our offline globally integrated dissipation rates are closer to those of Scott et al. (2011) than to those of Nikurashin and Ferrari (2011). When extended to regions without abyssal hills, the offline globally integrated dissipation rates are approximately tripled. Another important contrast between our study and previous studies is that we estimate the dissipation rates inline as the model runs as well as offline. The extra roughness we choose to insert in regions where there are no abyssal hills doubles the inline globally integrated dissipation rates. However, the inline feedback that reduces the abyssal velocities and stratification lowers the globally integrated dissipation rates. In the end, the two factors 
roughly cancel each other so that our inline estimates of globally integrated dissipation rates by wave drag are comparable to global offline estimates made in previous studies (Nikurashin and Ferrari, 2011; Scott et al., 2011). The equator is the region with the largest absolute reduction in energy dissipation rates due to wave drag taking place in inline versus offline estimates. The regions having the largest percent reductions in wave drag energy dissipation rates in inline versus offline estimates are generally regions where dissipation is relatively small (mostly non-abyssal hill regions).

We have evaluated an approximately balanced total mechanical energy budget. There are a number of confounding factors that could be responsible for the imbalance of our total mechanical energy budget. It is possible that the compressibility effects are significant and/or that the along-isopycnal contributions to the diffusion of buoyancy term are important. Errors in the numerical methods, and approximations used in the model, may contribute to the total mechanical energy budget's imbalance as well.

We recommend several improvements to our implementation of wave drag. First, the range of relevant wavenumbers for the internal waves to not be evanescent can be different for different regions of the ocean, in contrast to the fixed range we specified by (6). Second, a more physically motivated estimation of the roughness parameters in non-abyssal hill regions, taken from a formulation that characterizes the statistical features with a power spectrum, would be an improvement over the machine learning algorithm we used here (Appendix B). Third, the form of the wave drag parameterization in the momentum equations could be improved upon. The full tensor wave drag in (11) is preferable to the reduced scalar form in (14) used here. Fourth, the assumption of $100 \%$ local dissipation in the bottom $500 \mathrm{~m}$ can be relaxed by using the depth-dependent momentum deposition procedure developed in G05. Fifth, it should be investigated how to implement wave drag in a subset of regions (e.g., only in regions where the seafloor is deeper than $500 \mathrm{~m}$, only in abyssal hill regions, only where $f<N$, or only where topographic slopes are not supercritical) while maintaining numerical stability. Sixth, the diapycnal diffusivity could be adjusted (e.g., Polzin, 2009) according to the energy dissipation arising from the wave drag scheme in addition to the indirect effects on the diapycnal diffusivity arising from changes in the vertical shear which then activate changes through KPP. Lastly, the implementation of the G05 scheme would benefit from the development of a procedure to constrain all of its free parameters that are directly related to the magnitude of the wave drag. Alternatively, a different wave drag scheme could be used, but different problems, in addition to some of the aforementioned ones, would then arise.

Here, we have focused on the impact of parameterized topographic internal lee wave drag on model energetics. We still need to examine whether the inclusion of this parameterization improves the performance of HYCOM relative to various in situ and satellite observations. Furthermore, we need to examine how the role of wave drag in the energy budget changes as the horizontal resolution of the model is increased. These currently unresolved issues will be pursued in a follow-up study.

\section{Acknowledgments}

The authors of this study would like to thank two anonymous reviewers for their helpful suggestions which led to a much improved manuscript. D.S. Trossman and B.K. Arbic gratefully acknowledge support from National Science Foundation (NSF) Grant OCE-09607820 and Office of Naval Research (ONR) Grant N00014-11-1-0487. B.K. Arbic and J.A. Goff gratefully acknowledge support from ONR Grants N00014-07-1-0392 and N00014-09-11003. S.R. Jayne gratefully acknowledges support from NSF Grant OCE-0960756. A.J. Wallcraft and E.J. Metzger gratefully acknowl- edge the "6.2 Eddy Resolving Global Ocean Prediction Including Tides" project sponsored by the Office of Naval Research under program element 0602435N. D.S. Trossman and B.K. Arbic would like to thank Eric Kunze and Kurt Polzin for insightful discussions regarding observations in regions where lee wave drag is likely to occur, and Robert Hallberg and Stephen Griffies for useful discussions about the total mechanical energy budget of an ocean model. Grants of computer time were provided by the Department of Defense (DoD) High Performance Computing Modernization Program. The simulations were performed on the SGI Altix Ice at the US Army Engineer Research and Development Center DoD Supercomputing Resource Center in Vicksburg, MS. This is Naval Research Laboratory contribution NRL/JA/7320-12-1208 and has been approved for public release.

\section{Appendix A. Wave Drag Parameter Estimation}

In order to implement the wave drag scheme of Garner (2005; G05 hereafter), we need to estimate two parameters that are assumed to be spatially homogeneous, $\gamma$ and $\epsilon$, and one parameter that we allow to vary in space, $\beta$. The first of these relates the maximum height of topographic features to their horizontal scale and the second determines the number density of features as a function of their maximum height. Using the same method (linear regression using functions of the velocity potential for linear mountain waves) described in the Appendix of G05 but applied to the synthetic topography described in Section 3.2 generated on a 2-min grid, we find that $\gamma=0.36$, which is close to the value of 0.4 in G05. The other uniform parameter, $\epsilon$, is estimated as the leastsquares slope of $\log (n(h))$ versus $\log (\Delta h)$, where $n(h)$ is the number of topographic height maxima in the interval $\Delta h$ sampled from $10^{\circ} \times 10^{\circ}$ boxes. We find that $\epsilon=0.02$. The examples in G05 assumed $\epsilon=0$.

The third parameter, $\beta$, establishes the shape of the vertical cross-section of the hills for the dimensional analysis. We keep the mean value of $\beta=0.5$ used by $G 05$ (midway between a triangular and flat summit) and focus on the variability about the mean. Preliminary model runs suggest that the standard deviation should be on the order of 0.25 . $^{12}$ We impose this variability by first examining the distribution of $\Delta \beta_{i, j}=\left|h_{x_{i+1, j}}-h_{x_{i-1, j}}\right|+\left|h_{y_{i, j+1}}-h_{y_{i, j-1}}\right|$ where $h_{x_{i, j}}=h_{i+1, j}-h_{i-1, j}, h_{y_{i, j}}=h_{i, j+1}-h_{i, j-1}$, and $h_{i, j}$ is the gridded high-resolution topography with $i$ and $j$ denoting longitude and latitude indices. $\Delta \beta$ is a measure of the steepness of the local terrain. Then we set

$\beta_{i, j}=0.4+0.0006 \Delta \beta_{i, j}$

which has the desired mean and standard deviation.

There are other tunable parameters associated with the wave drag scheme of G05. For example, $a_{0}$ is a coefficient for the propagating component of wave drag and $a_{1}$ is a coefficient for the non-propagating component of wave drag. We follow G05 and constrain these remaining parameters to satisfy the relationship, $\left(a_{1} / a_{0}\right)=\left(\widetilde{H}_{c r i t} / \widetilde{H}_{c r i t, r}\right)$ (where $\widetilde{H}_{c r i t}$ and $\widetilde{H}_{\text {crit, },}$ are the non-dimensionalized critical height and its reference). G05 assumed that $\widetilde{H}_{\text {crit }, r}=1 / 9$ to yield a maximum total drag of about $2 D^{*}$, where $D^{*}$ is the base flux of momentum with $\widetilde{H}_{\text {crit }}=0.7$ and $a_{0}=1$. We follow suit here.

\footnotetext{
12 Using the WOA bottom stratification and bottom velocities from June and December of the final year of the HYCOM simulation without wave drag, we found that using values for $\beta$ anywhere between 0 and 1 yielded energy dissipation rates to be well within a factor of 2 of those from Scott et al., 2011.
} 


\section{Appendix B. Extending inputs for the G05 scheme to regions without abyssal hills}

The Goff and Arbic (2010) and Goff (2010) fields are only defined in regions where abyssal hills are defined according to Goff and Jordan (1988). However, the numerics of HYCOM are unstable if we apply large wave drag at one grid point and no wave drag at an adjacent grid point. Also, there exists observational evidence (St. Laurent et al., 2012) that the roughness in some non-abyssal hill regions may be sufficient to generate lee waves. Therefore, we pursue a method to estimate $\mathbf{T}$ such that each element of $\mathbf{T}$ smoothly varies toward zero away from the abyssal hills. We use a machinelearning algorithm called a Generalized Additive Model [GAM; using the mgc $v$ package's bam in R (Wood, 2006)] with the functional dependence,

$T_{i, j}=f_{1, i, j}(h, \beta)+f_{2, i, j}(h, \lambda)+f_{3, i, j}(\beta, \lambda)$.

Here, $h$ is the root mean square topographic height; $\beta$ is from (A.1); $\lambda$ is longitude; and $f_{n, i, j}$ for $n=1,2,3, i=1,2$, and $j=1,2$ are smooth but nonlinear functions determined by the best fit subject to a condition that the integral over the square of their second derivative, a measure of "wiggliness" as described in another context by Trossman et al. (2011), is not too large. Here, $T_{1,1}, T_{1,2}, T_{2,1}$, and $T_{2,2}$ are elements of $\mathbf{T}$. The estimates of each $T_{i, j}$ are nearly insensitive to latitudinal dependencies because topographic roughness varies more with longitude than latitude. Eight GAMs are trained where there are abyssal hills from Goff and Arbic (2010). Two GAMs are trained for each of the four $T_{i, j}$ : one for regions east of the Prime Meridian and one for regions west of the Prime Meridian. If each $T_{i, j}$ is estimated using only one GAM, then the values of each $T_{i, j}$ are larger at every location where there are no abyssal hills. Each GAM, with residuals shown to be uniformly scattered as a function of longitude in Fig. 16, is used to predict what the values of the four $T_{i, j}$ are everywhere outside of the abyssal hill regions. Fig. 16 also shows that there is no systematic bias in our GAM-based estimates and that the standard error always stays less than a few tens of percent of the field.

GAMs are designed to be able to predict outside of their training data, which we do here. We tend to predict each $T_{i, j}$ outside of the abyssal hill regions using values of at least two of $\beta, h$, and $\lambda$ found in regions where there are abyssal hills. For example, both inside and outside of the abyssal hill regions we can have small values for $\beta$ at a given longitude. However, the magnitude of $h$ can be different in abyssal hill regions from $h$ in non-abyssal hill regions along the same longitude near a coastal margin. The regions where we predict each $T_{i, j}$ tend to have small $\beta$ and widely varying $h$ for each $\lambda$.

Lastly, we smooth the resulting horizontal gradients of each of the $T_{i, j}$ along the boundaries of the abyssal hill regions. To do this, we apply a Gaussian Blackman filter (with fspecial, fwind2, and filter2 in Matlab) using 31 grid points everywhere, zeroing out the normalized frequencies greater than 0.5 and less than 0.1 . The original values of each of the $T_{i, j}$ in regions where there are abyssal hills are reassigned so that each of the $T_{i, j}$ is left unaltered by the Blackman filter in regions where there are abyssal hills.

Each element of the information tensor, T, is shown in Fig. 17. As expected, the diagonal components of $\mathbf{T}$ (Fig. 17a and d) are of the same order of magnitude, and the off-diagonal components of $\mathbf{T}$ (Fig. 17b and c) are an order of magnitude less than the diagonal components. In general, the values of $\mathbf{T}$ are larger in the Atlantic than in the Pacific. The GAMs find that there are several topographic features that could be involved in lee wave generation in the North Atlantic Ocean (i.e., in the areas surrounding Green- land and Iceland). The values away from the abyssal hills in Fig. 17 decay to zero within a few degrees except around Greenland and Iceland, where there may be enough roughness to generate internal lee waves.

\section{References}

Abe, S., Nakamura, T., 2013. Processes of breaking of large-amplitude unsteady lee waves leading to turbulence, J. Geophys. Res. Oceans 118, 1-16.

Aiki, H., Richards, K.J., Sakuma, H., 2011. Maintenance of the mean kinetic energy in the global ocean by the barotropic and baroclinic energy routes: the roles of JEBAR and Ekman dynamics. Ocean Dyn. 61, 675-700.

Alford, M.H., 2003. Improved global maps and 54-year history of wind-work on ocean inertial motions. Geophys. Res. Lett. 30, 1424-1427.

Arbic, B.K., Flierl, G.R., 2004. Baroclinically unstable geostrophic turbulence in the limits of strong and weak bottom ekman friction: Application to midocean eddies. J. Phys. Oceanogr. 34, 2257-2273.

Arbic, B.K., Garner, S.T., Hallberg, R.W., Simmons, H.L., 2004. The accuracy of surface elevations in forward global barotropic and baroclinic tide models. Deep-Sea Res.-II 51, 3069-3101.

Arbic, B.K., Scott, R.B., 2008. On quadratic bottom drag, geostrophic turbulence, and ocenic mesoscale eddies. J. Phys. Oceanogr. 38, 84-103.

Arbic, B.K., Shriver, J.F., Hogan, P.J., Hurlburt, H.E., McClean, J.L., Metzger, E.J., Scott, R.B., Sen, A., Smedstad, O.M., Wallcraft, A.J., 2009. Estimates of bottom flows and bottom boundary layer dissipation of the oceanic general circulation from global high-resolution models. J. Geophys. Res. 114, C02024.

Arbic, B.K., Wallcraft, A.J., Metzger, E.J., 2010. Concurrent simulation of the eddying general circulation and tides in a global ocean model. Ocean Modell. 32, 175187

Beaird, N.L., Fer, I., Rhines, P.B., Eriksen, C.C., 2012. Dissipation Rate and Vertical Mixing Inferred from Seagliders: An Application to the Nordic Seas Overflows. J. Phys. Oceanogr. 42, 2268-2282.

Bell Jr., T.H., 1975. Topographically generated internal waves in the open ocean. J. Geophys. Res. 80, 320-327.

Bleck, R., 2002. An oceanic general circulation model framed in hybrid isopycnicCartesian coordinates. Ocean Modell. 37, 55-88.

Chassignet, E.P., Smith, L.T., Halliwell, G.R., Bleck, R., 2003. North Atlantic simulations with the Hybrid Coordinate Ocean Model (HYCOM): Impact of the vertical coordinate choice, reference pressure, and thermobaricity. J. Phys. Oceanogr. 33, 2504-2526.

Dewar, W.K., Hogg, A.M., 2010. Topographic inviscid dissipation of balanced flow. Ocean Modell. 32, 1-13.

Dewar, W.K., Berloff, P., Hogg, A.M., 2011. Submesoscale generation by boundaries. J. Mar. Res. 69, 501-522.

Egbert, G.D., Ray, R.D., Bills, B.G., 2004. Numerical modeling of the global semidiurnal tide in the present day and in the Last Glacial Maximum. J. Geophys. Res. 109, C03003.

Ferrari, R., Wunsch, C., 2010. The distribution of eddy kinetic and potential energies in the global ocean. Tellus A 62, 92-108.

Fox-Kemper, B., Pedlosky, J., 2004. Wind-driven barotropic gyre I: Circulation control by eddy vorticity fluxes to an enhanced removal region. J. Mar. Res. 62, 169-193.

Fox-Kemper, B., Menemenlis, D., 2008. Can large eddy simulation techniques improve mesoscale rich ocean models? In: Hecht, M., Hasumi, H. (Eds.), Ocean Modelling in an Eddying Regime, vol. 177. AGU Geophysical Monograph Series, pp. 319-338.

Furuichi, N., Hibiya, T., Niwa, Y., 2008. Model-predicted distribution of windinduced internal wave energy in the worlds oceans. J. Geophys. Res.-Oceans 113, C09034.

Garner, S.T., 2005. A topographic drag closure built on an analytical base flux. J. Atmos. Sci. 62, 2302-2315.

Gnanadesikan, A., Slater, R.D., Swathi, P.S., Vallis, G.K., 2005. The energetics of ocean heat transport. J. Clim. 18, 2604-2616.

Goff, J.A., Jordan, T.H., 1988. Stochastic modeling of seafloor morphology inversion of sea beam data for second-order statistics. J. Geophys. Res. 93, 13589-13608.

Goff, J.A., 2010. Global prediction of abyssal hill root-mean-square heights from small-scale altimetric gravity variability. J. Geophys. Res. 115, B12104.

Goff, J.A., Arbic, B.K., 2010. Global prediction of abyssal hill roughness statistics for use in ocean models from digital maps of paleo-spreading rate, paleo-ridge orientation, and sediment thickness. Ocean Modell. 32, 36-43.

Griffies, S.M., 2004. Fundamentals of Ocean Climate Models. Princeton University Press, $518 \mathrm{pp}$

Griffies, S.M., Böning, C., Bryan, F.O., Chassignet, E.P., Gerdes, R., Hasumi, H., Hirst, A. Treguier, A.-M., Webb, D., 2000. Developments in ocean climate modelling. Ocean Modell. 2, 123-192.

Hallberg, R., 2005. A thermobaric instability of Lagrangian vertical coordinate ocean models. Ocean Modell. 8, 279-300.

Halliwell, G.R., 2004. Evaluation of vertical coordinate and vertical mixing algorithms in the HYbrid-Coordinate Ocean Model (HYCOM). Ocean Modell. 7. $285-322$. 
Hogg, A., Dijkstra, H., Saenz, J., 2013. The Energetics of a Collapsing Meridional Overturning Circulation. J. Phys. Oceanogr. 43, 1512-1524.

Jayne, S.R., St. Laurent, L.C., 2001. Parameterizing tidal dissipation over rough topography. Geophys. Res. Lett. 28, 811-814.

Jochum, M., Danabasoglu, G., Holland, M., Kwon, Y.-O., Large, W.G., 2008. Ocean viscosity and climate. J. Geophys. Res. 113, C06017.

Kallberg, P., Simmons, A., Uppala, S., Fuentes, M., 2004. The ERA-40 Archive, ERA-40 Project Report Series No. 17, ECMWF.

Firing, Kunze E.E., Hummon, J.M., Chereskin, T.K., Thurnherr, A.M., 2006. Global abyssal mixing inferred from lowered ADCP shear and CTD strain profiles. J. Phys. Oceanogr. 36 (8), 1553-1576.

Kunze, E., MacKay, C., McPhee-Shaw, E.E., Morrice, K., Girton, J.B., Terker, S.R., 2012. Turbulent mixing and exchange with interior waters on sloping boundaries. J. Phys. Oceanogr. 42, 910-927.

Large, W.G., McWilliams, J.C., Doney, S.C., 1994. Oceanic vertical mixing: a review and a model with a nonlocal boundary layer parameterization. Rev. Geophys. 32, 363-403.

Leclair, M., Madec, G., 2009. A conservative leapfrog time stepping method. Ocean Modell. 30, 88-94.

Ledwell, J.R., Montgomery, E.T., Polzin, K.L., St. Laurent, L.C., Schmitt, R.W., Toole, J.M., 2000. Evidence for enhanced mixing over rough topography in the abyssal ocean. Nature 403, 182-189.

Marshall, D.P., Naveira-Garabato, A.C., 2008. A conjecture on the role of bottomenhanced diapycnal mixing in the parameterization of geostrophic eddies. J. Phys. Oceanogr. 38, 1607-1613.

McDougall, T.J., Barker, P.M., 2011. Getting started with TEOS-10 and the Gibbs Seawater (GSW) Oceanographic Tollbox, 28 pp., SCOR/IAPSO WG127, ISBN 9780-646-55621-5.

Müller, P., McWilliams, J.C., Molemaker, M.J., 2005. Routes to dissipation in the ocean: The 2D/3D turbulence conumdrum. In: Baumert, H.Z., Simpson, J., Sündermann, J. (Eds.), Marine Turbulence. Cambridge University Press, Cambridge, UK, pp. 397-405.

Munk, W., Wunsch, C., 1998. Abyssal recipes II: Energetics of tidal and wind mixing. Deep Sea Res. I 45, 1977-2010.

Murray, R.J., 1996. Explicit generation of orthogonal grids for ocean models. J. Comput. Phys. 126, 251-273.

Naveira-Garabato, A.C., Polzin, K.L., King, B.A., Heywood, K.J., Visbeck, M., 2004. Widespread intense turbulent mixing in the Southern Ocean. Science 303, 210 213.

Nikurashin, M., 2008. Radiation and dissipation of internal waves generated by geostrophic motions impinging on small-scale topography, PhD thesis. MIT/ Woods Hole Ocean. Inst. Joint Program, Cambridge, MA.

Nikurashin, M., Ferrari, R., 2011. Global energy conversion rate from geostrophic flows into internal lee waves in the deep ocean. Geophys. Res. Lett. 38, L08610.

Nikurashin, M., Vallis, G.K., Adcroft, A., 2013. Routes to energy dissipation for geostrophic flows in the Southern Ocean. Nature Geosci. 6, 48-51.

Oort, A.H., Anderson, L.A., Peixoto, J.P., 1994. Estimates of the energy cycle of the oceans. J. Geophys. Res.-Oceans 99, 7665-7688.

Polzin, K.L., Toole, J.M., Ledwell, J.R., Schmitt, R.W., 1997. Spatial variability of turbulent mixing in the Abyssal Ocean. Science 276, 93-96.

Polzin, K.L., 2008. Mesoscale eddy-internal wave coupling. Part I: Symmetry, wave capture, and results from the mid-ocean dynamics experiment. J. Phys. Oceanogr. 38, 2556-2574.

Polzin, K.L., 2009. An abyssal recipe. Ocean Modell. 30, 298-309.

Qiao, F., Huang, C.J., 2012. Comparison between vertical shear mixing and surface wave-induced mixing in the extratropical ocean. J. Geophys. Res.-Oceans 117, C00J16.

Rosmond, T.E., Teixeira, J., Peng, M., Hogan, T.F., Pauley, R., 2002. Navy Operational Global Atmospheric Prediction System (NOGAPS): Forcing for ocean models. Oceanography 15, 99-108.

Saenko, O.A., Zhai, X., Merryfield, W.J., Lee, W.G., 2012. The combined effect of tidally and eddy-driven diapycnal mixing on the large-scale ocean circulation. J. Phys. Oceanogr. 42, 526-538.

Saenz, J.A., Hogg, A.M., Hughes, G.O., Griffiths, R.W., 2012. Mechanical power input from buoyancy and wind to the circulation in an ocean model. Geophys. Res. Lett. 39, L13605.

Scott, R.B., Xu, Y., 2009. An update on the wind power input to the surface geostrophic flow of the World Ocean. Deep-Sea Res. I 56, 295-304.

Scott, R.B., Goff, J.A., Naveira-Garabato, A.C., Nurser, A.J.G., 2011. Global rate and spectral characteristics of internal gravity wave generation by geostrophic flow over topography. J. Geophys. Res. 116, C09029.

Sen, A., Scott, R.B., Arbic, B.K., 2008. Global energy dissipation rate of deep-ocean low-frequency flows by quadratic bottom boundary layer drag: Computations from current-meter data. Geophys. Res. Lett. 35, L09606.
Shriver, J.F., Arbic, B.K., Richman, J.G., Ray, R.D., Metzger, E.J., Wallcraft, A.J., Timko, P.G., 2012. An evaluation of the barotropic and internal tides in a high resolution global ocean circulation model. J. Geophys. Res.-Oceans 117, C10024.

Shu, Q., Qiao, F., Song, Z., Xia, C., Yang Y., 2011. Improvement of MOM4 by including surface wave-induced vertical mixing. Ocean Modell. 40, 42-51.

Smagorinsky, J., 1993. Large eddy simulation of complex engineering and geophysical flows. In: Galperin, B., Orszag, S.A. (Eds.), Evolution of Physical Oceanography. Cambridge University Press, pp. 3-36.

St. Laurent, L.C., Toole, J.M., Schmitt, R.W., 2001. Buoyancy forcing by turbulence above rough topography in the abyssal Brazil Basin. J. Phys. Oceanogr. 31, 34763495.

St. Laurent, L.C., Simmons, H.L., Jayne, S.R., 2002. Estimating tidally driven mixing in the deep ocean. Geophys. Res. Lett. 29, 2106.

St. Laurent, L.C., Simmons, H.L., 2006. Estimates of power consumed by mixing in the ocean interior. J. Clim. 19, 4877-4890.

St. Laurent, L., Naveira Garabato, A., Ledwell, J., Thurnherr, A., Toole, J., Watson, A. 2012. Turbulence and diapycnal mixing in Drake Passage. J. Phys. Oceanogr. 42 2143-2152.

Sun, S., Bleck, R., Rooth, C., Dukowicz, J., Chassignet, E., Killworth, P., 1999. Inclusion of thermobaricity in isopycnal-coordinate ocean models. J. Phys. Oceanogr. 29 2719-2729.

Tailleux, R., 2010. Entropy versus APE production: On the buoyancy power input in the oceans energy cycle. Geophys. Res. Lett. 37, L22603.

Taylor, G.I., 1919. Tidal friction in the Irish Sea. Philos. Trans. R. Soc. Lond., Ser. A 220, 1-33.

Thomas, Taylor, 2010. Reduction of usable wind-work on the general circulation by forced symmetric instability. Geophys. Res. Lett. 37, L18606. http://dx.doi.org 10.1029/2010GL044680.

Thoppil, P.G., Richman, J.G., Hogan, P.J., 2011. Energetics of a global ocean circulation model compared to observations. Geophys. Res. Lett. 38, L15607. http://dx.doi.org/10.1029/2011GL048347.

Treguier, A.M., 1992. Kinetic energy analysis of an eddy resolving, primitive equation model of the North Atlantic. J. Geophys. Res. 97, 687-701.

Trossman, D.S., Thompson, L., Hautala, S.L., 2011. Application of thin-plate splines in two-dimensions to oceanographic tracer data. J. Atmos. Ocean. Tech. 28 (11) $1522-1538$

von Storch, J.-S., Eden, C., Fast, I., Haak, H., Hernández-Deckers, D., Maier-Reimer, E. Marotzke, J., Stammer, D., 2012. An estimate of Lorenz energy cycle for the World Ocean based on the $1 / 10^{\circ}$ STORM/NCEP simulation. J. Phys. Oceanogr. 42, 2185-2205.

Wallcraft, A.J., Kara, A.B., Hurlburt, H.E., 2005. Convergence of Laplacian diffusion versus resolution of an ocean model. Geophys. Res. Lett. 32, L07604.

Wang, W., Huang, R.X., 2004. Wind energy input to the surface waves. J. Phys. Oceanogr. 34, 1276-1280.

Wang, Y., Qiao, F., Fang, G., Wei, Z., 2010. Application of wave-induced vertica mixing to the K-profile parameterization scheme. J. Geophys. Res.-Oceans 115, C09014, doi:10.1029/2009JC005856.

Watanabe, M., Hibiya, T., 1239. Global estimates of the wind-induced energy flux to inertial motions in the surface mixed layer. Geophys. Res. Lett. 29, 1239.

Waterman, S., Naveira Garabato, A.C., Polzin, K.L., 2013. Internal waves and turbulence in the Antarctic Circumpolar Current. J. Phys. Oceanogr. 43, 259282.

Webb, D.J., Suginohara, N., 2001. Vertical mixing in the ocean. Nature 409, 37.

Willebrand, W., Barnier, B., Boning, C., Dieterich, C., Killworth, P.D., Le Provost, C., Jia, Y., Molines, J.-M., New, A.L., 2001. Circulation characteristics in three eddypermitting models of the North Atlantic. Progr. Oceanogr. 48, 123-161.

Winters, K.B., Lombard, P.N., Riley, J.J., D'Asaro, E.A., 1995. Available potential energy and mixing in density-stratified fluids. J. Fluid Mech. 289, 115-128.

Wood, S.N., 2006. Generalized Additive Models: An Introduction with R. Chapman and Hall/CRC Press.

Wright, C.J., Scott, R.B., Arbic, B.K., Furnival, D.F., 2012. Bottom dissipation of subinertial currents at the Atlantic zonal boundaries. J. Geophys. Res.-Oceans 117, C03049.

Wright, C.J., Scott, R.B., Furnival, D., Ailliot, P. Vermet, F., 2013. Global observations of ocean-bottom subinertial current dissipation. J. Phys. Oceanogr. 43, 402-417.

Wunsch, C., 1998. The work done by the wind on the oceanic general circulation. J Phys. Oceanogr. 28, 2332-2340.

Wunsch, C., Ferrari, R., 2004. Vertical mixing, energy, and the general circulation of the oceans. Annu. Rev. Fluid. Mech. 36, 281-314.

Zhai, X., Johnson, H., Marshall, D., 2010. Significant sink of ocean-eddy energy near western boundaries. Nature Geosci. 3, 608-612.

Zhai, X., Johnson, H., Marshall, D., Wunsch, C., 2012. On the wind power input to the ocean general circulation. J. Phys. Oceanogr. 42, 1357-1365. 